\title{
Three-Component Radical Iodonitrosylative Cyclization of 1,6- Enynes under Metal-free Conditions
}

\author{
Shaoqun Zhu, ${ }^{*},{ }^{\dagger}$ Qi Cheng, ${ }^{\dagger}$ Haibo Yang, ${ }^{\dagger}$ Xiaoyun Chen, ${ }^{\star}$ Ying Han,${ }^{\dagger}$ Chaoguo \\ Yan, ${ }^{\dagger}$ Yaocheng Shi, ${ }^{\dagger}$ and Hong $\mathrm{Hou}^{\dagger}$ \\ 'School of Chemistry \& Chemical Engineering, Yangzhou University, Yangzhou, 225009, China \\ ¥School of Environmental and Chemical Engineering, Jiangsu University of Science and Technology, \\ Zhenjiang 212005, China \\ shaoqun.zhu@yzu.edu.cn
}

1. General information S2

2. General procedure for the preparation of starting materials

4. Characterizations of products 


\section{General information}

Unless otherwise noted, all commercially available compounds were used as provided without further purification. Solvents used in reactions were p.A. grade and dried only if indicated. Solvents for chromatography were technical grade and distilled prior to use. Analytical thin-layer chromatography (TLC) was performed on Merck silica gel aluminium plates with F-254 indicator, visualized by irradiation with UV light. Column chromatography was performed using silica gel Merck 60 (particle size 0.063-0.2 mm). Melting points were measured on a Yanaco Micro Melting Point Apparatus. ${ }^{1} \mathrm{H}$ NMR, ${ }^{13} \mathrm{C}\left\{{ }^{1} \mathrm{H}\right\}$ NMR and ${ }^{19} \mathrm{~F}\left\{{ }^{1} \mathrm{H}\right\}$ NMR were recorded on a Varian VNMR 400 or Bruker AV- 600 spectrometer in $\mathrm{CDCl}_{3}$. For ${ }^{1} \mathrm{H}$ NMR spectra, data are quoted in the following order: chemical shift $(\delta)$ in parts per million ( $\mathrm{ppm})$ downfield of tetramethylsilane, using residual protonated solvent as internal standard $\left(\mathrm{CDCl}_{3}\right.$ at $7.26 \mathrm{ppm}$, DMSO- $\mathrm{d}_{6}$ at $\left.2.50 \mathrm{ppm}\right)$. Multiplicities are indicated s (singlet), d (doublet), $\mathrm{t}$ (triplet), $\mathrm{m}$ (multiplet), dd (doublet of doublets), dt (doublet of triplets); coupling constants $(J)$ are in Hertz $(\mathrm{Hz})$. For proton-decoupled ${ }^{13} \mathrm{C}$ NMR spectra, chemical shifts $(J)$ are also quoted in parts per million (ppm) downfield of tetramethylsilane, using deuterated solvent as internal standard $\left(\mathrm{CDCl}_{3}\right.$ at $77.0 \mathrm{ppm}$, DMSO- $\mathrm{d}_{6}$ at $\left.39.5 \mathrm{ppm}\right)$. IR spectra were recorded on a Perkin Elmer Spectrum 100 FTIR (KBr disc) and are reported in terms of frequency of absorption $\left(\mathrm{cm}^{-1}\right)$. High resolution mass spectra (HRMS) were obtained on AB 5800 MALDITOF/TOF and are recorded using electrospray ionization (ESI). X-ray crystallographic data were collected using D8 quest X-ray diffractometer.

\section{General procedure for the preparation of starting materials}

Compounds 1a-1d, 1g-1y were prepared according to the previous reported procedures. ${ }^{[1-8]}$ Citations to the references containing characterization data for these compounds: $\mathbf{1 a}^{1}, \mathbf{1 b}^{1}, \mathbf{1 c}^{1}, \mathbf{1 d}^{2}$,

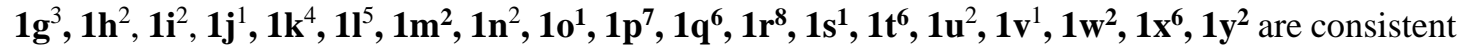
with the previous reports.

\section{The preparation of $1 \mathrm{e}$ and $1 \mathrm{f}$.}

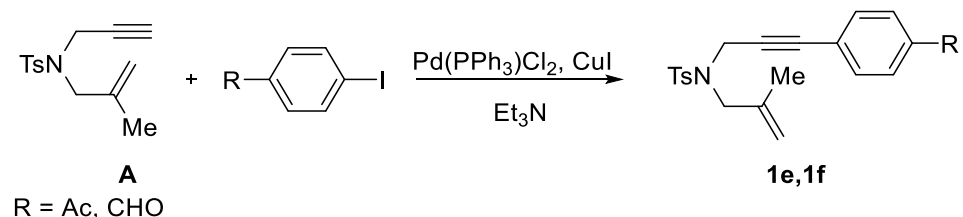

To a dried schlenk flask was added $\mathrm{Pd}\left(\mathrm{PPh}_{3}\right)_{2} \mathrm{Cl}_{2}(0.1 \mathrm{mmol}), \mathrm{CuI}(0.1 \mathrm{mmol})$, iodoarene $(5.5$ mmol), A (5.0 mmol, substrate $\mathbf{A}$ was prepared according to the previous reported procedures $\left.{ }^{[1]}\right)$ and freshly distilled $\mathrm{Et}_{3} \mathrm{~N}$ under argon. The resulting mixture was stirred at $50{ }^{\circ} \mathrm{C}$ overnight. And the reaction mixture was cooled to room temperature, filtered through diatomaceous earth, and washed with ethyl acetate. The filtrate was dried over anhydrous $\mathrm{Na}_{2} \mathrm{SO}_{4}$, and concentrated in vacuo. The crude mixture was purified by silica gel column chromatography to give $\mathbf{1 e}, \mathbf{1 f}$. 
N-(3-(4-formylphenyl)prop-2-yn-1-yl)-4-methyl-N-(2-methylallyl)benzenesulfonamide. (1e)

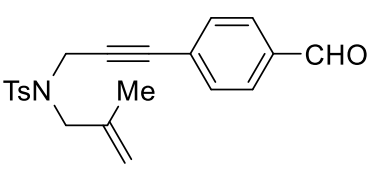

Yellow solid ( $1.67 \mathrm{~g}, 91 \%$ yield); m. p. $70-72^{\circ} \mathrm{C} ; \mathrm{R}_{f}=0.25$ (ethyl acetate/petroleum ether,1:9); ${ }^{1} \mathrm{H}$ NMR (400 MHz, $\left.\mathrm{CDCl}_{3}\right): \delta 9.96(\mathrm{~s}$, $1 \mathrm{H}), 7.74(\mathrm{t}, J=8.0 \mathrm{~Hz}, 4 \mathrm{H}), 7.23(\mathrm{~d}, J=8.0 \mathrm{~Hz}, 2 \mathrm{H}), 7.15(\mathrm{~d}, J=8.0$ $\mathrm{Hz}, 2 \mathrm{H}), 4.98$ (s, 2H), 4.26 (s, 2H), $3.78(\mathrm{~s}, 2 \mathrm{H}), 2.30$ (s, 3H), 1.79 (s, 3H). ${ }^{13} \mathrm{C}\left\{{ }^{1} \mathrm{H}\right\}$ NMR $(101$ $\left.\mathrm{MHz}, \mathrm{CDCl}_{3}\right): \delta 191.2,143.5,139.1,135.9,135.5,131.9,129.5,129.2,128.3,127.8,115.7,85.9$, 84.7, 52.9, 36.3, 21.4, 19.7. IR (neat): $v=3560,3002,1812,1432,1350,1202,1013,792,723,684$, 602, $490 \mathrm{~cm}^{-1}$; HRMS (ESI) m/z: [M+Na] $]^{+}$calcd for $\mathrm{C}_{21} \mathrm{H}_{21} \mathrm{NNaO}_{3} \mathrm{~S}^{+} 390.1134$; found 390.1137 .

N-(3-(4-acetylphenyl)prop-2-yn-1-yl)-4-methyl-N-(2-methylallyl) benzenesulfonamide. (1f)

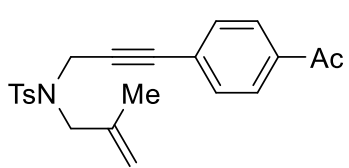

Yellow solid (1.72 g, 90\% yield); m. p. $49-51^{\circ} \mathrm{C} ; \mathrm{R}_{f}=0.25$ (ethyl acetate/petroleum ether,1:9); ${ }^{1} \mathrm{H}$ NMR $\left(400 \mathrm{MHz}, \mathrm{CDCl}_{3}\right): \delta 7.81(\mathrm{~d}, J=$ $8.0 \mathrm{~Hz}, 2 \mathrm{H}), 7.76(\mathrm{~d}, J=8.0 \mathrm{~Hz}, 2 \mathrm{H}), 7.24(\mathrm{~d}, J=8.0 \mathrm{~Hz}, 2 \mathrm{H}), 7.10$ (d, $J$ $=8.0 \mathrm{~Hz}, 2 \mathrm{H}), 4.99(\mathrm{~s}, 2 \mathrm{H}), 4.26(\mathrm{~s}, 2 \mathrm{H}), 3.79(\mathrm{~s}, 2 \mathrm{H}), 2.58(\mathrm{~s}, 3 \mathrm{H}), 2.32(\mathrm{~s}, 3 \mathrm{H}), 1.80(\mathrm{~s}, 3 \mathrm{H}) .{ }^{13} \mathrm{C}\left\{{ }^{1} \mathrm{H}\right\}$ NMR $\left(101 \mathrm{MHz}, \mathrm{CDCl}_{3}\right): \delta$ 197.2, 143.5, 139.1, 136.3, 135.9, 131.5, 129.5, 127.9, 127.8, 127.0, 115.7, 85.1, 84.8, 52.8, 36.3, 26.6, 21.4, 19.7. IR (neat): $v=3432,2984,1765,1451,1348,1163$, 1041, 822, 737, 665, 589, $550 \mathrm{~cm}^{-1}$; HRMS (ESI) m/z: [M+Na $]^{+}$calcd for $\mathrm{C}_{22} \mathrm{H}_{23} \mathrm{NNaO}_{3} \mathrm{~S}^{+} 404.1291$; found 404.1294 .

\section{General procedure for the preparation of product $4 a-4 y$}

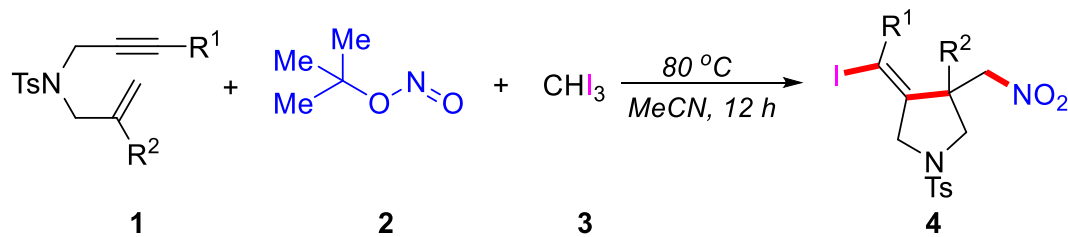

Enynes 1 (0.1 mmol, 1.0 equiv.), $\mathrm{CHI}_{3}$ (78.0 mg, 2.0 equiv.), and a stir bar were added to a sealed tube under a nitrogen atmosphere, $\mathrm{MeCN}(2 \mathrm{~mL})$ as solvent was then added. Then $2(0.18 \mathrm{mmol}$, 1.8 equiv.) was added, the mixture was stirred for $12 \mathrm{~h}$ at $80^{\circ} \mathrm{C}$ with an oil bath. The crude mixture was directly purified by flash column chromatography on silica gel (EtOAc/petroleum ether 1:15 to 1:3) to give the desired products $\mathbf{4 a - 4 y}$.

\section{Characterization of products}

(E)-4-(iodo(phenyl)methylene)-3-methyl-3-(nitromethyl)-1-tosylpyrrolidine (4a)

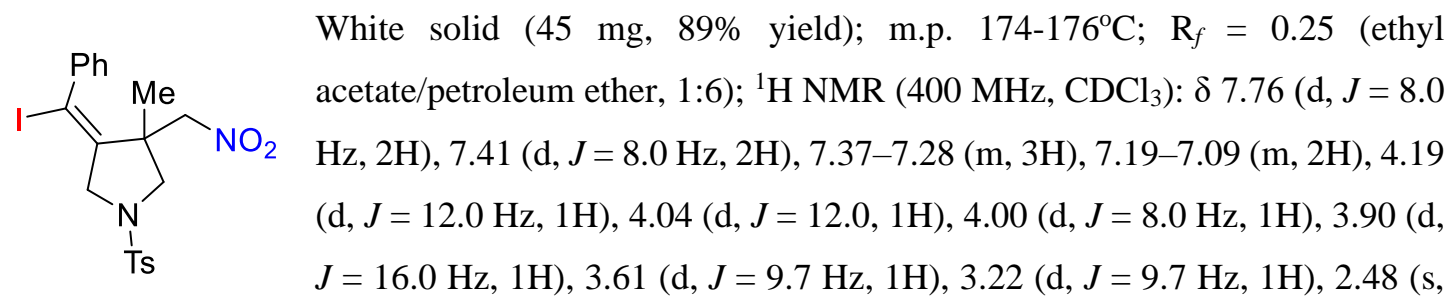

3H), 1.09 (s, 3H). ${ }^{13} \mathrm{C}\left\{{ }^{1} \mathrm{H}\right\}$ NMR (101 MHz, $\left.\mathrm{CDCl}_{3}\right): \delta 144.7,144.3,142.0,131.5,130.0,129.0$, 
128.7, 128.0, 127.5, 95.1, 79.7, 61.2, 59.2, 47.0, 22.5, 21.6. IR (neat): $v=3663,2926,1594,1445$, 1346, 1161, 1093, 1048, 908, 821, 710, 665, 586, $548 \mathrm{~cm}^{-1}$; HRMS (ESI) m/z: $[\mathrm{M}+\mathrm{H}]^{+}$calcd for $\mathrm{C}_{20} \mathrm{H}_{22} \mathrm{IN}_{2} \mathrm{O}_{4} \mathrm{~S}^{+}$513.0339; found 513.0346.

\section{(Z)-4-(iodo(phenyl)methylene)-3-methyl-3-(nitromethyl)tetrahydrofuran (4b)}<smiles>CC1(C[N+](=O)[O-])COC/C1=C(/I)c1ccccc1</smiles>

Colorless oil (18 mg, $50 \%$ yield); $\mathrm{R}_{f}=0.25$ (ethyl acetate/petroleum ether,1:20); ${ }^{1} \mathrm{H}$ NMR (400 MHz, $\mathrm{CDCl}_{3}$ ): $\delta 7.47-7.29$ (m, 3H), 7.27-7.20 (m, 2H), $4.46(\mathrm{~s}, 2 \mathrm{H}), 4.32(\mathrm{~d}, J=12.0 \mathrm{~Hz}, 1 \mathrm{H}), 4.28(\mathrm{~d}, J=8.0 \mathrm{~Hz}, 1 \mathrm{H}), 4.06(\mathrm{~d}$, $J=11.6 \mathrm{~Hz}, 1 \mathrm{H}), 3.80(\mathrm{~d}, J=9.3 \mathrm{~Hz}, 1 \mathrm{H}), 1.04(\mathrm{~s}, 3 \mathrm{H}) .{ }^{13} \mathrm{C}\left\{{ }^{1} \mathrm{H}\right\} \mathrm{NMR}(101$ $\mathrm{MHz}, \mathrm{CDCl}_{3}$ ): $\delta 148.0,142.1,128.9,128.7,127.7,91.6,80.4,80.0,79.5,48.5,20.9$. IR (neat): $v=$ 3663, 3297, 2381, 1717, 1547, 1408, 1265, 852, 756, $703 \mathrm{~cm}^{-1}$; HRMS (ESI) m/z: $[\mathrm{M}+\mathrm{Na}]^{+}$calcd for $\mathrm{C}_{13} \mathrm{H}_{14} \mathrm{INNaO}_{3}{ }^{+} 381.9911$; found 381.9921 .

dimethyl (E)-4-(iodomethylene)-3-methyl-3-(nitromethyl)cyclopentane-1,1-dicarboxylate (4c)<smiles>COC(=O)C1(C[N+](=O)[O-])C/C(=C(/I)c2ccccc2)C(C)(C[N+](=O)[O-])C1</smiles>
Colorless oil (33 mg, $70 \%$ yield); $\mathrm{R}_{f}=0.25$ (ethyl acetate/petroleum ether,1:3); ${ }^{1} \mathrm{H}$ NMR (400 MHz, $\mathrm{CDCl}_{3}$ ): $\delta$ 7.34-7.27 (m, 3H), 7.17 (d, $J=$ $8.0 \mathrm{~Hz}, 2 \mathrm{H}), 4.15(\mathrm{~d}, J=12.0 \mathrm{~Hz}, 1 \mathrm{H}), 3.93(\mathrm{~d}, J=12.0 \mathrm{~Hz}, 1 \mathrm{H}), 3.77(\mathrm{~s}$, $6 \mathrm{H}), 3.42(\mathrm{~d}, J=20.0 \mathrm{~Hz}, 1 \mathrm{H}), 3.30(\mathrm{~d}, J=16.0 \mathrm{~Hz}, 1 \mathrm{H}), 2.88$ (d, $J=16.0$ $\mathrm{Hz}, 1 \mathrm{H}), 2.54(\mathrm{~d}, J=16.0 \mathrm{~Hz}, 1 \mathrm{H}), 1.05(\mathrm{~s}, 3 \mathrm{H}) .{ }^{13} \mathrm{C}\left\{{ }^{1} \mathrm{H}\right\} \mathrm{NMR}(101 \mathrm{MHz}$, $\left.\mathrm{CDCl}_{3}\right): \delta 171.51,171.46,148.7,143.0,131.6,128.6,127.9,95.5,81.9,56.2,53.3,53.1,50.6,46.4$, 46.3, 25.6. IR (neat): $v=2954,1736,1551,1437,1377,1265,1207,1071,959,910,857,706 \mathrm{~cm}^{-1}$; HRMS (ESI) m/z: $[\mathrm{M}+\mathrm{H}]^{+}$calcd for $\mathrm{C}_{18} \mathrm{H}_{21} \mathrm{INO}_{6}{ }^{+} 474.0408$; $[\mathrm{M}+\mathrm{H}]^{+}$calcd for $\mathrm{C}_{18} \mathrm{H}_{21} \mathrm{INO}_{6}{ }^{+}$ 474.0408; found 474.0412.

(Z)-4-(iodo(phenyl)methylene)-3-methyl-3-(nitromethyl)-1-tosylpyrrolidin-2-one (4d)

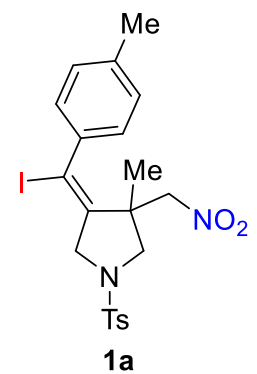

White solid. (47.8 mg, 91\% yield), m. p. $170-171{ }^{\circ} \mathrm{C} ; \mathrm{R}_{f}=0.25$ (ethyl acetate/petroleum ether, 1:6); ${ }^{1} \mathrm{H} \mathrm{NMR}\left(400 \mathrm{MHz}, \mathrm{CDCl}_{3}\right): \delta 7.75$ (d, $J=8.0$ $\mathrm{Hz}, 2 \mathrm{H}), 7.40(\mathrm{~d}, J=8.0 \mathrm{~Hz}, 2 \mathrm{H}), 7.14(\mathrm{~d}, J=8.0 \mathrm{~Hz}, 2 \mathrm{H}), 7.01(\mathrm{~d}, J=8.0 \mathrm{~Hz}$, $2 \mathrm{H}), 4.20(\mathrm{~d}, J=12.0 \mathrm{~Hz}, 1 \mathrm{H}), 4.03-3.99(\mathrm{~m}, 2 \mathrm{H}), 3.87(\mathrm{~d}, J=16.0 \mathrm{~Hz}, 1 \mathrm{H})$, $3.59(\mathrm{~d}, J=8.0 \mathrm{~Hz}, 1 \mathrm{H}), 3.22(\mathrm{~d}, J=8.0 \mathrm{~Hz}, 1 \mathrm{H}), 2.48(\mathrm{~s}, 3 \mathrm{H}), 2.34(\mathrm{~s}, 3 \mathrm{H})$, 1.11 (s, 3H). ${ }^{13} \mathrm{C}\left\{{ }^{1} \mathrm{H}\right\}$ NMR (101 MHz, $\left.\mathrm{CDCl}_{3}\right): \delta 144.5,144.3,139.18,139.17$, $131.5,129.9,129.4,128.0,127.4,95.6,79.7,61.2,59.2,46.9,22.5,21.6,21.3$. IR (neat): $v=3648$, 2926, 1657, 1598, 1456, 1347, 1163, 1091, 1047, 820, 719, 665, 591, $548 \mathrm{~cm}^{-1}$; HRMS (ESI) m/z: $[\mathrm{M}+\mathrm{Na}]^{+}$calcd for $\mathrm{C}_{21} \mathrm{H}_{23} \mathrm{IN}_{2} \mathrm{NaO}_{4} \mathrm{~S}^{+}$549.0315; found 549.0317.

(Z)-4-(iodo(4-methyl-4-(nitromethyl)-1-tosylpyrrolidin-3-ylidene)methyl) benzaldehyde (4e) 


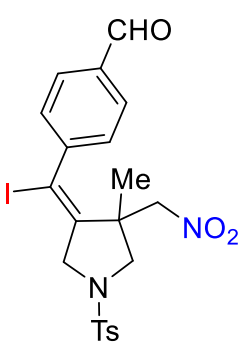

White solid (31 mg, $58 \%$ yield); m. p. $157-159^{\circ} \mathrm{C} ; \mathrm{R}_{f}=0.25$ (ethyl acetate/petroleum ether, 1:3); ${ }^{1} \mathrm{H}$ NMR (400 MHz, $\left.\mathrm{CDCl}_{3}\right): \delta 10.00$ (s, $1 \mathrm{H}$ ), $7.87(\mathrm{~d}, J=8.0 \mathrm{~Hz}, 2 \mathrm{H}), 7.74(\mathrm{t}, J=7.9 \mathrm{~Hz}, 2 \mathrm{H}), 7.41(\mathrm{~d}, J=8.0 \mathrm{~Hz}, 2 \mathrm{H}), 7.32$ $(\mathrm{d}, J=8.0 \mathrm{~Hz}, 2 \mathrm{H}), 4.23(\mathrm{~d}, J=12.0 \mathrm{~Hz}, 1 \mathrm{H}), 4.07(\mathrm{~d}, J=12.0 \mathrm{~Hz}, 1 \mathrm{H}), 4.01$ $(\mathrm{d}, J=12.0 \mathrm{~Hz}, 1 \mathrm{H}), 3.92(\mathrm{~d}, J=16.0 \mathrm{~Hz}, 1 \mathrm{H}), 3.67(\mathrm{~d}, J=12.0 \mathrm{~Hz}, 1 \mathrm{H}), 3.18$ $(\mathrm{d}, J=8.0 \mathrm{~Hz}, 1 \mathrm{H}), 2.48(\mathrm{~s}, 3 \mathrm{H}), 1.05(\mathrm{~s}, 3 \mathrm{H}) \cdot{ }^{13} \mathrm{C}\left\{{ }^{1} \mathrm{H}\right\} \mathrm{NMR}(101 \mathrm{MHz}$, $\left.\mathrm{CDCl}_{3}\right): \delta 190.9,147.6,145.6,144.5,136.1,131.5,130.0,129.9,128.4,128.0,92.979 .7,61.3,59.1$, 47.3 22.4, 21.6. IR (neat): $v=3649,3303,2380,1699,1596,1553,1344,1205,905,822,745,663$ $\mathrm{cm}^{-1}$; HRMS (ESI) m/z: [M+Na] $]^{+}$calcd for $\mathrm{C}_{21} \mathrm{H}_{21} \mathrm{IN}_{2} \mathrm{NaO}_{5} \mathrm{~S}^{+}$563.0108; found 563.0102.

(Z)-1-(4-(iodo(4-methyl-4-(nitromethyl)-1-tosylpyrrolidin-3-ylidene)methyl) phenyl)ethan-1one (4f)

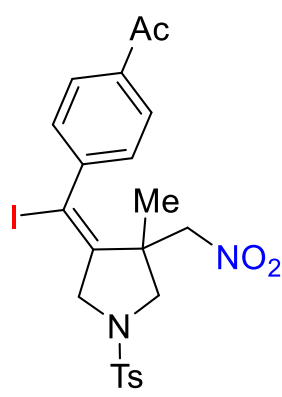

Yellow gum (33.4 mg, 60\% yield); $\mathrm{R}_{f}=0.25$ (ethyl acetate/petroleum ether,
1:6); ${ }^{1} \mathrm{H} \mathrm{NMR}\left(400 \mathrm{MHz}, \mathrm{CDCl}_{3}\right): \delta 7.93(\mathrm{~d}, J=12.0 \mathrm{~Hz}, 2 \mathrm{H}), 7.75(\mathrm{~d}, J=$
$8.0 \mathrm{~Hz}, 2 \mathrm{H}), 7.40(\mathrm{~d}, J=8.0 \mathrm{~Hz}, 2 \mathrm{H}), 7.25(\mathrm{~d}, J=8.0 \mathrm{~Hz}, 2 \mathrm{H}), 4.21(\mathrm{~d}, J=$
$12.0 \mathrm{~Hz}, 1 \mathrm{H}), 4.05(\mathrm{~d}, J=12.0 \mathrm{~Hz}, 1 \mathrm{H}), 4.01(\mathrm{~d}, J=12.0 \mathrm{~Hz}, 1 \mathrm{H}), 3.94(\mathrm{~d}, J$
$=12.0 \mathrm{~Hz}, 1 \mathrm{H}), 3.65(\mathrm{~d}, J=8.0 \mathrm{~Hz}, 1 \mathrm{H}), 3.19(\mathrm{~d}, J=8.0 \mathrm{~Hz}, 1 \mathrm{H}), 2.60(\mathrm{~s}$,
$3 \mathrm{H}), 2.47(\mathrm{~s}, 3 \mathrm{H}), 1.06(\mathrm{~s}, 3 \mathrm{H}) .{ }^{13} \mathrm{C}\left\{{ }^{1} \mathrm{H}\right\} \mathrm{NMR}(101 \mathrm{MHz}, \mathrm{CDCl}): \delta 196.9$,
$146.4,145.4,144.5,137.0,131.6,130.0,128.7,128.0,127.9,93.3,79.6,61.2$, 59.2, 47.2, 26.6, 22.4, 21.7. IR (neat): $v=3648,3299,2383,1691,1549,1433,1343,1158,1089$, 812, 766, $662 \mathrm{~cm}^{-1}$; HRMS (ESI) m/z: $[\mathrm{M}+\mathrm{Na}]^{+}$calcd for $\mathrm{C}_{22} \mathrm{H}_{23} \mathrm{IN}_{2} \mathrm{NaO}_{5} \mathrm{~S}^{+}$577.0265; found 577.0252

\section{(Z)-4-(iodo(4-(trifluoromethyl)phenyl)methylene)-3-methyl-3-(nitromethyl)-1-}

tosylpyrrolidine (4g)

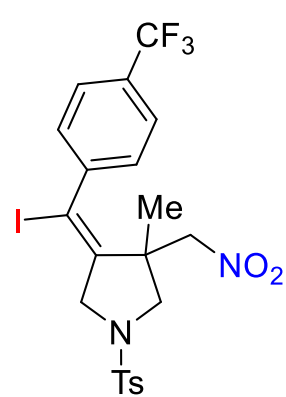

White solid (42 mg,72\% yield); m. p. $158-160^{\circ} \mathrm{C} ; \mathrm{R}_{f}=0.25$ (ethyl acetate/petroleum ether, $1: 6) ;{ }^{1} \mathrm{H}$ NMR $\left(400 \mathrm{MHz}, \mathrm{CDCl}_{3}\right): \delta 7.76$ (d, $J=8.0$ $\mathrm{Hz}, 2 \mathrm{H}), 7.62(\mathrm{~d}, J=8.0 \mathrm{~Hz}, 2 \mathrm{H}), 7.41(\mathrm{~d}, J=8.0 \mathrm{~Hz}, 2 \mathrm{H}), 7.28(\mathrm{~d}, J=8.0$ $\mathrm{Hz}, 2 \mathrm{H}), 4.22(\mathrm{~d}, J=12.0, \mathrm{~Hz}, 1 \mathrm{H}), 4.06(\mathrm{~d}, J=12.0, \mathrm{~Hz}, 1 \mathrm{H}), 4.01$ (d, $J=$ $16.0 \mathrm{~Hz}, 1 \mathrm{H}), 3.95(\mathrm{~d}, J=12.0 \mathrm{~Hz}, 1 \mathrm{H}), 3.66(\mathrm{~d}, J=8.0 \mathrm{~Hz}, 1 \mathrm{H}), 3.19(\mathrm{~d}, J=$ $8.0 \mathrm{~Hz}, 1 \mathrm{H}), 2.48$ (s, 3H), 1.06 (s, 3H). ${ }^{13} \mathrm{C}\left\{{ }^{1} \mathrm{H}\right\} \mathrm{NMR}\left(101 \mathrm{MHz}, \mathrm{CDCl}_{3}\right): \delta$ $145.8,145.5,144.5,131.5,131.3(\mathrm{q}, J=25.3 \mathrm{~Hz}, 1 \mathrm{C}), 130.0,128.1,128.0$, $126.8(\mathrm{q}, J=274.7 \mathrm{~Hz}, 1 \mathrm{C}), 125.6(\mathrm{~d}, J=3.0 \mathrm{~Hz}, 1 \mathrm{C}), 92.7,79.7,61.3,59.2$, 47.3, 22.5, 21.6. ${ }^{19} \mathrm{~F}\left\{{ }^{1} \mathrm{H}\right\}$ NMR ( $\left.376 \mathrm{MHz}, \mathrm{CDCl}_{3}\right) \delta-62.8$ (s, 3F). IR (neat): $v=3625,3302,2381$, 1667, 1554, 1326, 1261, 1165, 1126, 1059, 839, $754 \mathrm{~cm}^{-1}$; HRMS (ESI) m/z: $[\mathrm{M}+\mathrm{Na}]^{+}$calcd for $\mathrm{C}_{21} \mathrm{H}_{20} \mathrm{~F}_{3} \mathrm{IN}_{2} \mathrm{NaO}_{4} \mathrm{~S}^{+}$603.0033; found 603.0034.

(Z)-4-((4-fluorophenyl)iodomethylene)-3-methyl-3-(nitromethyl)-1-tosylpyrrolidine (4h) 


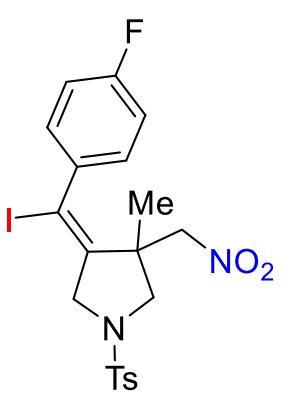

White solid (31.8 mg, $60 \%$ yield); m. p. $80-82^{\circ} \mathrm{C} ; \mathrm{R}_{f}=0.25$ (ethyl acetate/petroleum ether, $1: 6) ;{ }^{1} \mathrm{H}$ NMR $\left(400 \mathrm{MHz}, \mathrm{CDCl}_{3}\right): \delta 7.75$ (d, $J=8.0$ $\mathrm{Hz}, 2 \mathrm{H}), 7.40(\mathrm{~d}, J=8.0 \mathrm{~Hz}, 2 \mathrm{H}), 7.17-7.11(\mathrm{~m}, 2 \mathrm{H}), 7.04(\mathrm{t}, J=8.0 \mathrm{~Hz}$, 2H), $4.20(\mathrm{~d}, J=12.0 \mathrm{~Hz}, 1 \mathrm{H}), 4.03(\mathrm{~d}, J=12.0 \mathrm{~Hz}, 1 \mathrm{H}), 3.99(\mathrm{~d}, J=16.0 \mathrm{~Hz}$, $1 \mathrm{H}), 3.89(\mathrm{~d}, J=16.0 \mathrm{~Hz}, 1 \mathrm{H}), 3.63(\mathrm{~d}, J=8.0 \mathrm{~Hz}, 1 \mathrm{H}), 3.20(\mathrm{~d}, J=8.0 \mathrm{~Hz}$, 1H), 2.47 (s, 3H), 1.08 (s, 3H). ${ }^{13} \mathrm{C}\left\{{ }^{1} \mathrm{H}\right\} \operatorname{NMR}\left(101 \mathrm{MHz}, \mathrm{CDCl}_{3}\right): \delta 163.6$, $161.1,145.5,144.4,138.1$ (d, $J=4.0 \mathrm{~Hz}, 1 \mathrm{C}), 130.0,129.5$ (d, $J=8.0 \mathrm{~Hz}$, 1C), 128.0, $115.9(\mathrm{~d}, J=22.0 \mathrm{~Hz}, 1 \mathrm{C}), 93.9,79.6,61.2,59.2,47.0,22.5,21.6 .{ }^{19} \mathrm{~F}\left\{{ }^{1} \mathrm{H}\right\}$ NMR $(376$ $\mathrm{MHz}, \mathrm{CDCl}_{3}$ ) $\delta-110.6$ (s, 1F). IR (neat): $v=3648,3300,2382,1705,1546,1408,1223,1157,831$, $664 \mathrm{~cm}^{-1}$; HRMS (ESI) m/z: [M+Na] $]^{+}$calcd for $\mathrm{C}_{20} \mathrm{H}_{20} \mathrm{FIN}_{2} \mathrm{NaO}_{4} \mathrm{~S}^{+} 553.0065$; found 553.0066.

\section{(Z)-4-((4-chlorophenyl)iodomethylene)-3-methyl-3-(nitromethyl)-1-tosylpyrrolidine (4i)}

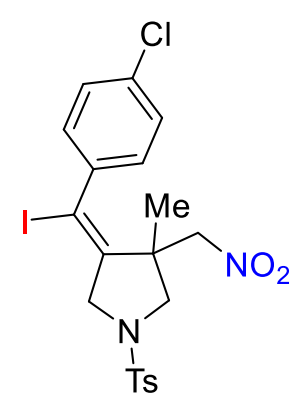

White solid (53.5 mg, 98\% yield); m. p. $208-210^{\circ} \mathrm{C} ; \mathrm{R}_{f}=0.25$ (ethyl acetate/petroleum ether, 1:6); ${ }^{1} \mathrm{H}$ NMR $\left(400 \mathrm{MHz}, \mathrm{CDCl}_{3}\right): \delta 7.75(\mathrm{~d}, J=8.0$ $\mathrm{Hz}, 2 \mathrm{H}), 7.40(\mathrm{~d}, J=8.0 \mathrm{~Hz}, 2 \mathrm{H}), 7.32(\mathrm{~d}, J=8.0 \mathrm{~Hz}, 2 \mathrm{H}), 7.09$ (d, $J=8.0$ $\mathrm{Hz}, 2 \mathrm{H}), 4.21$ (d, $J=12.0 \mathrm{~Hz}, 1 \mathrm{H}), 4.05(\mathrm{~d}, J=12.0 \mathrm{~Hz}, 1 \mathrm{H}), 3.99$ (d, $J=16.0$ $\mathrm{Hz}, 1 \mathrm{H}), 3.90(\mathrm{~d}, J=16.0 \mathrm{~Hz}, 1 \mathrm{H}), 3.63(\mathrm{~d}, J=8.0 \mathrm{~Hz}, 1 \mathrm{H}), 3.20(\mathrm{~d}, J=12.0$ $\mathrm{Hz}, 1 \mathrm{H}), 2.47$ (s, 3H), $1.08(\mathrm{~s}, 3 \mathrm{H}) .{ }^{13} \mathrm{C}\left\{{ }^{1} \mathrm{H}\right\}$ NMR $\left(101 \mathrm{MHz}, \mathrm{CDCl}_{3}\right): \delta 145.5$, 144.4, 140.4, 135.0, 131.5, 130.0, 129.0, 128.9, 128.0, 93.5, 79.7, 61.3, 59.2,

47.1, 22.5, 21.6. IR (neat): $v=3638,2981,1592,1552,1347,1162,1092,1049,1009,826,735$, $665,591,541 \mathrm{~cm}^{-1}$; HRMS (ESI) m/z: [M+Na] $]^{+}$calcd for $\mathrm{C}_{20} \mathrm{H}_{20} \mathrm{ClIN}_{2} \mathrm{NaO}_{4} \mathrm{~S}^{+}$568.9769; found 568.9767.

\section{(Z)-4-((4-bromophenyl)iodomethylene)-3-methyl-3-(nitromethyl)-1-tosylpyrrolidine (4j)}

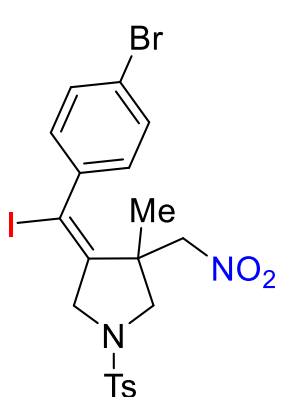

White solid (50.2 mg, 85\% yield); $\mathrm{R}_{f}=0.25$ (ethyl acetate/petroleum ether, 1:6); ${ }^{1} \mathrm{H}$ NMR (400 MHz, $\left.\mathrm{CDCl}_{3}\right): \delta 7.75(\mathrm{~d}, J=8.0 \mathrm{~Hz}, 2 \mathrm{H}), 7.48(\mathrm{~d}, J=$ $8.0 \mathrm{~Hz}, 2 \mathrm{H}), 7.40(\mathrm{~d}, J=8.0 \mathrm{~Hz}, 2 \mathrm{H}), 7.03(\mathrm{~d}, J=8.0 \mathrm{~Hz}, 2 \mathrm{H}), 4.21(\mathrm{~d}, J=$ $12.0 \mathrm{~Hz}, 1 \mathrm{H}), 4.06(\mathrm{~d}, J=12.0 \mathrm{~Hz}, 1 \mathrm{H}), 4.00(\mathrm{~d}, J=16.0 \mathrm{~Hz}, 1 \mathrm{H}), 3.90(\mathrm{~d}$, $J=16.0 \mathrm{~Hz}, 1 \mathrm{H}), 3.63(\mathrm{~d}, J=8.0 \mathrm{~Hz}, 1 \mathrm{H}), 3.20(\mathrm{~d}, J=12.0 \mathrm{~Hz}, 1 \mathrm{H}), 2.48$ (s, 3H), 1.08 (s, 3H). ${ }^{13} \mathrm{C}\left\{{ }^{1} \mathrm{H}\right\}$ NMR (101 MHz, $\left.\mathrm{CDCl}_{3}\right): \delta$ 145.5, 144.4, 140.9, 132.0, 131.6, 130.0, 129.2, 128.0, 123.2, 93.4, 79.7, 61.3, 59.2, 47.1, 22.6, 21.6. IR (neat): $v=3645,2973,2309,1712,1550,1384,1343,1159,1005,816,669,487 \mathrm{~cm}^{-}$ 1; HRMS (ESI) m/z: [M+Na] $]^{+}$calcd for $\mathrm{C}_{20} \mathrm{H}_{20} \mathrm{BrIN}_{2} \mathrm{NaO}_{4} \mathrm{~S}^{+}$612.9264; found 612.9241 .

methyl (Z)-4-(iodo(4-methyl-4-(nitromethyl)-1-tosylpyrrolidin-3-ylidene)methyl) benzoate $(4 k)$ 


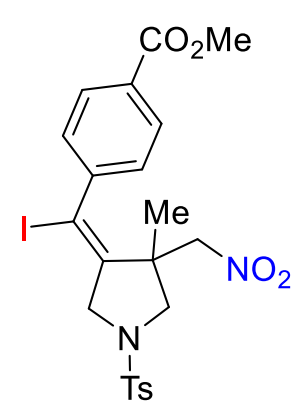

White solid (39.9 mg, 70\% yield); m. p. 206-208 ${ }^{\circ}$; $\mathrm{R}_{f}=0.25$ (ethyl acetate/ petroleum ether, 1:6); ${ }^{1} \mathrm{H} \mathrm{NMR}\left(400 \mathrm{MHz}, \mathrm{CDCl}_{3}\right): \delta 8.01(\mathrm{~d}, J=8.0 \mathrm{~Hz}, 2 \mathrm{H})$, $7.75(\mathrm{~d}, J=8.0 \mathrm{~Hz}, 2 \mathrm{H}), 7.41(\mathrm{~d}, J=8.0 \mathrm{~Hz}, 2 \mathrm{H}), 7.23(\mathrm{~d}, J=8.0 \mathrm{~Hz}, 2 \mathrm{H})$, $4.20(\mathrm{~d}, J=12.0 \mathrm{~Hz}, 1 \mathrm{H}), 4.02(\mathrm{~d}, J=12.0 \mathrm{~Hz}, 1 \mathrm{H}), 4.01(\mathrm{~d}, J=16.0 \mathrm{~Hz}, 1 \mathrm{H})$, $3.93(\mathrm{~d}, J=16.0 \mathrm{~Hz}, 1 \mathrm{H}), 3.92(\mathrm{~s}, 3 \mathrm{H}), 3.64(\mathrm{~d}, J=8.0 \mathrm{~Hz}, 1 \mathrm{H}), 3.19$ (d, $J=$ $8.0 \mathrm{~Hz}, 1 \mathrm{H}), 2.48(\mathrm{~s}, 3 \mathrm{H}), 1.06(\mathrm{~s}, 3 \mathrm{H}) .{ }^{13} \mathrm{C}\left\{{ }^{1} \mathrm{H}\right\} \mathrm{NMR}\left(101 \mathrm{MHz}, \mathrm{CDCl}_{3}\right): \delta$ 165.9, 146.3, 145.4, 144.4, 131.5, 130.5, 130.0, 129.9, 128.0, 127.7, 93.9, 79.6, 61.2, 59.2, 52.4, 47.2, 22.4, 21.6. IR (neat): $v=3650,3289,2962,2370,1370,1430,1344,1265$, 1157, 1034, 926, 754, $667 \mathrm{~cm}^{-1}$; HRMS (ESI) m/z: $[\mathrm{M}+\mathrm{Na}]^{+}$calcd for $\mathrm{C}_{22} \mathrm{H}_{23} \mathrm{IN}_{2} \mathrm{NaO}_{6} \mathrm{~S}^{+} 593.0214$; found 593.0207.

(Z)-4-(iodo(3-(trifluoromethyl)phenyl)methylene)-3-methyl-3-(nitromethyl)-1tosylpyrrolidine (41)

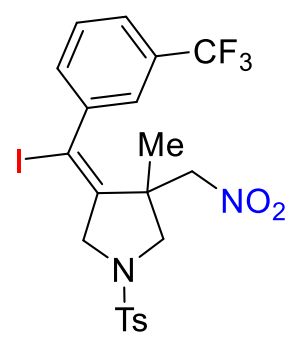

Yellow gum (40 mg, 68\% yield); $\mathrm{R}_{f}=0.25$ (ethyl acetate/petroleum ether,1:6); ${ }^{1} \mathrm{H}$ NMR (400 MHz, $\left.\mathrm{CDCl}_{3}\right): \delta 7.76(\mathrm{~d}, J=8.0 \mathrm{~Hz}, 2 \mathrm{H}), 7.58(\mathrm{~d}$, $J=8.0 \mathrm{~Hz}, 1 \mathrm{H}), 7.50(\mathrm{t}, J=8.0 \mathrm{~Hz}, 1 \mathrm{H}), 7.41(\mathrm{~d}, J=8.0 \mathrm{~Hz}, 3 \mathrm{H}), 7.35(\mathrm{~d}, J$ $=8.0 \mathrm{~Hz}, 1 \mathrm{H}), 4.19(\mathrm{~d}, J=12.0 \mathrm{~Hz}, 1 \mathrm{H}), 4.03(\mathrm{~d}, J=12.0 \mathrm{~Hz}, 1 \mathrm{H}), 3.97(\mathrm{~d}$, $J=4.0 \mathrm{~Hz}, 2 \mathrm{H}), 3.67(\mathrm{~d}, J=8.0 \mathrm{~Hz}, 1 \mathrm{H}), 3.18(\mathrm{~d}, J=8.0 \mathrm{~Hz}, 1 \mathrm{H}), 2.48(\mathrm{~s}$, $3 \mathrm{H}), 1.03(\mathrm{~s}, 3 \mathrm{H}) .{ }^{13} \mathrm{C}\left\{{ }^{1} \mathrm{H}\right\} \mathrm{NMR}\left(101 \mathrm{MHz}, \mathrm{CDCl}_{3}\right): \delta 146.1,144.5,142.7$, $131.3,130.8,130.0,129.4,127.2,125.7(\mathrm{~d}, J=4.0 \mathrm{~Hz}, 1 \mathrm{C}), 123.1(\mathrm{q}, J=273.7 \mathrm{~Hz}, 1 \mathrm{C}), 122.0,119.3$, 92.6, 79.6, 61.3, 59.2, 47.2, 22.4, 21.6. ${ }^{19} \mathrm{~F}\left\{{ }^{1} \mathrm{H}\right\}$ NMR $\left(376 \mathrm{MHz}, \mathrm{CDCl}_{3}\right) \delta-62.9$ (s, 3F). IR (neat): $v=3647,3297,2378,1716,1553,1432,1333,1265,1165,1129,819,698 \mathrm{~cm}^{-1}$; HRMS (ESI) m/z: $[\mathrm{M}+\mathrm{Na}]^{+}$calcd for $\mathrm{C}_{21} \mathrm{H}_{20} \mathrm{~F}_{3} \mathrm{IN}_{2} \mathrm{NaO}_{4} \mathrm{~S}^{+}$603.0033; found 603.0026 .

\section{(Z)-4-(iodo(3-methoxyphenyl)methylene)-3-methyl-3-(nitromethyl)-1-tosylpyrrolidine (4m)}

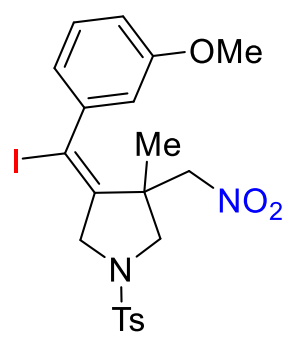

Yellow gum (46.6 mg, 86\% yield); $\mathrm{R}_{f}=0.25$ (ethyl acetate/petroleum ether, 1:6); ${ }^{1} \mathrm{H}$ NMR (400 MHz, $\left.\mathrm{CDCl}_{3}\right): \delta 7.75(\mathrm{~d}, J=8.0 \mathrm{~Hz}, 2 \mathrm{H}), 7.40(\mathrm{~d}, J=$ $8.0 \mathrm{~Hz}, 2 \mathrm{H}), 7.24(\mathrm{~d}, J=8.0 \mathrm{~Hz}, 1 \mathrm{H}), 6.87-6.81(\mathrm{~m}, 1 \mathrm{H}), 6.72(\mathrm{~d}, J=8.0$ $\mathrm{Hz}, 1 \mathrm{H}), 6.65$ (s, 1H), $4.20(\mathrm{~d}, J=12.0 \mathrm{~Hz}, 1 \mathrm{H}), 4.06(\mathrm{~d}, J=12.0 \mathrm{~Hz}, 1 \mathrm{H})$, $4.00(\mathrm{~s}, 1 \mathrm{H}), 3.87(\mathrm{~d}, J=16.0 \mathrm{~Hz}, 1 \mathrm{H}), 3.78(\mathrm{~s}, 3 \mathrm{H}), 3.59(\mathrm{~d}, J=8.0 \mathrm{~Hz}, 1 \mathrm{H})$, $3.23(\mathrm{~d}, J=8.0 \mathrm{~Hz}, 1 \mathrm{H}), 2.47(\mathrm{~s}, 3 \mathrm{H}), 1.13(\mathrm{~s}, 3 \mathrm{H}) .{ }^{13} \mathrm{C}\left\{{ }^{1} \mathrm{H}\right\} \mathrm{NMR}(101 \mathrm{MHz}$, $\left.\mathrm{CDCl}_{3}\right): \delta 159.3,144.6,144.3,143.1,131.6,129.9,129.8,128.0,119.7,114.6,113.2,94.7,79.6$, 61.2, 59.3, 55.3, 47.0, 22.6, 21.6. IR (neat): $v=3666,2934,2381,1589,1552,1469,1346,1259$, 1161, 1092, 1044, 822, 734, 664, 587, $548 \mathrm{~cm}^{-1}$; HRMS (ESI) m/z: $[\mathrm{M}+\mathrm{Na}]^{+}$calcd for $\mathrm{C}_{21} \mathrm{H}_{23} \mathrm{IN}_{2} \mathrm{NaO}_{5} \mathrm{~S}^{+}$565.0265; found 565.0250.

(Z)-4-(iodo(2-methoxyphenyl)methylene)-3-methyl-3-(nitromethyl)-1-tosylpyrrolidine (4n) 


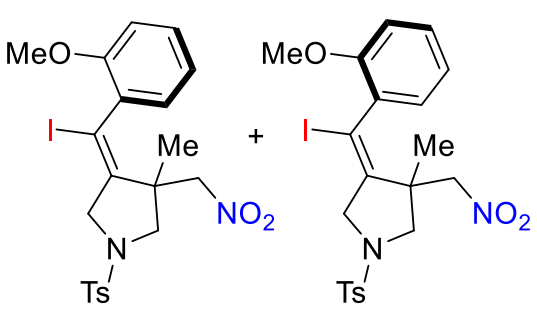

Yellow gum (22 mg, $40 \%$ yield, $\mathrm{dr}=2: 3$ or $3: 2) ; \mathrm{R}_{f}=0.25$ (ethyl acetate/ petroleum ether, 1:3); ${ }^{1} \mathrm{H}$ NMR $(400 \mathrm{MHz}$, $\left.\mathrm{CDCl}_{3}\right): \delta 7.77(\mathrm{~d}, J=8.0 \mathrm{~Hz}, 2 \mathrm{H}), 7.41(\mathrm{~d}, J=4.0 \mathrm{~Hz}, 2 \mathrm{H})$, $7.32(\mathrm{t}, J=8.0 \mathrm{~Hz}, 1 \mathrm{H}), 7.07-6.98(\mathrm{~m}, 1 \mathrm{H}), 6.95-6.86(\mathrm{~m}$, 2H), $4.31(\mathrm{~d}, J=12.0 \mathrm{~Hz}, 1 \mathrm{H}), 4.04(\mathrm{~d}, J=12.0 \mathrm{~Hz}, 1 \mathrm{H})$, $3.98(\mathrm{~d}, J=4.0 \mathrm{~Hz}, 1 \mathrm{H}), 3.93(\mathrm{~d}, J=12.0 \mathrm{~Hz}, 1 \mathrm{H}), 3.86(\mathrm{~s}$, 3H), $3.74(\mathrm{~d}, J=28.0 \mathrm{~Hz}, 1 \mathrm{H}), 3.15$ (d, $J=36.0,12.0 \mathrm{~Hz}, 1 \mathrm{H}), 2.48$ (s, 3H), 1.09 (s, 2H), 0.93 (s, 1H). ${ }^{13} \mathrm{C}\left\{{ }^{1} \mathrm{H}\right\}$ NMR $\left(100 \mathrm{MHz}, \mathrm{CDCl}_{3}\right): \delta 155.0,145.1,144.2,130.9,130.0,129.7,128.7,128.1$, 120.6, 111.7, 91.6, 80.0, 78.4, 61.0, 59.2, 55.5, 47.0, 21.6, 19.4. IR (neat): $v=3664,2934,2845$, 2230, 1725, 1592, 1553, 1459, 1252, 1163, 1098, $666 \mathrm{~cm}^{-1}$; HRMS (ESI) m/z: [M+Na] ${ }^{+}$calcd for $\mathrm{C}_{21} \mathrm{H}_{23} \mathrm{IN}_{2} \mathrm{NaO}_{5} \mathrm{~S}^{+}$565.0265; found 565.0257.

\section{(Z)-2-(iodo(4-methyl-4-(nitromethyl)-1-tosylpyrrolidin-3-ylidene)methyl) pyridine (4o)}

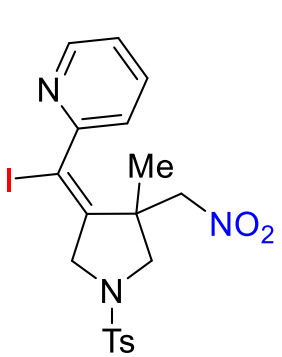

White solid (32 mg, 62\% yield); $\mathrm{R}_{f}=0.25$ (ethyl acetate/petroleum ether, $1: 3$ ); ${ }^{1} \mathrm{H}$ NMR (400 MHz, CDCl 3$): \delta 8.53(\mathrm{~d}, J=4.0 \mathrm{~Hz}, 1 \mathrm{H}), 8.44(\mathrm{~s}, 1 \mathrm{H}), 7.75(\mathrm{~d}$, $J=8.0 \mathrm{~Hz}, 2 \mathrm{H}), 7.49(\mathrm{~d}, J=8.0 \mathrm{~Hz}, 1 \mathrm{H}), 7.41(\mathrm{~d}, J=4.0 \mathrm{~Hz}, 2 \mathrm{H}), 7.32-7.29$ $(\mathrm{m}, 1 \mathrm{H}), 4.23(\mathrm{~d}, J=12.0 \mathrm{~Hz}, 1 \mathrm{H}), 4.06(\mathrm{~d}, J=12.0 \mathrm{~Hz}, 1 \mathrm{H}), 3.98(\mathrm{~s}, 2 \mathrm{H})$, $3.69(\mathrm{~d}, J=12.0 \mathrm{~Hz}, 1 \mathrm{H}), 3.18(\mathrm{~d}, J=12.0 \mathrm{~Hz}, 1 \mathrm{H}), 2.47$ (s, 3H), 1.05 (s, 3H).

${ }^{13} \mathrm{C}\left\{{ }^{1} \mathrm{H}\right\}$ NMR (101 MHz, $\left.\mathrm{CDCl}_{3}\right): \delta 149.9,148.0,147.1,144.5,138.2,135.1$, $131.5,130.0,128.0,123.4,90.6,79.8,61.4,59.1,47.4,22.5,21.6$. IR (neat): $v=3665,3040,2930$, 2378, 1724, 1553, 1461, 1347, 1284, 1162, 820, 665, 591, $548 \mathrm{~cm}^{-1}$; HRMS (ESI) m/z: [M+Na] ${ }^{+}$ calcd for $\mathrm{C}_{19} \mathrm{H}_{20} \mathrm{IN}_{3} \mathrm{NaO}_{4} \mathrm{~S}^{+}$536.0111; found 536.0117.

\section{(Z)-4-(iodo(phenyl)methylene)-3-methyl-3-(nitromethyl)-1-(phenylsulfonyl) pyrrolidine (4p)} White solid (33 mg, 66\% yield); $\mathrm{R}_{f}=0.25$ (ethyl acetate/petroleum ether, $1: 3$ );<smiles>CC1(C[N+](=O)[O-])CN(S(=O)(=O)c2ccccc2)C/C1=C(/I)c1ccccc1</smiles>
${ }^{1} \mathrm{H}$ NMR (400 MHz, $\left.\mathrm{CDCl}_{3}\right): \delta 7.89(\mathrm{~d}, J=4.0 \mathrm{~Hz}, 2 \mathrm{H}), 7.69(\mathrm{~d}, J=4.0 \mathrm{~Hz}$, $1 \mathrm{H}), 7.64(\mathrm{~d}, J=4.0 \mathrm{~Hz}, 2 \mathrm{H}), 7.39-7.29(\mathrm{~m}, 3 \mathrm{H}), 7.15(\mathrm{~d}, J=4.0 \mathrm{~Hz}, 2 \mathrm{H})$, $4.18(\mathrm{~d}, J=16.0 \mathrm{~Hz}, 1 \mathrm{H}), 4.05(\mathrm{~d}, J=16.0 \mathrm{~Hz}, 1 \mathrm{H}), 3.99$ (d, $J=12.0 \mathrm{~Hz}$, $1 \mathrm{H}), 3.92(\mathrm{~d}, J=16.0 \mathrm{~Hz}, 1 \mathrm{H}), 3.63(\mathrm{~d}, J=8.0 \mathrm{~Hz}, 1 \mathrm{H}), 3.25(\mathrm{~d}, J=12.0 \mathrm{~Hz}$, $1 \mathrm{H}), 1.10(\mathrm{~s}, 3 \mathrm{H}) .{ }^{13} \mathrm{C}\left\{{ }^{1} \mathrm{H}\right\}$ NMR $\left(101 \mathrm{MHz}, \mathrm{CDCl}_{3}\right): \delta 144.6,142.0,134.8$, 133.4, 129.4, 129.1,128.7, 128.0, 127.5, 95.2, 79.7, 61.2, 59.3, 47.0, 22.5. IR (neat): $v=3665,3298$, 2380, 1706, 1443, 1164, 1089, 1047, 826, 721, $600 \mathrm{~cm}^{-1}$; HRMS (ESI) m/z: $[\mathrm{M}+\mathrm{Na}]^{+}$calcd for $\mathrm{C}_{19} \mathrm{H}_{19} \mathrm{IN}_{2} \mathrm{NaO}_{4} \mathrm{~S}^{+}$521.0002; found 520.9996 .

(Z)-4-(iodo(phenyl)methylene)-3-methyl-1-(naphthalen-2-ylsulfonyl)-3(nitromethyl)pyrrolidine (4q) 


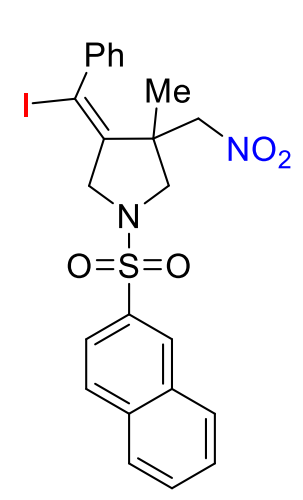

Yellow gum ( $45 \mathrm{mg}, 62 \%$ yield); $\mathrm{R}_{f}=0.25$ (ethyl acetate/petroleum ether, 1:6); ${ }^{1} \mathrm{H}$ NMR (400 MHz, $\left.\mathrm{CDCl}_{3}\right): \delta 8.47(\mathrm{~s}, 1 \mathrm{H}), 8.06(\mathrm{~d}, J=8.0 \mathrm{~Hz}$, $2 \mathrm{H}), 7.97(\mathrm{~d}, J=8.0 \mathrm{~Hz}, 1 \mathrm{H}), 7.87(\mathrm{~d}, J=8.0 \mathrm{~Hz}, 1 \mathrm{H}), 7.72-7.64(\mathrm{~m}, 2 \mathrm{H})$, 7.34-7.29 (m, 3H), 7.12 (d, $J=8.0 \mathrm{~Hz}, 2 \mathrm{H}), 4.20$ (d, $J=16.0 \mathrm{~Hz}, 1 \mathrm{H}), 4.10$ (d, $J=12.0 \mathrm{~Hz}, 1 \mathrm{H}), 4.01(\mathrm{~d}, J=12.0 \mathrm{~Hz}, 1 \mathrm{H}), 3.81-3.72(\mathrm{~m}, 2 \mathrm{H}), 3.30(\mathrm{~d}$, $J=8.0 \mathrm{~Hz}, 1 \mathrm{H}), 1.07(\mathrm{~s}, 3 \mathrm{H}) .{ }^{13} \mathrm{C}\left\{{ }^{1} \mathrm{H}\right\} \mathrm{NMR}\left(101 \mathrm{MHz}, \mathrm{CDCl}_{3}\right): \delta 144.5$, $141.9,135.0,132.1,131.7,129.5,129.4,129.3,129.1,129.0,127.7,127.4$, 123.0, 95.3 79.7, 61.2, 59.3, 47.0, 22.3. IR (neat): $v=3438,2975,1765$, 1451, 1348, 1163, 1041, 822, 747, 665, 589, $550 \mathrm{~cm}^{-1}$; HRMS (ESI) m/z: $[\mathrm{M}+\mathrm{H}]^{+}$calcd for $\mathrm{C}_{23} \mathrm{H}_{22} \mathrm{IN}_{2} \mathrm{O}_{4} \mathrm{~S}^{+}$549.0339; found 549.0338.

(Z)-1-((4-bromophenyl)sulfonyl)-4-(iodo(phenyl)methylene)-3-methyl-3(nitromethyl)pyrrolidine (4r)

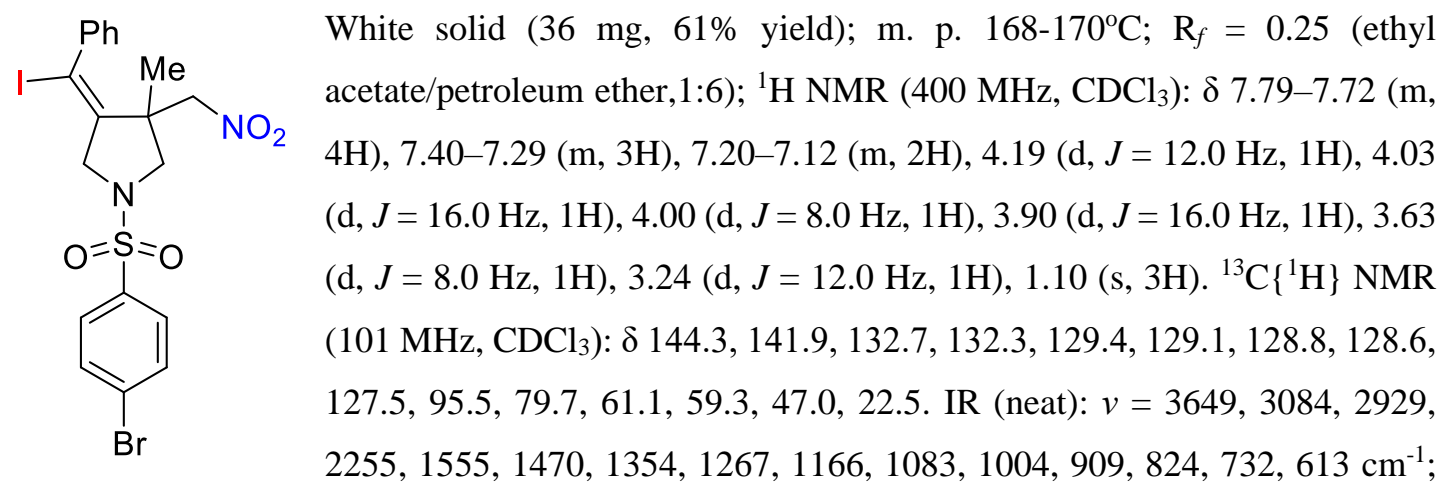

HRMS (ESI) m/z: [M+Na] ${ }^{+}$calcd for $\mathrm{C}_{19} \mathrm{H}_{18} \mathrm{BrIN}_{2} \mathrm{NaO}_{4} \mathrm{~S}^{+}$598.9108; found 598.9110 .

(Z)-4-(iodo(phenyl)methylene)-3-(nitromethyl)-3-phenyl-1-tosylpyrrolidine (4s)

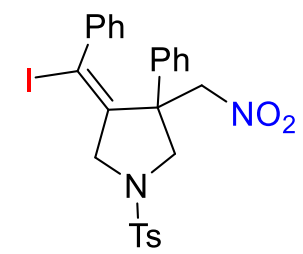

White solid (20.6 mg, 36\% yield); m. p. $127-129^{\circ} \mathrm{C} ; \mathrm{R}_{f}=0.25$ (ethyl acetate/petroleum ether, $1: 6) ;{ }^{1} \mathrm{H}$ NMR (400 MHz, $\left.\mathrm{CDCl}_{3}\right): \delta 7.75$ (d, $J=8.0$ $\mathrm{Hz}, 2 \mathrm{H}), 7.40(\mathrm{~d}, J=8.0 \mathrm{~Hz}, 3 \mathrm{H}), 7.25-7.18(\mathrm{~m}, 3 \mathrm{H}), 7.17-7.09(\mathrm{~m}, 2 \mathrm{H})$, 7.07-6.99 (m, 2H), 6.98-6.92 (m, 2H), 4.65 (d, $J=12.0 \mathrm{~Hz}, 1 \mathrm{H}), 4.35(\mathrm{~d}, J$ $=16.0 \mathrm{~Hz}, 2 \mathrm{H}), 4.01(\mathrm{~d}, J=12.0 \mathrm{~Hz}, 1 \mathrm{H}), 3.94(\mathrm{~d}, J=8.0 \mathrm{~Hz}, 1 \mathrm{H}), 3.87(\mathrm{~d}$, $J=8.0 \mathrm{~Hz}, 1 \mathrm{H}), 2.49$ (s, 3H). ${ }^{13} \mathrm{C}\left\{{ }^{1} \mathrm{H}\right\} \operatorname{NMR}\left(101 \mathrm{MHz}, \mathrm{CDCl}_{3}\right): \delta 145.4,144.3,141.4,141.2,131.7$, $129.9,128.8,128.5,128.1,128.0,127.8,127.4,125.6,95.9,77.2,61.8,61.5,52.5,21.7$. IR (neat): $v=3650,3299,2382,1553,1346,1161,1088,907,819,758,698,665,597 \mathrm{~cm}^{-1} ;$ HRMS (ESI) m/z: $[\mathrm{M}+\mathrm{Na}]^{+}$calcd for $\mathrm{C}_{25} \mathrm{H}_{23} \mathrm{IN}_{2} \mathrm{NaO}_{4} \mathrm{~S}^{+}$597.0315; found 597.0315.

methyl (Z)-4-(iodo(phenyl)methylene)-3-(nitromethyl)-1-tosylpyrrolidine-3-carboxylate (4t) 


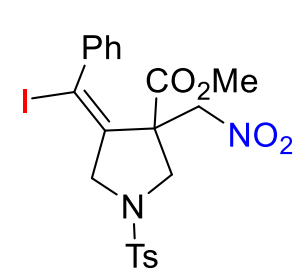

White gum (25 mg, 45\% yield); $\mathrm{R}_{f}=0.25$ (ethyl acetate/petroleum ether, 1:6); ${ }^{1} \mathrm{H}$ NMR (400 MHz, $\left.\mathrm{CDCl}_{3}\right): \delta 7.77(\mathrm{~d}, J=8.0 \mathrm{~Hz}, 2 \mathrm{H}), 7.41(\mathrm{~d}, J=8.0 \mathrm{~Hz}$, 2H), 7.36-7.29 (m, 3H), $7.05(\mathrm{~d}, J=8.0 \mathrm{~Hz}, 2 \mathrm{H}), 4.59$ (d, $J=16.0 \mathrm{~Hz}, 1 \mathrm{H})$, $4.37(\mathrm{~d}, J=16.0 \mathrm{~Hz}, 1 \mathrm{H}), 4.21(\mathrm{~d}, J=4.0 \mathrm{~Hz}, 1 \mathrm{H}), 4.18(\mathrm{~s}, 1 \mathrm{H}), 3.88$ (d, $J=$ $12.0 \mathrm{~Hz}, 1 \mathrm{H}), 3.81(\mathrm{~d}, 12.0 \mathrm{~Hz}, 1 \mathrm{H}), 3.27(\mathrm{~s}, 3 \mathrm{H}), 2.49(\mathrm{~s}, 3 \mathrm{H}) .{ }^{13} \mathrm{C}\left\{{ }^{1} \mathrm{H}\right\} \mathrm{NMR}$ $\left(101 \mathrm{MHz} \mathrm{CDCl}_{3}\right): \delta 168.5,144.5,141.1,139.8,131.8,130.0,129.2,128.7,127.9,127.2,98.1$, 73.6, 60.6, 57.0, 54.8, 53.1, 21.6. IR (neat): $v=3616,3058,2957,1740,1597,1559,1441,1350$, 1242, 1164, 1095, 818, 667, $592 \mathrm{~cm}^{-1}$; HRMS (ESI) m/z: $[\mathrm{M}+\mathrm{Na}]^{+}$calcd for $\mathrm{C}_{21} \mathrm{H}_{21} \mathrm{IN}_{2} \mathrm{NaO}_{6} \mathrm{~S}^{+}$ 579.0057; found 579.0049 .

(Z)-4-(iodo(p-tolyl)methylene)-3-methyl-3-(nitromethyl)-1-tosylpyrrolidine (4u).

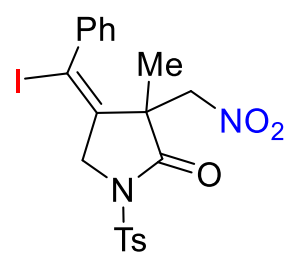

White solid (47.9 mg, 91\% yield); m. p. 205-207 ${ }^{\circ}$; $\mathrm{R}_{f}=0.25$ (ethyl acetate/petroleum ether, $1: 6) ;{ }^{1} \mathrm{H} \mathrm{NMR}\left(400 \mathrm{MHz}, \mathrm{CDCl}_{3}\right): \delta 7.98(\mathrm{~d}, J=8.0$ $\mathrm{Hz}, 2 \mathrm{H}), 7.39-7.32(\mathrm{~m}, 5 \mathrm{H}), 7.10(\mathrm{~s}, 2 \mathrm{H}), 4.61(\mathrm{~d}, J=16.0 \mathrm{~Hz}, 1 \mathrm{H}), 4.42$ (d, $J=12.0 \mathrm{~Hz}, 1 \mathrm{H}), 4.33(\mathrm{~d}, J=16.0 \mathrm{~Hz}, 1 \mathrm{H}), 3.75(\mathrm{~d}, J=16.0 \mathrm{~Hz}, 1 \mathrm{H}), 2.45$ $(\mathrm{s}, 3 \mathrm{H}), 1.19(\mathrm{~s}, 3 \mathrm{H}) .{ }^{13} \mathrm{C}\left\{{ }^{1} \mathrm{H}\right\} \mathrm{NMR}\left(101 \mathrm{MHz}, \mathrm{CDCl}_{3}\right): \delta$ 174.0, 145.8, 141.0, $137.6,133.7,129.7,129.4,128.8,128.3,127.5,97.5,77.7,57.9,50.6,22.0,21.8$. IR (neat): $v=$ 3664, 2927, 2259, 1741, 1555, 1452, 1412, 1368, 1175, 903, 814, $664 \mathrm{~cm}^{-1}$; HRMS (ESI) m/z: $[\mathrm{M}+\mathrm{H}]^{+}$calcd for $\mathrm{C}_{20} \mathrm{H}_{20} \mathrm{IN}_{2} \mathrm{O}_{5} \mathrm{~S}^{+}$527.0132; found 527.0140.

\section{(Z)-4-(1-iodoethylidene)-3-methyl-3-(nitromethyl)-1-tosylpyrrolidine (4v)}

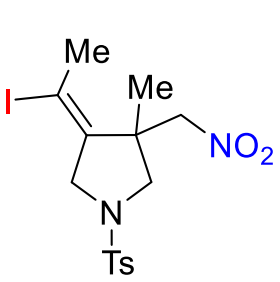

Yellow oil (20 mg, 44\% yield); $\mathrm{R}_{f}=0.25$ (ethyl acetate/petroleum ether, 1:6); ${ }^{1} \mathrm{H}$ NMR (400 MHz, $\left.\mathrm{CDCl}_{3}\right): \delta 7.72(\mathrm{~d}, J=8.0 \mathrm{~Hz}, 2 \mathrm{H}), 7.38(\mathrm{~d}, J=8.0 \mathrm{~Hz}$, $2 \mathrm{H}), 4.55(\mathrm{~d}, J=12.0 \mathrm{~Hz}, 1 \mathrm{H}), 4.48(\mathrm{~d}, J=12.0 \mathrm{~Hz}, 1 \mathrm{H}), 3.91(\mathrm{~d}, J=12.0$ $\mathrm{Hz}, 1 \mathrm{H}), 3.87$ (d, $J=12.0 \mathrm{~Hz}, 1 \mathrm{H}), 3.61(\mathrm{~d}, J=16.0 \mathrm{~Hz}, 1 \mathrm{H}), 2.97$ (d, $J=$ $12.0 \mathrm{~Hz}, 1 \mathrm{H}), 2.59(\mathrm{~s}, 3 \mathrm{H}), 2.44(\mathrm{~s}, 3 \mathrm{H}), 1.41(\mathrm{~s}, 3 \mathrm{H}) .{ }^{13} \mathrm{C}\left\{{ }^{1} \mathrm{H}\right\} \mathrm{NMR}(101$ $\mathrm{MHz}_{\mathrm{CDCl}}$ ): $\delta$ 144.4, 141.7, 131.2, 129.9, 128.1, 95.5, 79.1, 61.5, 59.6, 46.3, 30.4, 21.6, 20.7. IR (neat): $v=3626,2924,1591,1347,1162,1091,1009,870,825,736,664 \mathrm{~cm}^{-1} ; \mathrm{HRMS}(\mathrm{ESI}) \mathrm{m} / \mathrm{z}$ : $[\mathrm{M}+\mathrm{Na}]^{+}$calcd for $\mathrm{C}_{15} \mathrm{H}_{19} \mathrm{IN}_{2} \mathrm{NaO}_{4} \mathrm{~S}^{+}$473.0002; found 472.9996 .

\section{(Z)-4-(iodomethylene)-3-methyl-3-(nitromethyl)-1-tosylpyrrolidine (4w)}

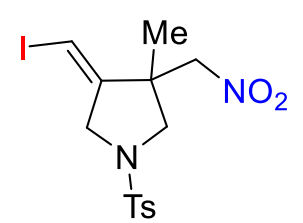

Colorless oil (40 mg, $92 \%$ yield); $\mathrm{R}_{f}=0.25$ (ethyl acetate/ petroleum ether, 1:6); ${ }^{1} \mathrm{H}$ NMR (400 MHz, $\left.\mathrm{CDCl}_{3}\right): \delta 7.73(\mathrm{~d}, J=8.0 \mathrm{~Hz}, 2 \mathrm{H}), 7.38$ (d, $J=$ $8.0 \mathrm{~Hz}, 2 \mathrm{H}), 6.23(\mathrm{t}, J=2.8 \mathrm{~Hz}, 1 \mathrm{H}), 4.40(\mathrm{~d}, J=12.0 \mathrm{~Hz}, 1 \mathrm{H}), 4.36(\mathrm{~d}, J=$ $12.0 \mathrm{~Hz}, 1 \mathrm{H}), 3.91(\mathrm{~d}, J=20.0 \mathrm{~Hz}, 1 \mathrm{H}), 3.78(\mathrm{~d}, J=8.0 \mathrm{~Hz}, 1 \mathrm{H}), 3.69(\mathrm{~d}, J$ $=16.0 \mathrm{~Hz}, 1 \mathrm{H}), 3.05(\mathrm{~d}, J=8.0 \mathrm{~Hz}, 1 \mathrm{H}), 2.45(\mathrm{~s}, 3 \mathrm{H}), 1.30(\mathrm{~s}, 3 \mathrm{H}) .{ }^{13} \mathrm{C}\left\{{ }^{1} \mathrm{H}\right\} \mathrm{NMR}(101 \mathrm{MHz}$, $\left.\mathrm{CDCl}_{3}\right): \delta 150.3,144.4,131.7,130.0,127.9,80.8,74.6,58.0,56.5,47.9,21.6,21.0$. IR (neat): $v=$ 3688, 3298, 2927, 2380, 1551, 1441, 1343, 1260, 1159, 1090, 661, $585 \mathrm{~cm}^{-1}$; HRMS (ESI) m/z: $[\mathrm{M}+\mathrm{H}]^{+}$calcd for $\mathrm{C}_{14} \mathrm{H}_{18} \mathrm{IN}_{2} \mathrm{O}_{4} \mathrm{~S}^{+} 437.0026$; found 437.0036 . 
methyl (Z)-4-(iodomethylene)-3-(nitromethyl)-1-tosylpyrrolidine-3-carboxylate (4x)<smiles>COC1(C(=O)[N+](=O)[O-])CN([13CH3])CC1=CI</smiles>

Colorless solid ( $36 \mathrm{mg}, 75 \%$ yield); m. p. $136-138^{\circ} \mathrm{C} ; \mathrm{R}_{f}=0.25$ (ethyl acetate/ petroleum ether, 1:6); ${ }^{1} \mathrm{H}$ NMR (400 MHz, $\left.\mathrm{CDCl}_{3}\right): \delta 7.74(\mathrm{~d}, J=8.0 \mathrm{~Hz}, 2 \mathrm{H})$, $7.40(\mathrm{~d}, J=8.0 \mathrm{~Hz}, 2 \mathrm{H}), 6.61(\mathrm{t}, J=2.8 \mathrm{~Hz}, 1 \mathrm{H}), 4.83(\mathrm{~d}, J=16.0 \mathrm{~Hz}, 1 \mathrm{H})$, $4.55(\mathrm{~d}, J=16.0 \mathrm{~Hz}, 1 \mathrm{H}), 4.07(\mathrm{~d}, J=12.0 \mathrm{~Hz}, 1 \mathrm{H}), 3.87(\mathrm{~d}, J=1.4 \mathrm{~Hz}, 2 \mathrm{H})$, $3.74(\mathrm{~s}, 3 \mathrm{H}), 3.51(\mathrm{~d}, J=16.0 \mathrm{~Hz}, 1 \mathrm{H}), 2.47(\mathrm{~s}, 3 \mathrm{H}) .{ }^{13} \mathrm{C}\left\{{ }^{1} \mathrm{H}\right\} \mathrm{NMR}(101 \mathrm{MHz}$, $\left.\mathrm{CDCl}_{3}\right): \delta 168.2,144.7,143.7,131.4,130.1,128.0,77.9,76.4,56.4,55.9,54.7,53.7,21.6$. IR (neat): $v=3683,2960,1742,1559,1349,1295,1164,817,664,591,547 \mathrm{~cm}^{-1} ;$ HRMS (ESI) m/z: $[\mathrm{M}+\mathrm{Na}]^{+}$ calcd for $\mathrm{C}_{15} \mathrm{H}_{17} \mathrm{IN}_{2} \mathrm{NaO}_{6} \mathrm{~S}^{+}$502.9744; found 502.9742 .

(Z)-4-(iodomethylene)-3-methyl-3-(nitromethyl)-1-tosylpyrrolidin-2-one (4y)

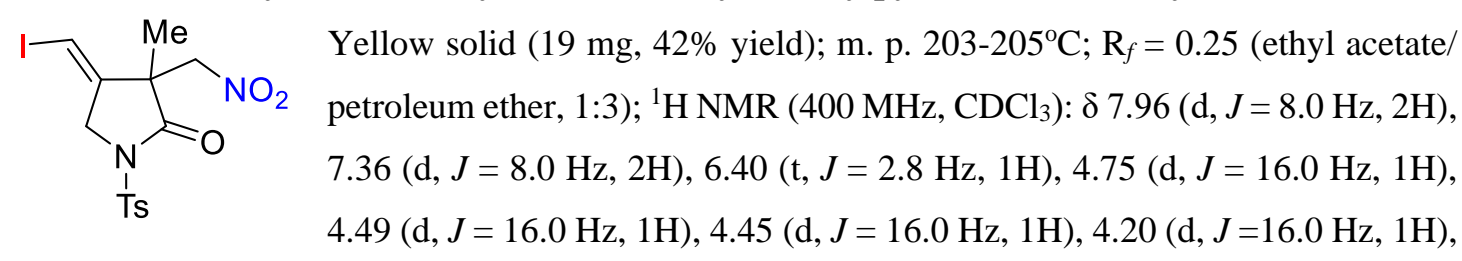
2.44 (s, 3H), 1.32 (s, 3H). ${ }^{13} \mathrm{C}\left\{{ }^{1} \mathrm{H}\right\}$ NMR (101 MHz, $\left.\mathrm{CDCl}_{3}\right): \delta 173.1,145.9,143.9,133.8,129.8$, 128.3, 78.9, 75.8, 55.0, 51.0, 23.2, 21.8. IR (neat): $v=3660,2935,2260,1755,1555,1456,1422$, 1368, 1173, 906, 812, $668 \mathrm{~cm}^{-1}$; HRMS (ESI) m/z: $[\mathrm{M}+\mathrm{Na}]^{+}$calcd for $\mathrm{C}_{14} \mathrm{H}_{15} \mathrm{IN}_{2} \mathrm{NaO}_{5} \mathrm{~S}^{+} 472.9639$; found 472.9641 .

\section{Procedure for the scale-up reaction}

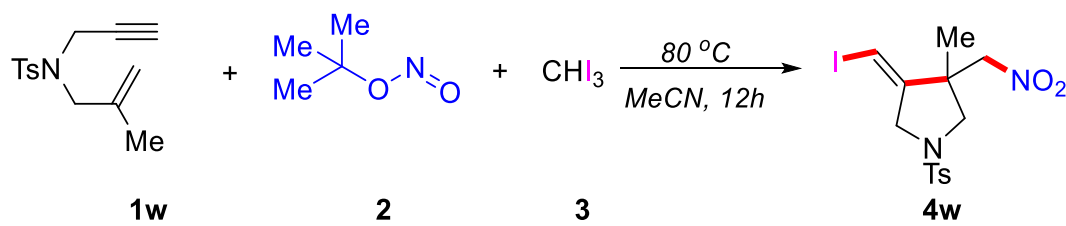

Enynes 1 w (1.32g, $5.0 \mathrm{mmol}, 1.0$ equiv.), $\mathrm{CHI}_{3}$ ( $3.9 \mathrm{~g}$, 2.0 equiv.), and a stir bar were added to a sealed $250 \mathrm{~mL}$ round-bottomed flask under a nitrogen atmosphere, $\mathrm{MeCN}(100 \mathrm{~mL})$ as solvent was then added. Then $2(0.93 \mathrm{~g}, 9.0 \mathrm{mmol}, 1.8$ equiv. $)$ was added, the mixture was stirred for $12 \mathrm{~h}$ at $80^{\circ} \mathrm{C}$ with an oil bath. The crude mixture was concentrated in vacuo and then purified by flash column chromatography on silica gel (EtOAc/petroleum ether 1:6) to give the desired product $\mathbf{4} \mathbf{w}$ as colorless solid (1.4 g, 65\% yield).

\section{General procedure for the product derivation $5 \mathrm{a}-5 \mathrm{c}$}

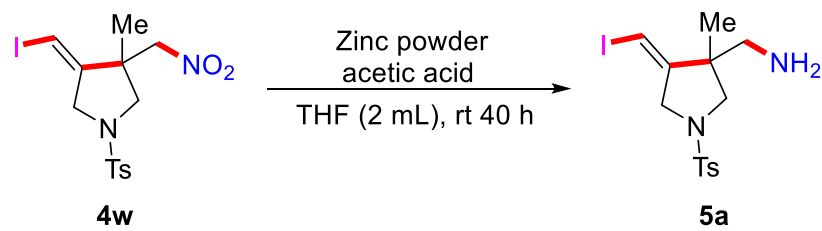


Compound 4w $87.3 \mathrm{mg}$ (0.2 mmol, 1.0 equiv.), Zinc powder $31.2 \mathrm{mg}(0.48 \mathrm{mmol}, 2.4$ equiv.), acetic acid $(0.6 \mathrm{~mL})$, and a stir bar were added to a sealed tube under a nitrogen atmosphere, THF $(2.0 \mathrm{~mL})$ as the solvent were added. The mixture was stirred overnight at room temperature with an oil bath. The crude mixture was directly purified by flash column chromatography on silica gel (EtOAc/apetroleum ether $=2: 1)$ to give the desired product $\mathbf{5 a}$ as yellow gum $(65 \mathrm{mg}, 80 \%$ yield); $\mathrm{R}_{f}=0.25$ (ethyl acetate/ petroleum ether, $\left.1: 1\right) ;{ }^{1} \mathrm{H}$ NMR (400 MHz, DMSO- $\left.\mathrm{d}_{6}\right): \delta 7.69$ (d, $J=8.0$ $\mathrm{Hz}, 2 \mathrm{H}), 7.47$ (d, $J=8.0 \mathrm{~Hz}, 2 \mathrm{H}), 6.41(\mathrm{t}, J=2.4 \mathrm{~Hz}, 1 \mathrm{H}), 3.68$ (d, $J=16.0 \mathrm{~Hz}, 1 \mathrm{H}), 3.58$ (d, $J=$ $12.0 \mathrm{~Hz}, 1 \mathrm{H}), 3.49(\mathrm{~d}, J=12.0 \mathrm{~Hz}, 1 \mathrm{H}), 2.95(\mathrm{~d}, J=8.0 \mathrm{~Hz}, 1 \mathrm{H}), 2.63(\mathrm{q}, J=12.0 \mathrm{~Hz}, 2 \mathrm{H}), 2.41(\mathrm{~s}$, 3H), 1.86 (s, 2H),1.06 (s, 3H). ${ }^{13} \mathrm{C}\left\{{ }^{1} \mathrm{H}\right\}$ NMR (101 MHz, DMSO): $\delta 153.0,143.9,131.7,130.0$, 127.6, 73.9, 57.7, 56.9, 49.5, 47.5, 21.2, 21.0. IR (neat): $v=3434,2253,2126,1656,1343,1028$, $1003,825,764,626 \mathrm{~cm}^{-1}$; HRMS (ESI) m/z: $[\mathrm{M}+\mathrm{Na}]^{+}$calcd for $\mathrm{C}_{14} \mathrm{H}_{19} \mathrm{IN}_{2} \mathrm{NaO}_{2} \mathrm{~S}^{+} 429.0104$; found 429.0108 .
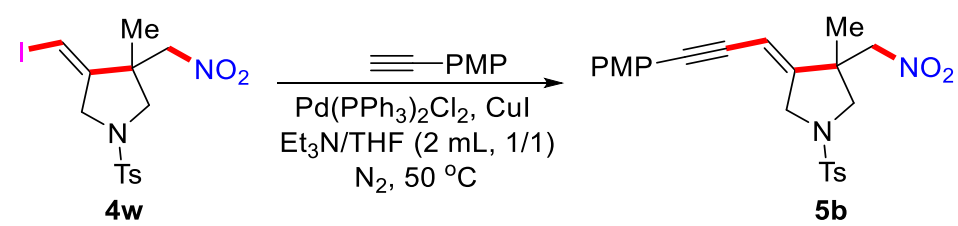

Compound 4w $87.3 \mathrm{mg}$ (0.2 mmol, 1.0 equiv.), $\mathrm{Pd}\left(\mathrm{PPh}_{3}\right)_{2} \mathrm{Cl}_{2}(0.004 \mathrm{mmol}), \mathrm{CuI}(0.004 \mathrm{mmol})$, and a stir bar were added to a sealed tube under a nitrogen atmosphere, $\mathrm{Et}_{3} \mathrm{~N}(1.0 \mathrm{~mL})$, THF $(1.0 \mathrm{~mL})$ as solvent were added. Then 1-ethynyl-4-methoxybenzene $31.7 \mathrm{mg}(0.24 \mathrm{mmol}, 1.2$ equiv.) was added, the mixture was stirred for $12 \mathrm{~h}$ at $50{ }^{\circ} \mathrm{C}$ with an oil bath. The crude mixture was directly purified by flash column chromatography on silica gel (EtOAc/petroleum ether $=1: 3)$ to give the desired product $\mathbf{5 b}$ as yellow gum ( $60 \mathrm{mg}, 67 \%$ yield); $\mathrm{R}_{f}=0.25$ (ethyl acetate/ petroleum ether, 1:6); ${ }^{1} \mathrm{H}$ NMR (400 MHz, $\left.\mathrm{CDCl}_{3}\right): \delta 7.74(\mathrm{~d}, J=8.0 \mathrm{~Hz}, 2 \mathrm{H}), 7.37-7.34(\mathrm{~m}, 4 \mathrm{H}), 6.86(\mathrm{~d}, J=12.0 \mathrm{~Hz}$, 2H), 5.58 (t, $J=2.8 \mathrm{~Hz}, 1 \mathrm{H}), 4.40$ (d, $J=12.0 \mathrm{~Hz}, 1 \mathrm{H}), 4.33$ (d, $J=12.0 \mathrm{~Hz}, 1 \mathrm{H}), 4.18$ (d, $J=16.0$ $\mathrm{Hz}, 1 \mathrm{H}), 4.03$ (d, $J=20.0 \mathrm{~Hz}, 1 \mathrm{H}), 3.82(\mathrm{~s}, 3 \mathrm{H}), 3.70$ (d, $J=8.0 \mathrm{~Hz}, 1 \mathrm{H}), 3.00$ (d, $J=8.0 \mathrm{~Hz}, 1 \mathrm{H})$, $2.43(\mathrm{~s}, 3 \mathrm{H}), 1.31(\mathrm{~s}, 3 \mathrm{H}) .{ }^{13} \mathrm{C}\left\{{ }^{1} \mathrm{H}\right\}$ NMR $\left(101 \mathrm{MHz}, \mathrm{CDCl}_{3}\right): \delta 160.0,150.6,144.2,133.0,129.9$, 127.9, 114.4, 114.2, 114.1, 105.3, 97.6, 83.0, 81.1, 57.4, 55.3, 51.5, 46.0, 21.6, 21.2. IR (neat): $v=$ $3639,3151,1715,1551,1381,1248,1161,1043,829,490,451 \mathrm{~cm}^{-1}$; HRMS (ESI) m/z: $[\mathrm{M}+\mathrm{Na}]^{+}$ calcd for $\mathrm{C}_{23} \mathrm{H}_{24} \mathrm{~N}_{2} \mathrm{NaO}_{5} \mathrm{~S}^{+} 463.1298$; found 463.1296 .

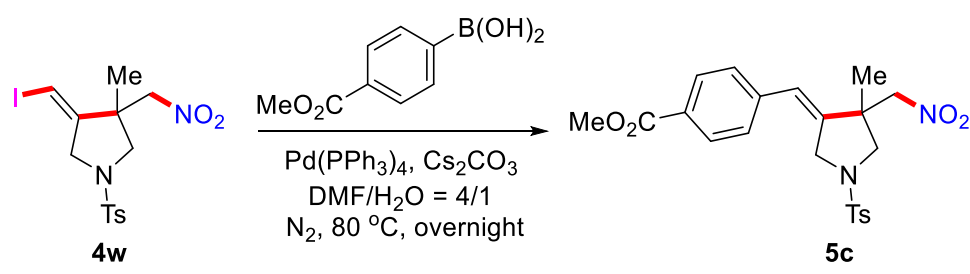


Compound 4w $43.6 \mathrm{mg}$ ( $0.1 \mathrm{mmol}, 1.0$ equiv.), (4-(methoxycarbonyl)phenyl)boronic acid (21.5 mg, $0.12 \mathrm{mmol}, 1.2$ equiv.), $\mathrm{Pd}\left(\mathrm{PPh}_{3}\right)_{4}(13.0 \mathrm{mg}, 0.005 \mathrm{mmol}), \mathrm{Cs}_{2} \mathrm{CO}_{3}(52 \mathrm{mg}, 0.15 \mathrm{mmol})$, and a stir bar were added to a sealed tube under a nitrogen atmosphere, DMF $(1.6 \mathrm{~mL}), \mathrm{H}_{2} \mathrm{O}(0.4 \mathrm{~mL})$ as solvent were added. The mixture was stirred overnight at $80^{\circ} \mathrm{C}$ with an oil bath. The crude mixture was directly purified by flash column chromatography on silica gel (EtOAc/petroleum ether $=1: 2)$ to give the desired product $\mathbf{5 c}$ as colorless oil ( $40 \mathrm{mg}, 90 \%$ yield); $\mathrm{R}_{f}=0.25$ (ethyl acetate/ petroleum ether, 1:3); ${ }^{1} \mathrm{H}$ NMR (400 MHz, $\mathrm{CDCl}_{3}$ ): $\delta 8.02(\mathrm{~d}, J=8.0 \mathrm{~Hz}, 2 \mathrm{H}), 7.74(\mathrm{~d}, J=8.0 \mathrm{~Hz}, 2 \mathrm{H}), 7.35$ $(\mathrm{d}, J=8.0 \mathrm{~Hz}, 2 \mathrm{H}), 7.20(\mathrm{~d}, J=8.0 \mathrm{~Hz}, 2 \mathrm{H}), 6.30(\mathrm{~s}, 1 \mathrm{H}), 4.48(\mathrm{~d}, J=12.0 \mathrm{~Hz}, 1 \mathrm{H}), 4.43(\mathrm{~d}, J=$ $12.0 \mathrm{~Hz}, 1 \mathrm{H}), 4.30(\mathrm{~d}, J=20.0 \mathrm{~Hz}, 1 \mathrm{H}), 4.03(\mathrm{~d}, J=16.0 \mathrm{~Hz}, 1 \mathrm{H}), 3.93(\mathrm{~s}, 3 \mathrm{H}), 3.71(\mathrm{~d}, J=8.0 \mathrm{~Hz}$, $1 \mathrm{H}), 2.97(\mathrm{~d}, J=16.0 \mathrm{~Hz}, 1 \mathrm{H}), 2.43(\mathrm{~s}, 3 \mathrm{H}), 1.40(\mathrm{~s}, 3 \mathrm{H}) .{ }^{13} \mathrm{C}\left\{{ }^{1} \mathrm{H}\right\} \mathrm{NMR}\left(101 \mathrm{MHz}, \mathrm{CDCl}_{3}\right): \delta$ 166.5, 144.3, 141.7, 139.6, 130.0, 129.9, 129.3, 128.2, 127.8, 127.6, 124.1, 81.6, 56.1, 52.2, 50.6, 47.1, 21.6, 21.4. IR (neat): $v=3654,2951,2369,1718,1550,1346,1280,1160,1042,704,663 \mathrm{~cm}^{-}$ ${ }^{1}$; HRMS (ESI) m/z: [M+Na] ${ }^{+}$calcd for $\mathrm{C}_{22} \mathrm{H}_{24} \mathrm{~N}_{2} \mathrm{NaO}_{6} \mathrm{~S}^{+} 467.1247$; found 467.1253.

7. Mechanism proposal

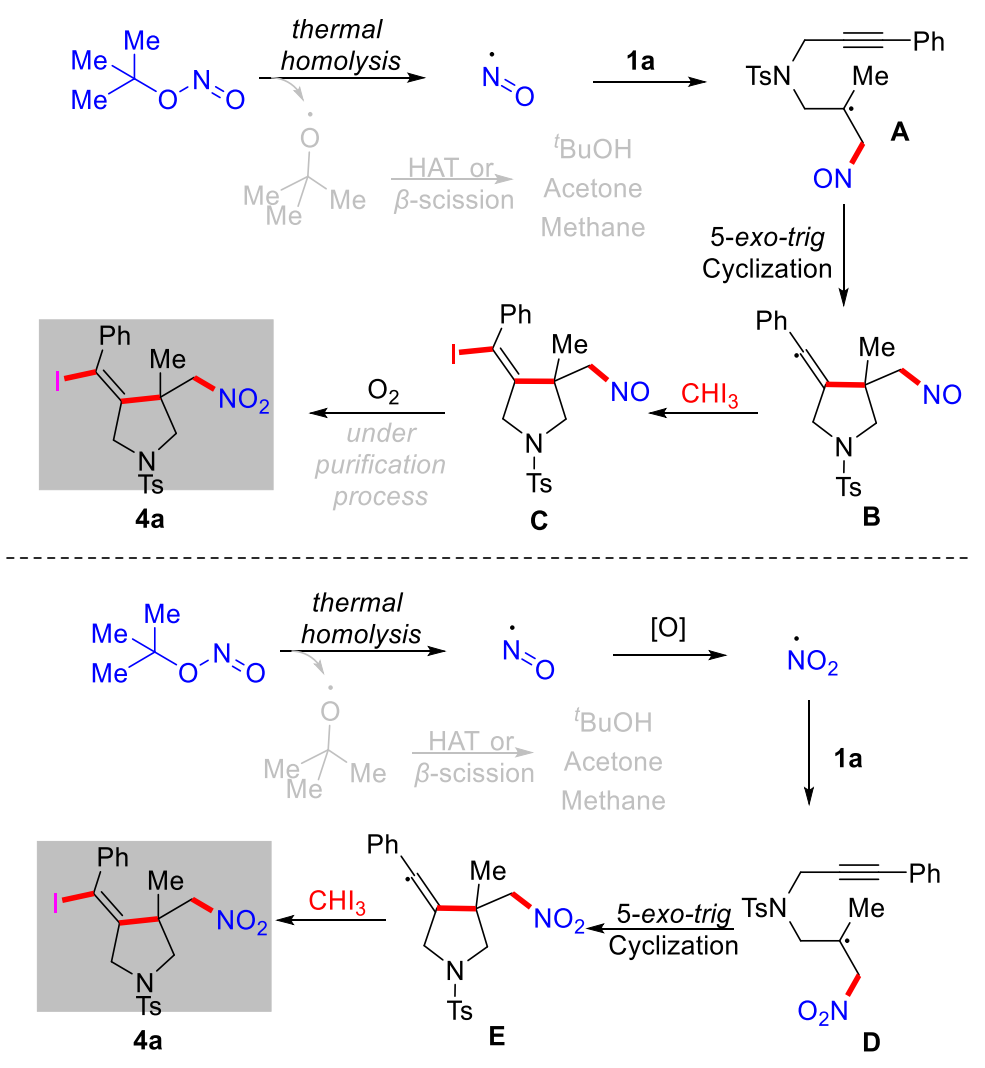




\section{X-ray Structure of products}

Single crystals of $\mathbf{4 a}$ were obtained by recrystallization from dichloromethane/n-hexane solution. The crystal structure was determined by standard crystallographic methods. A colorless blockshaped crystal $\left(0.15 \times 0.12 \times 0.11 \mathrm{~mm}^{3}\right)$ was used for single-crystal X-ray diffraction. The data were collected at $273.15 \mathrm{~K}$ using a Bruker $\mathrm{D}_{8}$ QUEST X-ray diffractometer with graphitemonochromated Mo-K $\alpha$ radiation $(\lambda=0.71073 \AA)$. The structure was solved by Direct Methods and refined by full-matrix least-squares techniques on F2 with anisotropic displacement parameters for all atoms using SHELX-2014 ${ }^{[9]}$. All the processes were performed within Olex2. The final refinements included anisotropic displacements parameters for all atoms and a secondary extinction correction. The crystallographic parameter data is listed in Table S1

Figure S1. Crystal Structure of 4a with thermal ellipsoids drawn at the 30\% probability level. Hydrogens are omitted for clearity and Crystallographic data for $\mathbf{4 a}$ have been deposited with the Cambridge Crystallographic Data Center as CCDC: 2052501. And the crystal data and details of the data collection are given in Table S1.

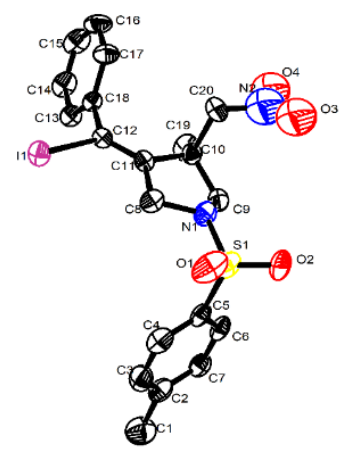

Table S1. Crystal data of $\mathbf{4 a}$

\begin{tabular}{|c|c|c|}
\hline Empirical formula & \multicolumn{2}{|l|}{$\mathrm{C}_{20} \mathrm{H}_{21} \mathrm{IN}_{2} \mathrm{O}_{4} \mathrm{~S}$} \\
\hline CCDC & \multicolumn{2}{|l|}{2052501} \\
\hline Formula weight & \multicolumn{2}{|l|}{512.35} \\
\hline Temperature(K) & \multicolumn{2}{|l|}{273.15} \\
\hline Wavelength $(\AA)$ & \multicolumn{2}{|l|}{0.71073} \\
\hline Crystal system & \multicolumn{2}{|l|}{ triclinic } \\
\hline space group & \multicolumn{2}{|l|}{$\mathrm{P}-1$} \\
\hline Unit cell dimensions & $\begin{array}{l}\mathrm{a}=12.0273(16) \AA \\
\mathrm{b}=12.0988(15) \AA \\
\mathrm{c}=15.469(2) \AA\end{array}$ & $\begin{array}{l}\alpha=86.715(4)^{\circ} \\
\beta=77.907(4)^{\circ} \\
\gamma=76.552(4)^{\circ}\end{array}$ \\
\hline Volume $\left(\AA^{3}\right)$ & \multicolumn{2}{|l|}{$2140.6(5)$} \\
\hline Z & \multicolumn{2}{|l|}{4} \\
\hline
\end{tabular}




\begin{tabular}{|l|l|}
\hline Calculated density $\left(\mathrm{g} \cdot \mathrm{cm}^{-3}\right)$ & 1.590 \\
\hline Absorption coefficient $\left(\mathrm{mm}^{-1}\right)$ & 1.621 \\
\hline $\mathrm{F}(000)$ & 1024.0 \\
\hline Crystal size $(\mathrm{mm})$ & $0.15 \times 0.12 \times 0.11$ \\
\hline$\theta$ range for data collection & 4.002 to 55.23 \\
\hline$h, k, l$ ranges & $-15<=\mathrm{h}<=15,-15<=\mathrm{k}<=15,-20<=1<=19$ \\
\hline Reflections collected & 24864 \\
\hline Independent reflections & $9670[\mathrm{R}(\mathrm{int})=0.0437]$ \\
\hline Completeness & $97 \%$ \\
\hline Absorption correction & $\mathrm{None}$ \\
\hline Data / restraints / parameters & $9670 / 51 / 509$ \\
\hline Goodness-of-fit on $F^{2}$ & 1.017 \\
\hline Final $R$ indices $[\mathrm{I}>2 \sigma(\mathrm{I})]$ & $\mathrm{R}_{1}=0.0659, \mathrm{wR}_{2}=0.1775$ \\
\hline$R$ indices (all data) & $\mathrm{R}_{1}=0.1136, \mathrm{wR}_{2}=0.2018$ \\
\hline Largest diff. peak and hole & 1.25 and $-0.88 \mathrm{e} \cdot \AA^{-3}$ \\
\hline
\end{tabular}

Figure S2. Crystal Structure of $\mathbf{4 w}$ with thermal ellipsoids drawn at the $30 \%$ probability level. Crystallographic data for $\mathbf{4 w}$ have been deposited with the Cambridge Crystallographic Data Center as CCDC: 2074660. And the crystal data and details of the data collection are given in Table S2.

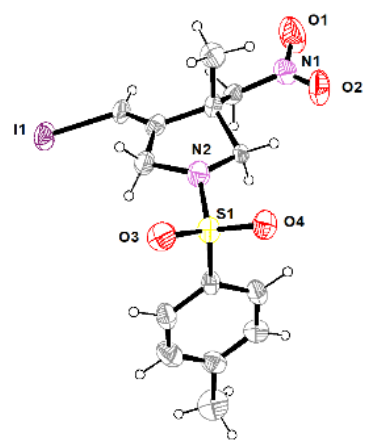

Table S2. Crystal data of $4 \mathrm{w}$

\begin{tabular}{|l|l|}
\hline Empirical formula & $\mathrm{C}_{14} \mathrm{H}_{17} \mathrm{IN}_{2} \mathrm{O}_{4} \mathrm{~S}$ \\
\hline CCDC & 2074660 \\
\hline Formula weight & 436.25 \\
\hline Temperature(K) & 273.15 \\
\hline Wavelength( $(\AA)$ & 0.71073 \\
\hline Crystal system & orthorhombic \\
\hline space group & Pca2 $2_{1}$ \\
\hline
\end{tabular}




\begin{tabular}{|l|l|l|}
\hline Unit cell dimensions & $\begin{array}{l}\mathrm{a}=11.5453(13) \AA \\
\mathrm{b}=9.4644(10) \AA \\
\mathrm{c}=31.833(4) \AA\end{array}$ & $\begin{array}{l}\alpha=90^{\circ} \\
\beta=90^{\circ} \\
\gamma=90^{\circ}\end{array}$ \\
\hline Volume $\left(\AA^{3}\right)$ & $3478.4(7)$ \\
\hline $\mathrm{Z}$ & 8 \\
\hline Calculated density $\left(\mathrm{g} \cdot \mathrm{cm}^{-3}\right)$ & 1.666 \\
\hline Absorption coefficient $\left(\mathrm{mm}^{-1}\right)$ & 1.979 \\
\hline F(000) & 1728.0 \\
\hline Crystal size(mm) & $0.12 \times 0.11 \times 0.1$ \\
\hline$\theta$ range for data collection & 4.304 to 55.048 \\
\hline$h, k, l$ ranges & $-14 \leq \mathrm{h} \leq 13,-12 \leq \mathrm{k} \leq 12,-41 \leq 1 \leq 34$ \\
\hline Reflections collected & 35137 \\
\hline Independent reflections & $6793\left[\mathrm{R}_{\text {int }}=0.0895\right]$ \\
\hline Completeness & $1.66 / 0.85$ \\
\hline Absorption correction & Multi-scan \\
\hline Data / restraints / parameters & $6793 / 337 / 476$ \\
\hline Goodness-of-fit on $F^{2}$ & 1.024 \\
\hline Final $R$ indices $[\mathrm{I}>2 \sigma(\mathrm{I})]$ & $\mathrm{R}_{1}=0.0641, \mathrm{wR}_{2}=0.1352$ \\
\hline$R$ indices (all data) & $\mathrm{R}_{1}=0.1370, \mathrm{wR}_{2}=0.1589$ \\
\hline Largest diff. peak and hole & 0.76 and $-1.13 \mathrm{e} \cdot \AA^{-3}$ \\
\hline
\end{tabular}




\section{References:}

[1] Ye, K.-Y. Song, Z. Sauer, G. S. Harenberg, J. H. Fu, N. Lin, S. Synthesis of Chlorotrifluoromethylated Pyrrolidines by Electrocatalytic Radical Ene-Yne Cyclization. Chem. Eur. J. 2018, 24, 12274-12279.

[2] Gao, P. Yan, X.-B. Tao, T. Yang, F. He, T. Song, X.-R. Liu, X.-Y. Liang, Y.-M. Coppercatalyzed trifluoromethylation-cyclization of enynes: highly regioselective construction of trifluoromethylated carbocycles and heterocycles. Chem. Eur. J. 2013, 19, 14420-14424.

[3] Liu, R. Yang, D. Chang, F. Giordano, L. Liu, G. Tenaglia, A. An Electrophilic Ruthenium Complex that Enables the Cycloisomerization of 1,6-Enynes to Access Azabicyclo[4.1.0]heptenes. Asian J. Org. Chem. 2019, 8, 2011-2016.

[4] Wang, Y.-Q. He, Y.-T. Zhang, L.-L. Wu, X.-X. Liu, X.-Y. Liang, Y.-M. Palladium-Catalyzed Radical Cascade Iododifluoromethylation/Cyclization of 1,6-Enynes with Ethyl Difluoroiodoacetate. Org. Lett., 2015, 17, 4280-4283

[5] Zhu, S. Yang, H. Jiang, A. Zhou, B. Han, Y. Yan, C. Shi, Y. Hou, H. Copper-Catalyzed Bromodifluoroacetylative Cyclization of Enynes. J. Org. Chem. 2020, 85, 15667-15675.

[6] Hou, H. Tang, D. Li, H. Xu, Y. Yan, C. Shi, Y. Chen, X. Zhu, S. Visible-light driven Chlorotrifluoromethylative and Chlorotrichloromethylative Cyclizations of Enynes. J. Org. Chem. 2019, 84, 7509-7517.

[7] Liu, B. Song, R.-J. Ouyang, X.-H. Li, Y. Hu, M. Li, J.-H. Palladium-catalyzed oxidative 6-exotrig cyclization of 1,6-enynes: facile synthesis of bicyclo[4.1.0]heptan-5-ones. Chem. Commun., 2015, 51, 12819-12822

[8] Gao, M. Gao, Q. Hao, X. Wu, Y. Zhang, Q. Liu, G. Liu, R. Ruthenium Carbene-Mediated Construction of Strained Allenes via the Enyne Cross-Metathesis/Cyclopropanation of 1,6-Enynes. Org. Lett. 2020, 22, 1139-1143.

[9] Dolomanov, O. V. Bourhis, L. J. Gildea, R. J. Howard, J. A. K. Puschmann, H. OLEX2: A Complete Structure Solution, Refinement and Analysis Program. J. Appl. Crystallogr. 2009, 42, 339-341. 


$$
\text { i }
$$

10. ${ }^{1} \mathrm{H},{ }^{13} \mathrm{C}\{1 \mathrm{H}\}$ and ${ }^{19} \mathrm{~F}\left\{{ }^{1} \mathrm{H}\right\}$ NMR spectra
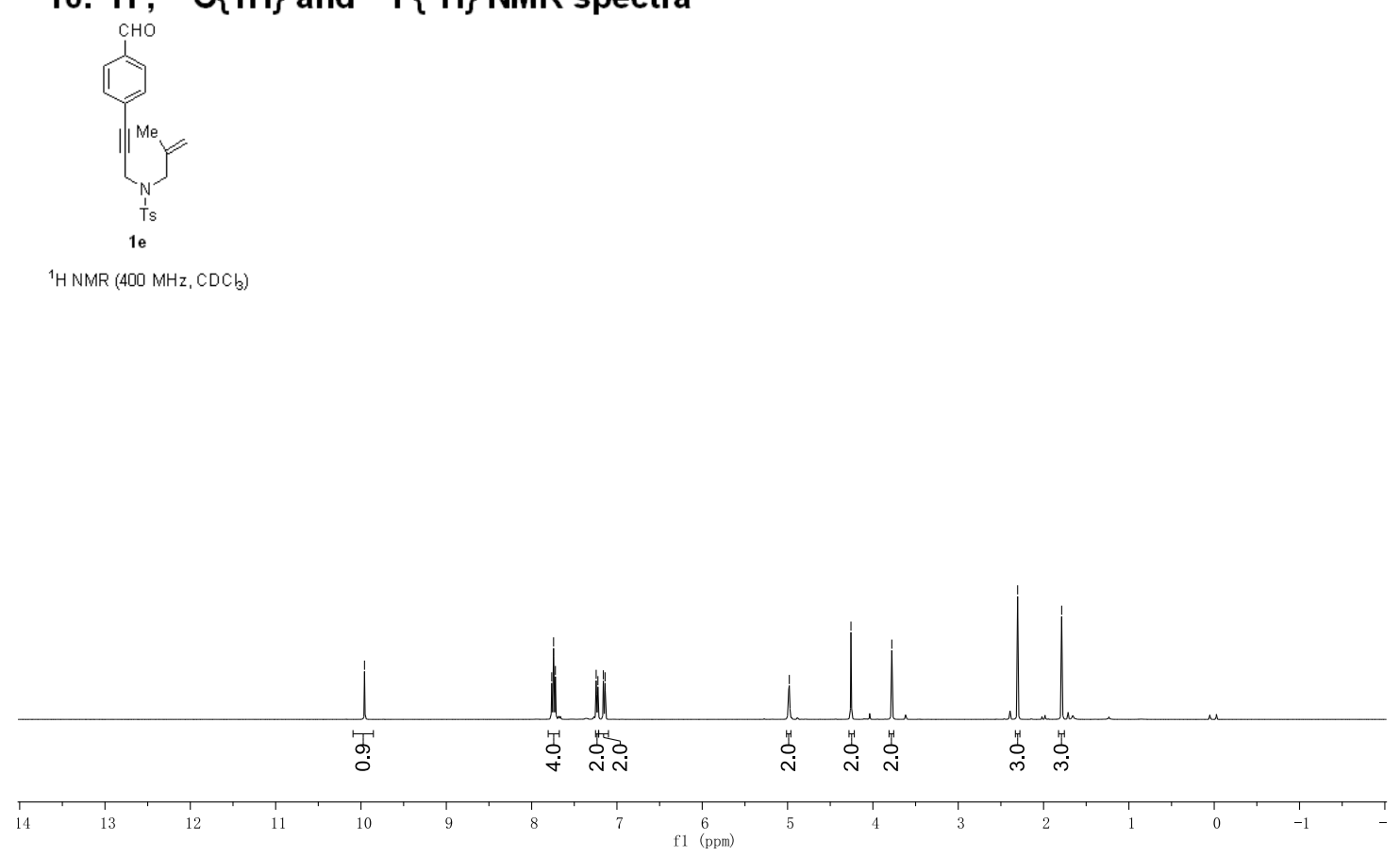

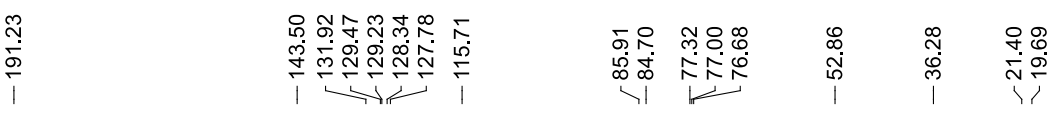
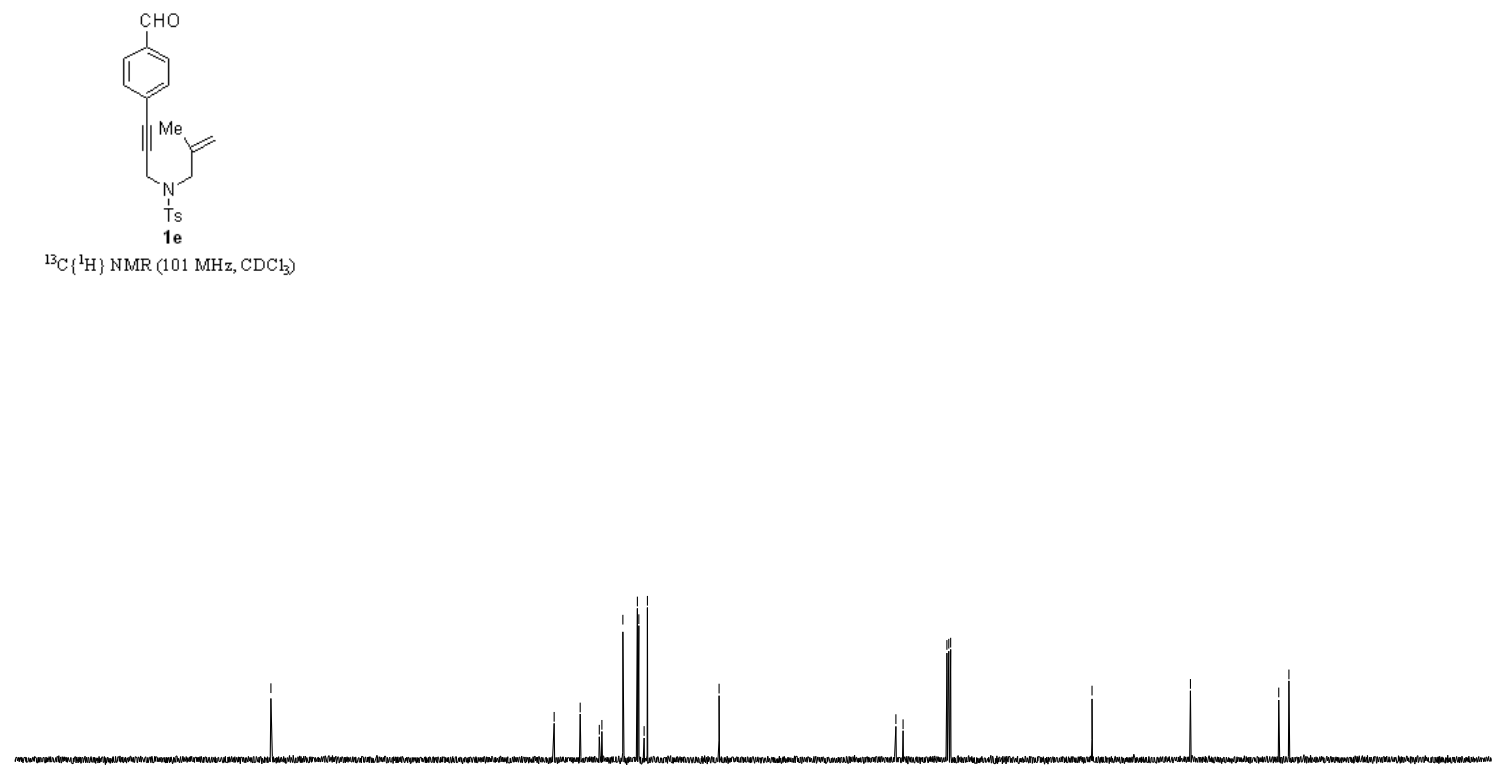

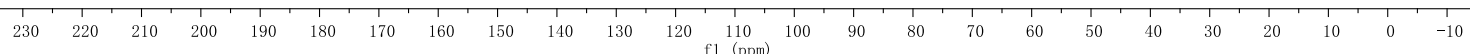



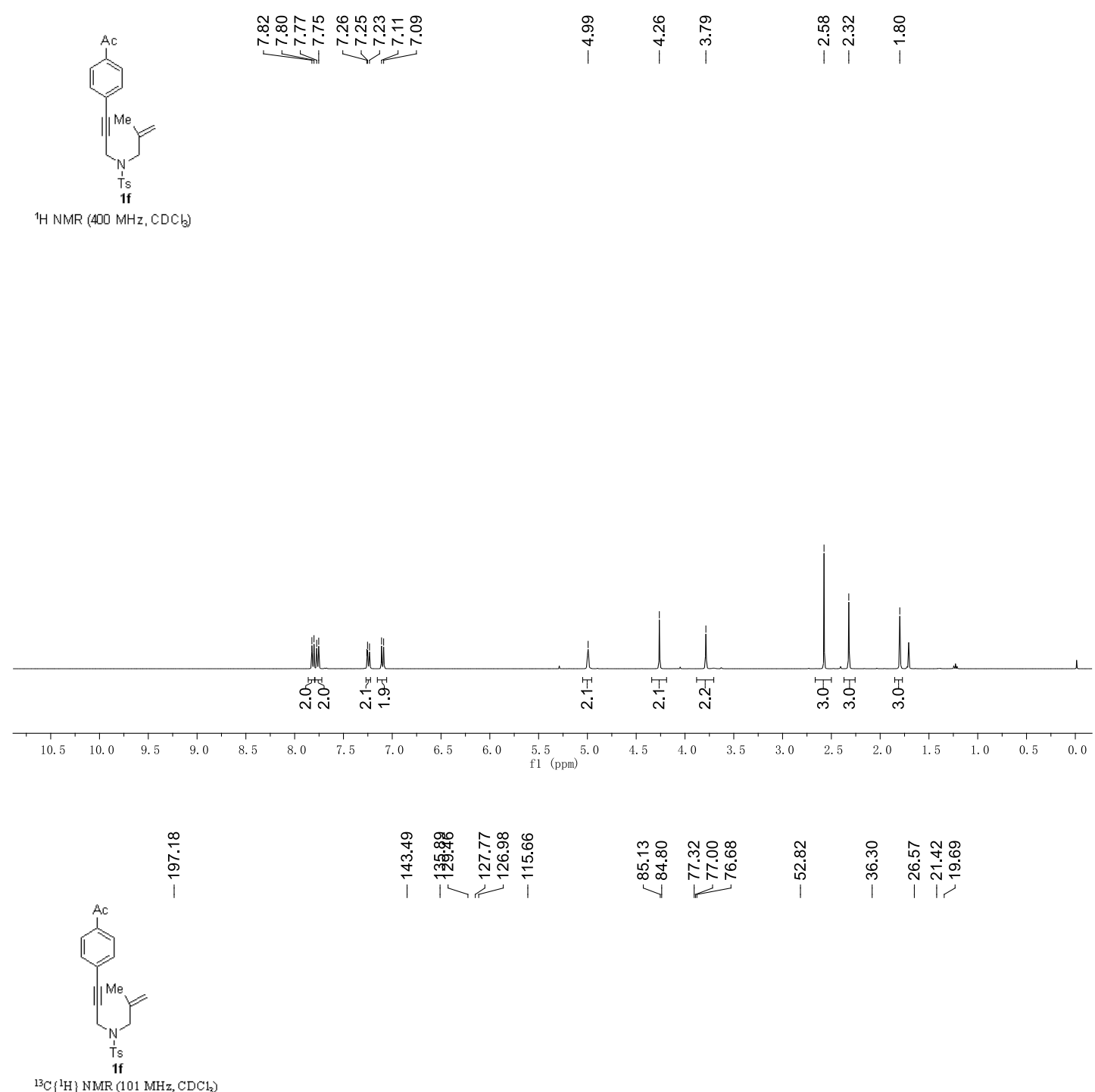

${ }^{13} \mathrm{C}\left\{{ }^{1} \mathrm{H}\right\} \operatorname{NMR}\left(101 \mathrm{MHz}, \mathrm{CDCl}_{3}\right)$

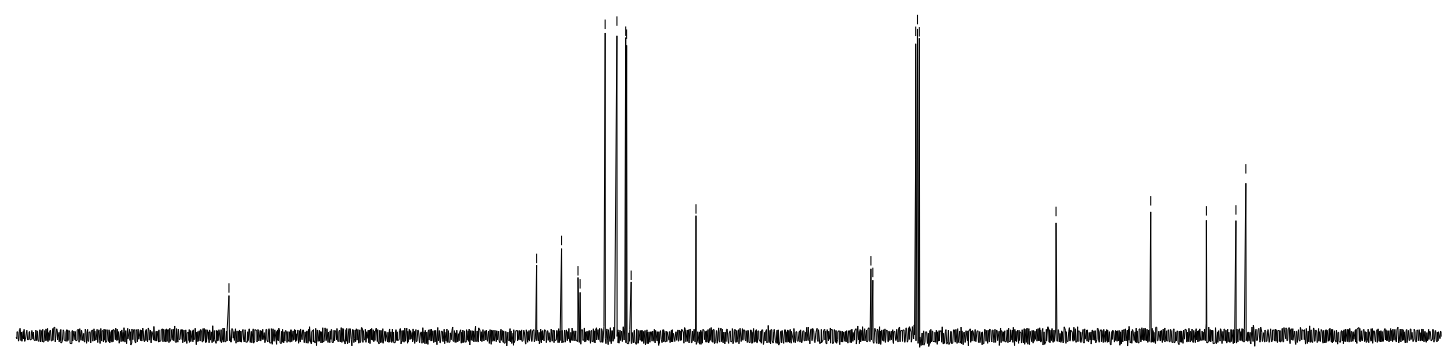

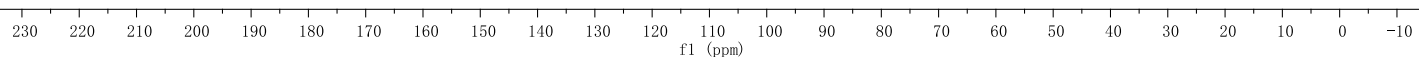




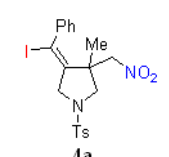

${ }^{1} \mathrm{H}$ NMR $(400 \mathrm{MHz}, \mathrm{CDC} 6)$
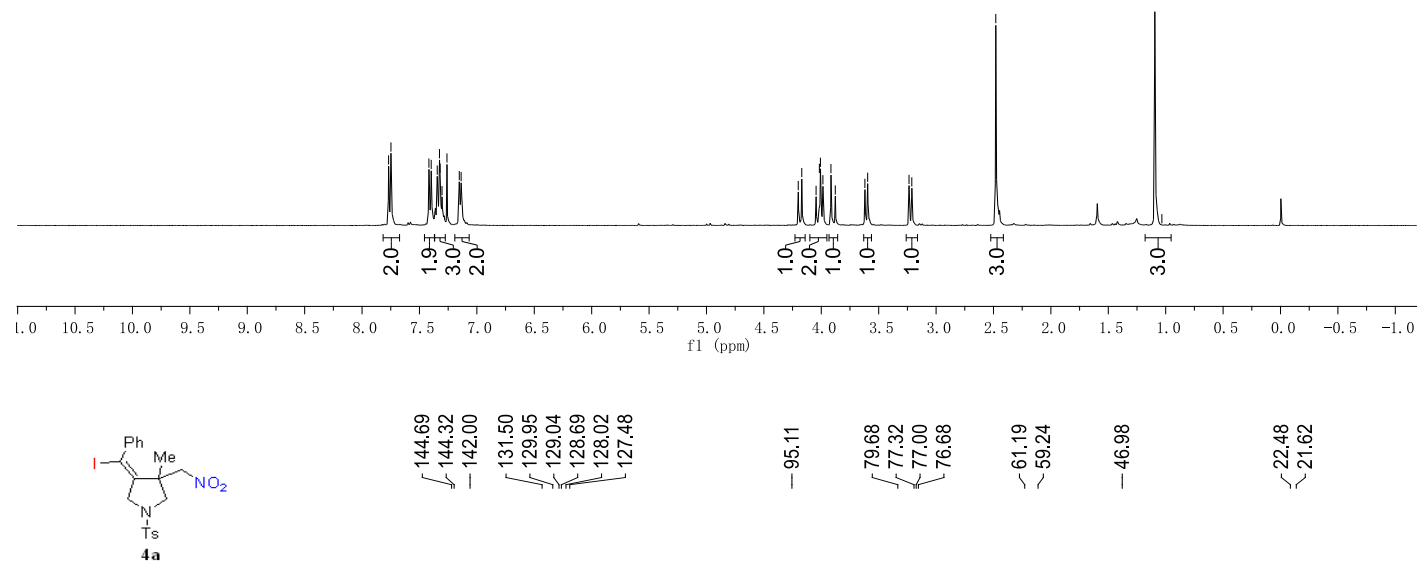

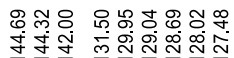

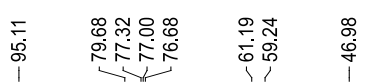

ํํำ

${ }^{13} \mathrm{C}\left\{{ }^{1} \mathrm{H}\right\} \operatorname{NMR}(101 \mathrm{MHz}, \mathrm{CDCl}\}$
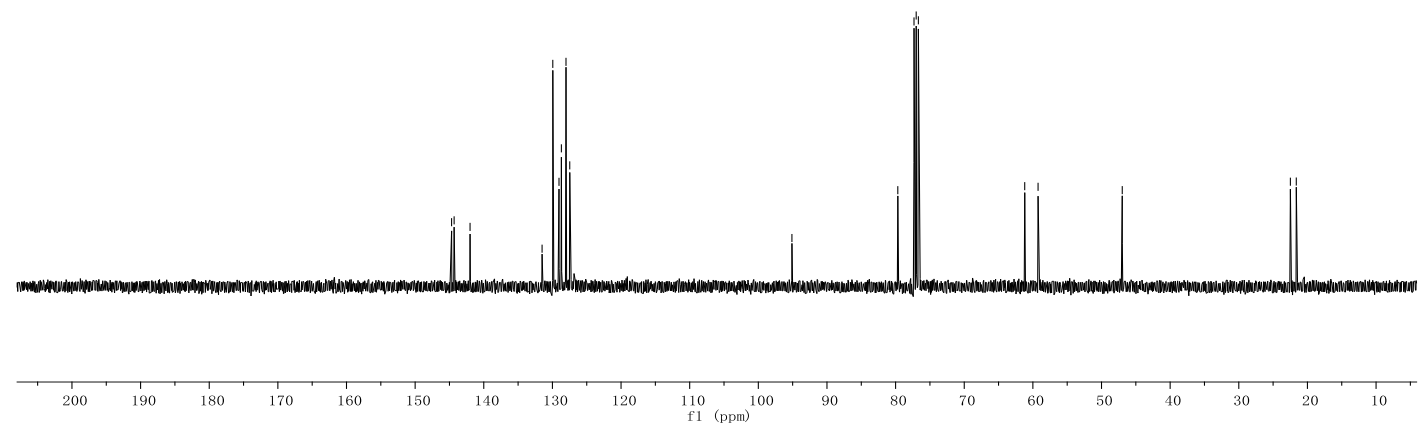

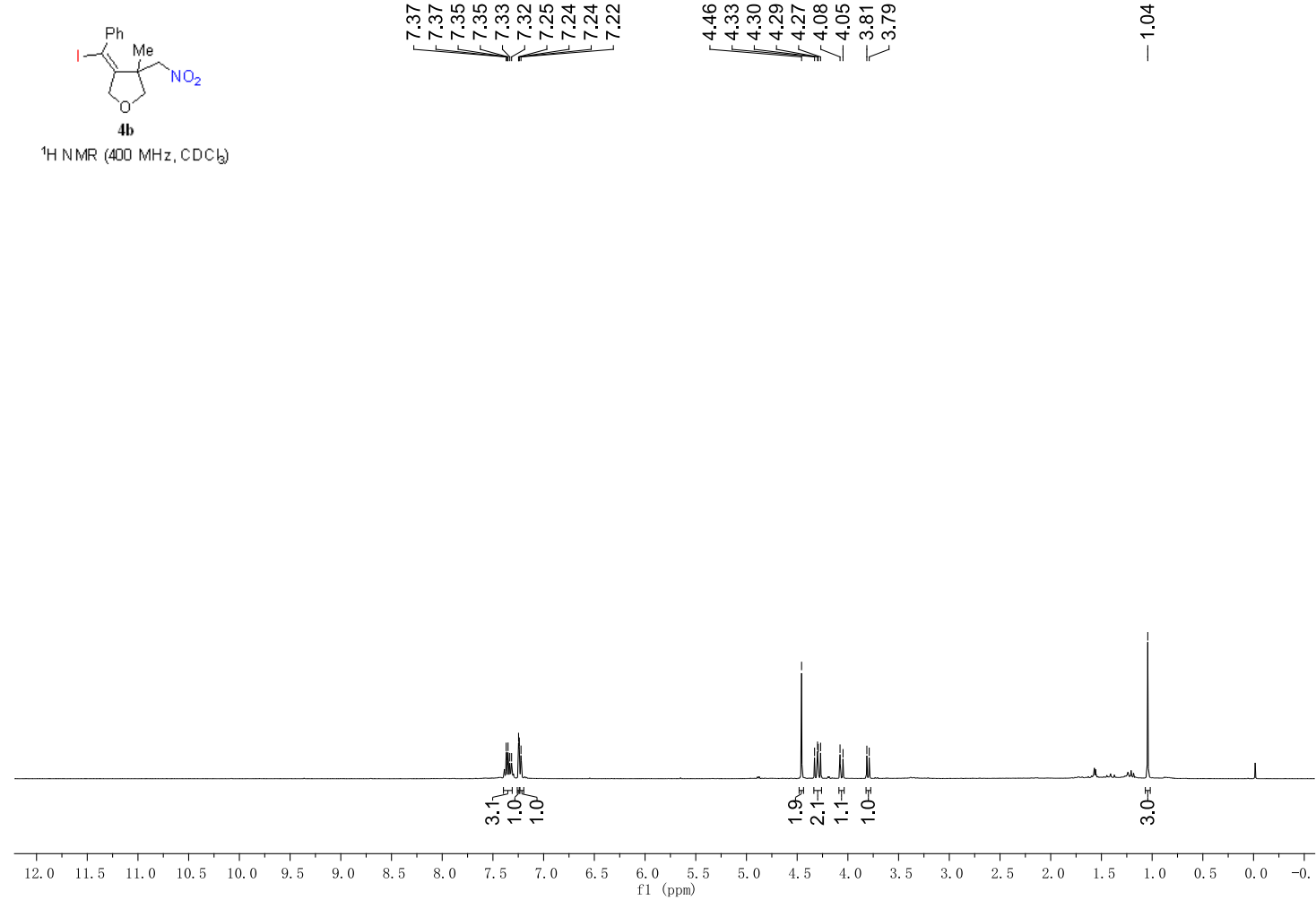

4b

${ }^{13} \mathrm{C}\left\{{ }^{1} \mathrm{H}\right\} \operatorname{NMR}\left(101 \mathrm{MHz}, \mathrm{CDCl}_{3}\right\}$

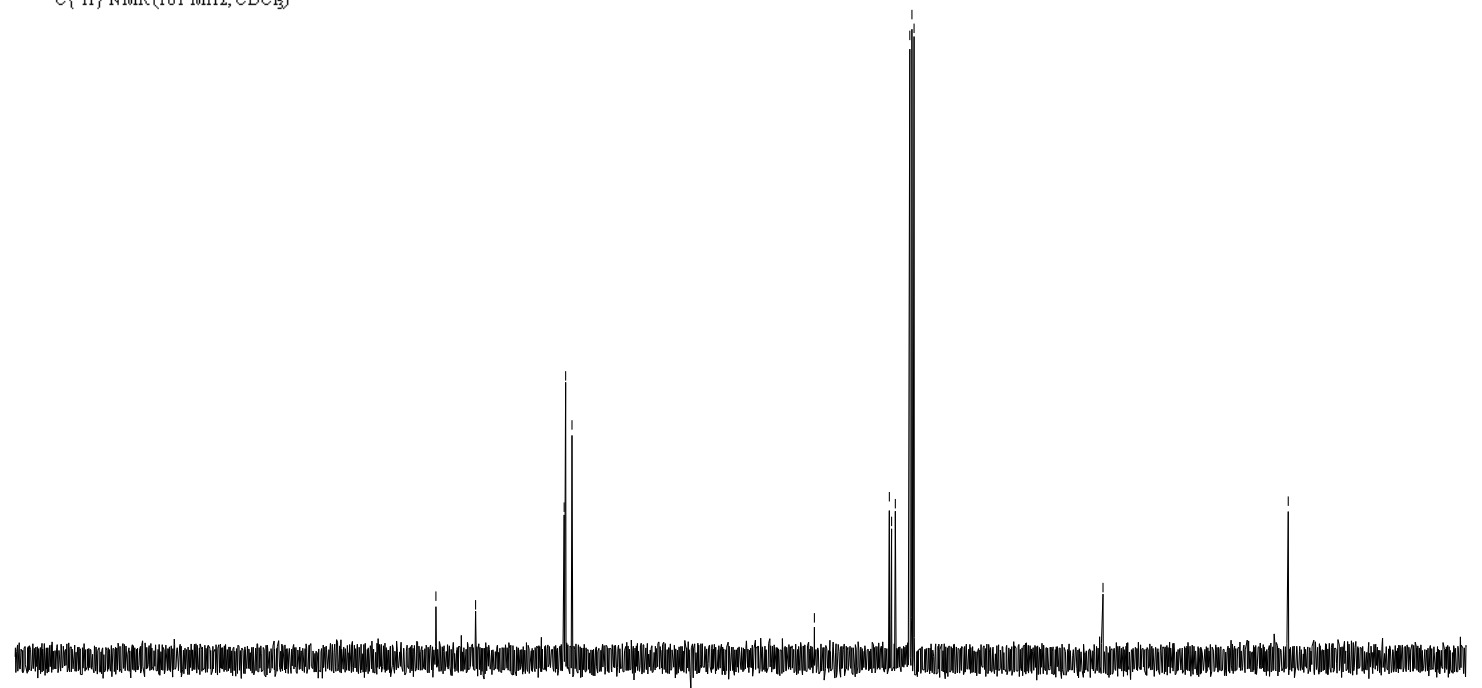

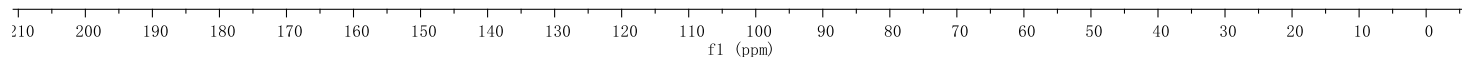


$\overbrace{\mathrm{CO}_{2} \mathrm{Me}}^{\mathrm{MnO}}$

$\stackrel{4 c}{N M R}(400 \mathrm{MHz}, \mathrm{CDC}$

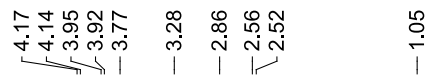

\section{$\stackrel{-1}{1}$}

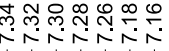




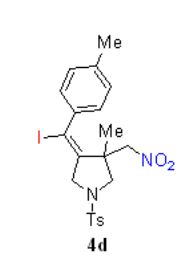

순.

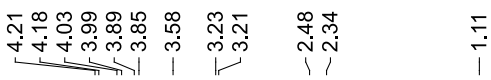

${ }^{1} \mathrm{H}$ NMR $(400 \mathrm{MHz}, \mathrm{CDCl})$

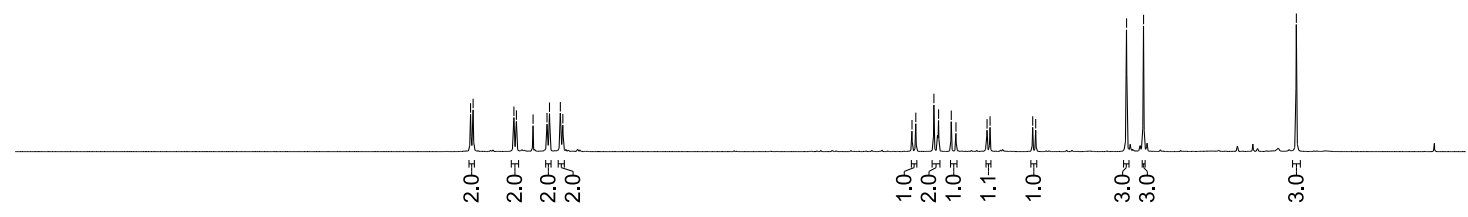

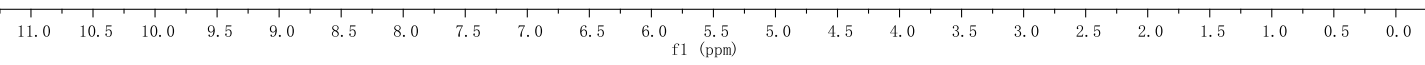

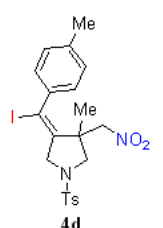

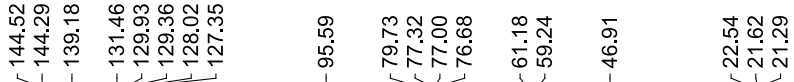

${ }^{13} \mathrm{C}\left\{{ }^{1} \mathrm{H}\right\}$ NMR $\left.(101 \mathrm{MHz}, \mathrm{CDCl})_{3}\right)$

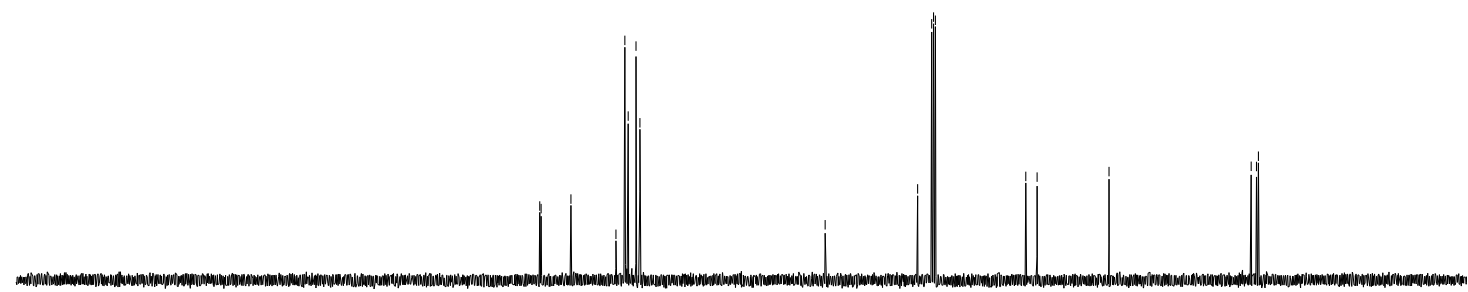

$\begin{array}{lllllllllllllllllllllllllllll}230 & 220 & 210 & 200 & 190 & 180 & 170 & 160 & 150 & 140 & 130 & 120 & 110 & 100 & 90 & 80 & 70 & 60 & 50 & 40 & 30 & 20 & 10 & 0 & -10\end{array}$ 


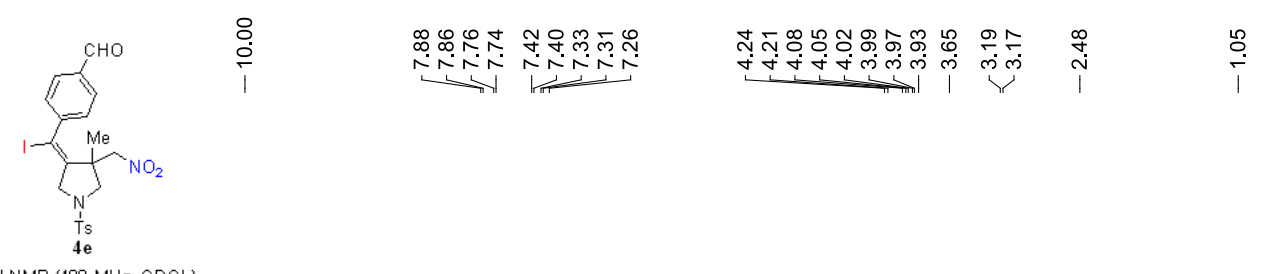

${ }^{1} \mathrm{H} N M R\left(400 \mathrm{MHz}, \mathrm{CDCl}_{3}\right)$

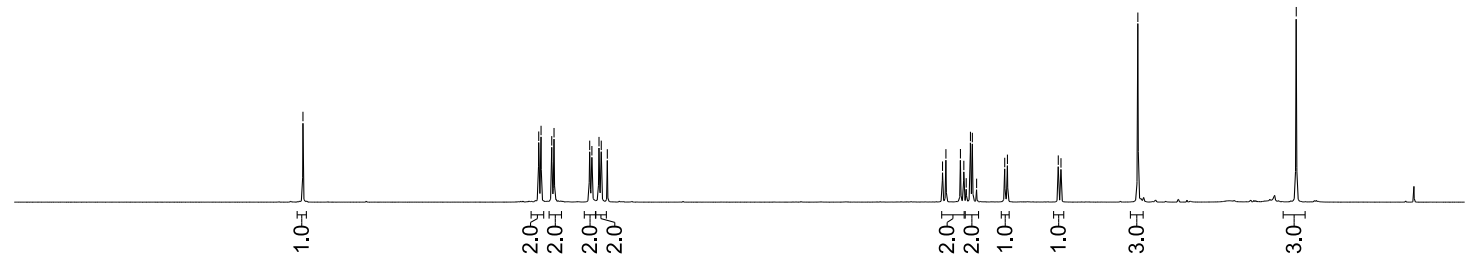

\begin{tabular}{lllllllllllllllllllllllllllll}
\hline 12.5 & 12.0 & 11.5 & 11.0 & 10.5 & 10.0 & 9.5 & 9.0 & 8.5 & 8.0 & 7.5 & 7.0 & 6.5 & 6.0 & 5.5 & 5.0 & 4.5 & 4.0 & 3.5 & 3.0 & 2.5 & 2.0 & 1.5 & 1.0 & 0.5 & 0.0
\end{tabular}

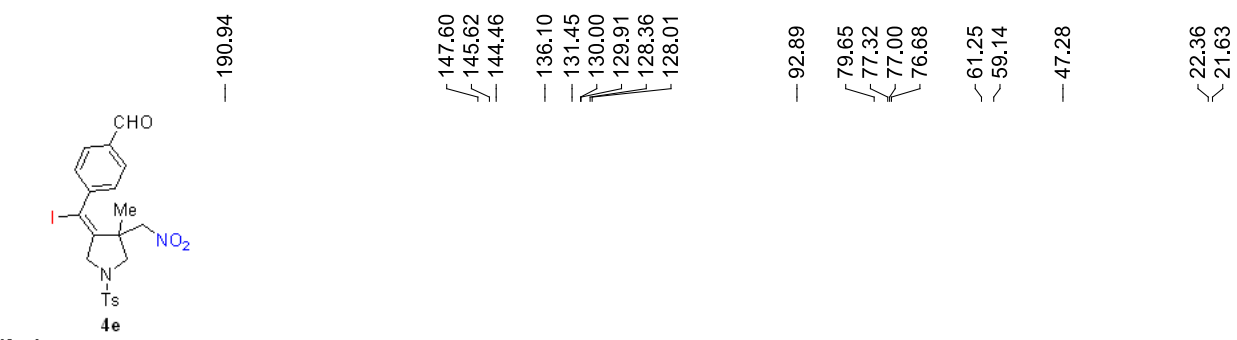

${ }^{13} \mathrm{C}\left\{{ }^{1} \mathrm{H}\right\} \operatorname{NMR}\left(101 \mathrm{MHz}, \mathrm{CDCl}_{3}\right)$

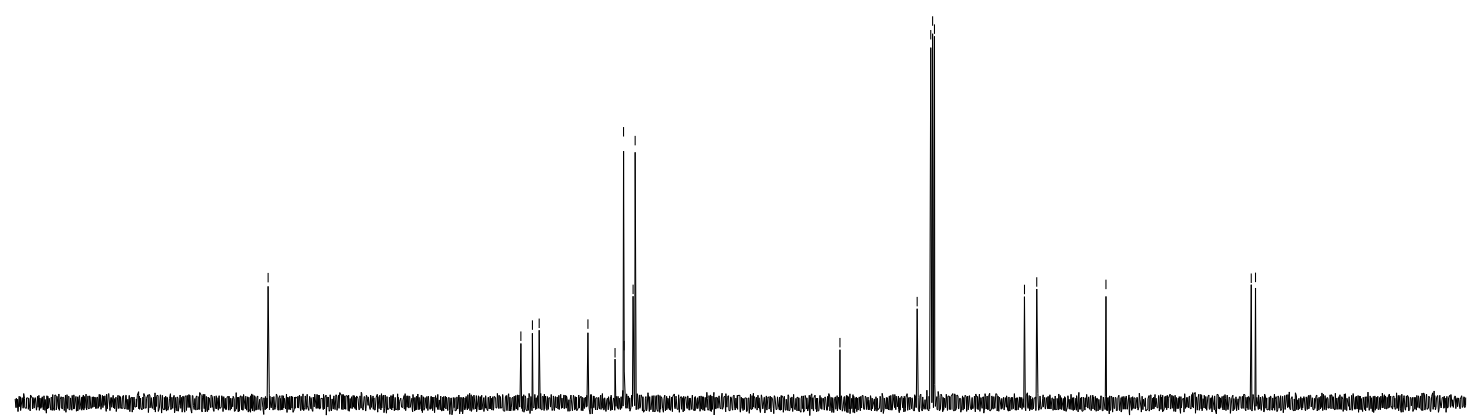

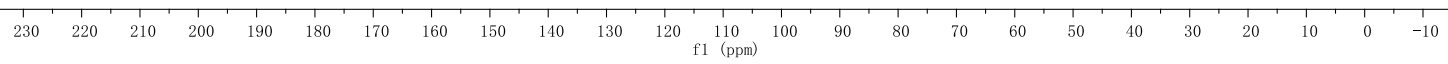




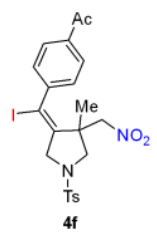

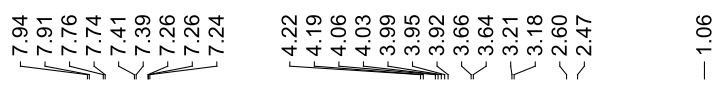

${ }^{1} \mathrm{HNMR}(400 \mathrm{MHz}, \mathrm{CDCl})$

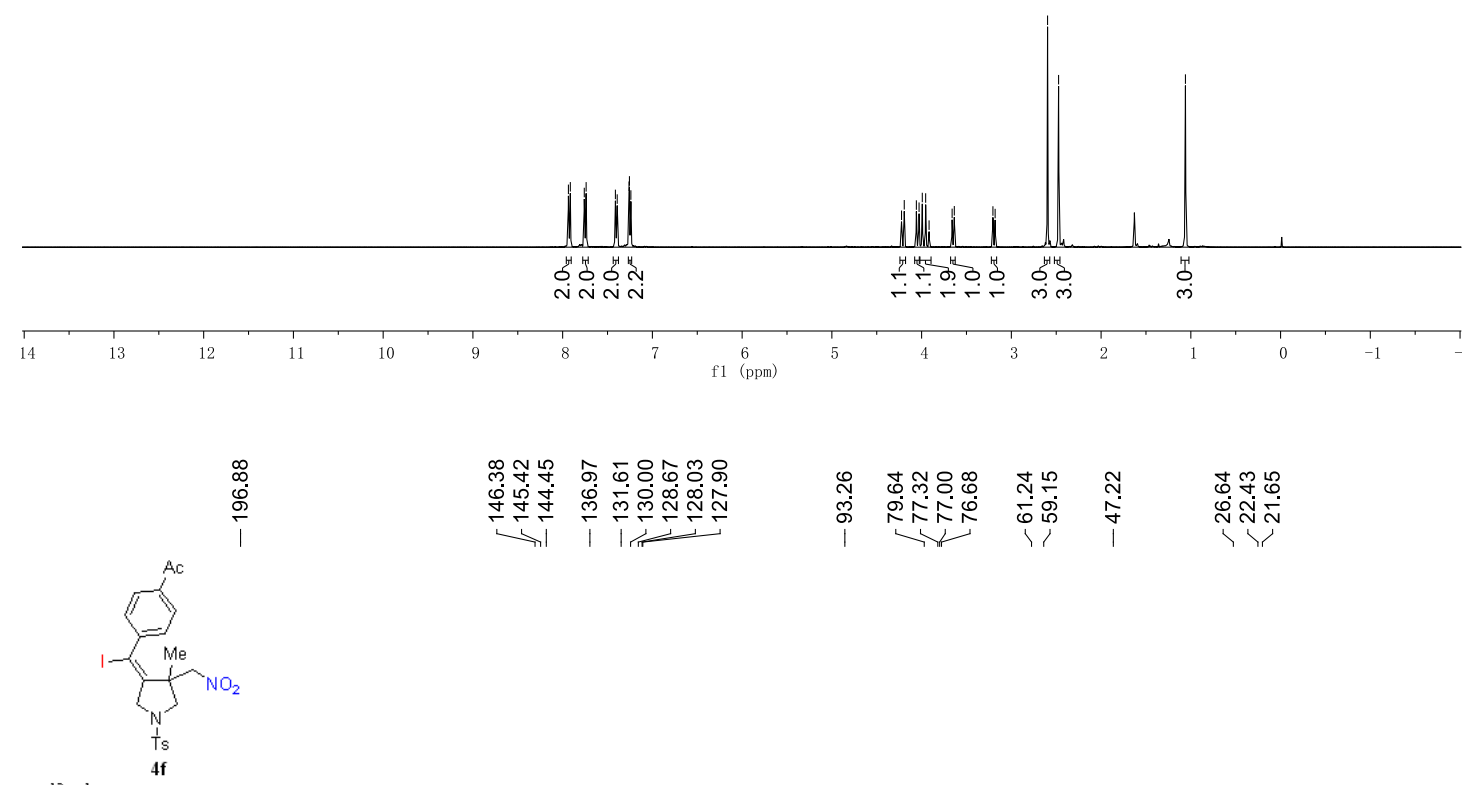

${ }^{13} \mathrm{C}\left\{{ }^{1} \mathrm{H}\right\} \operatorname{NMR}\left(101 \mathrm{MHz}, \mathrm{CDCl}_{3}\right\}$

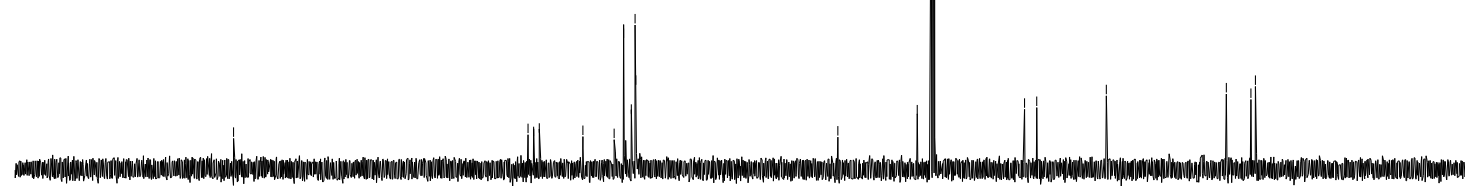

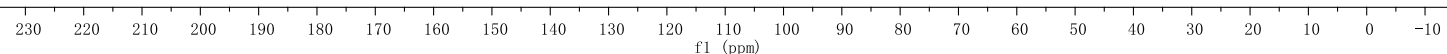




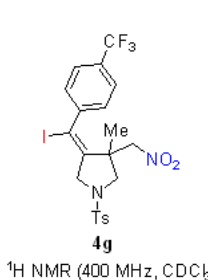

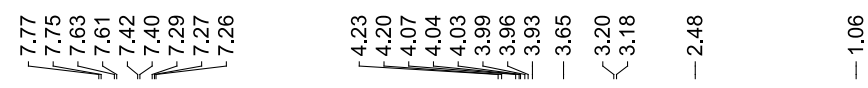

${ }^{1} \mathrm{H}$ NMR $(400 \mathrm{MHz}, \mathrm{CDCb}$

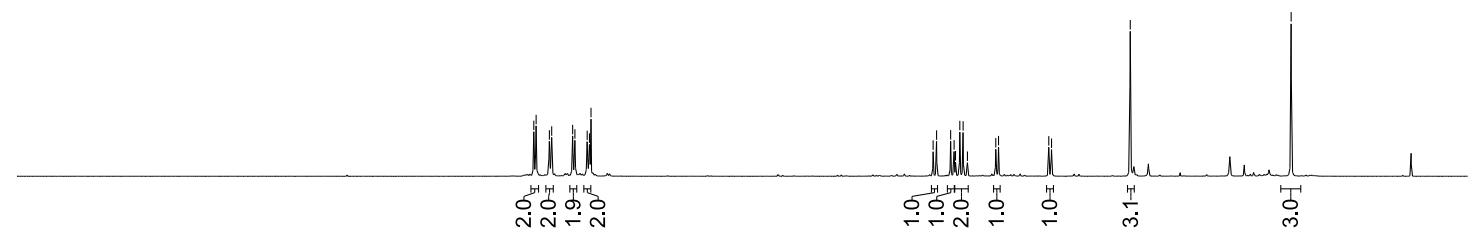

$\begin{array}{rlllllllllllllllllllllllllll}12.0 & 11.5 & 11.0 & 10.5 & 10.0 & 9.5 & 9.0 & 8.5 & 8.0 & 7.5 & 7.0 & 6.5 & \underset{f 1}{6}(\mathrm{ppm}) & 5.5 & 5.0 & 4.5 & 4.0 & 3.5 & 3.0 & 2.5 & 2.0 & 1.5 & 1.0 & 0.5 & 0.0 & -0\end{array}$

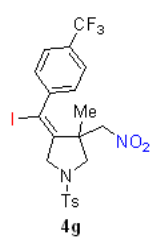

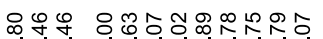

守守守

${ }^{13} \mathrm{C}\left\{{ }^{\mathrm{l}} \mathrm{H}\right\} \mathrm{NMR}\left(101 \mathrm{MHz}, \mathrm{CDCl}_{3}\right)$

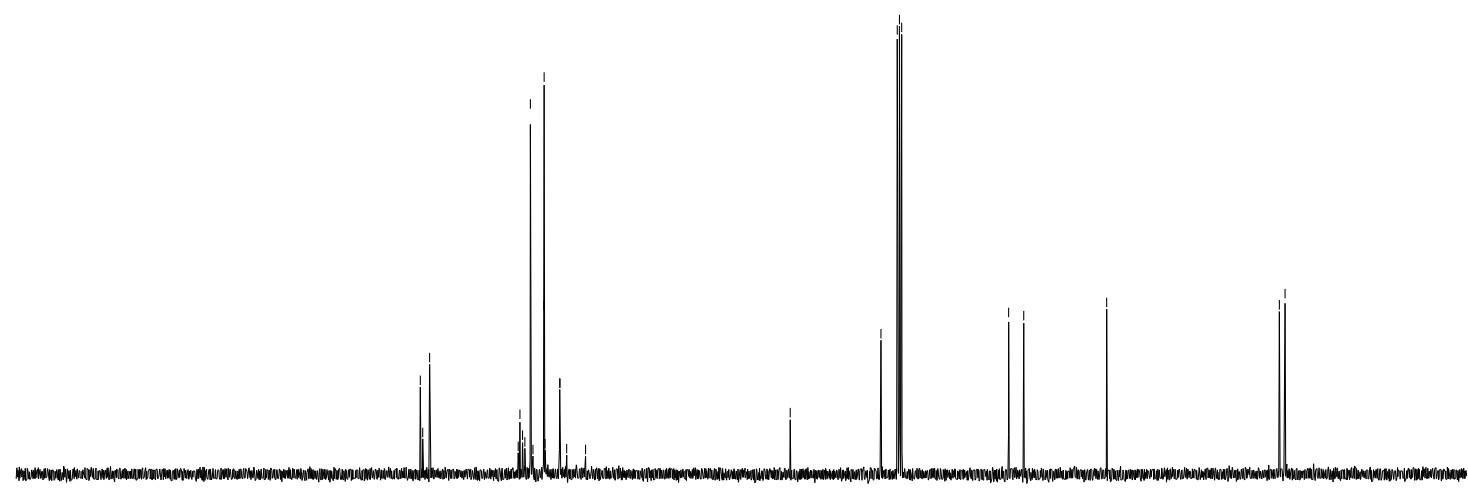

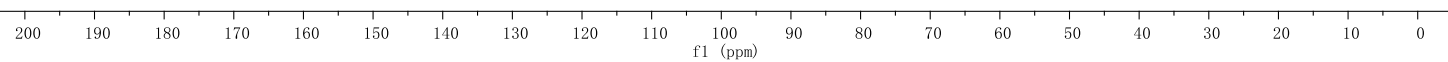




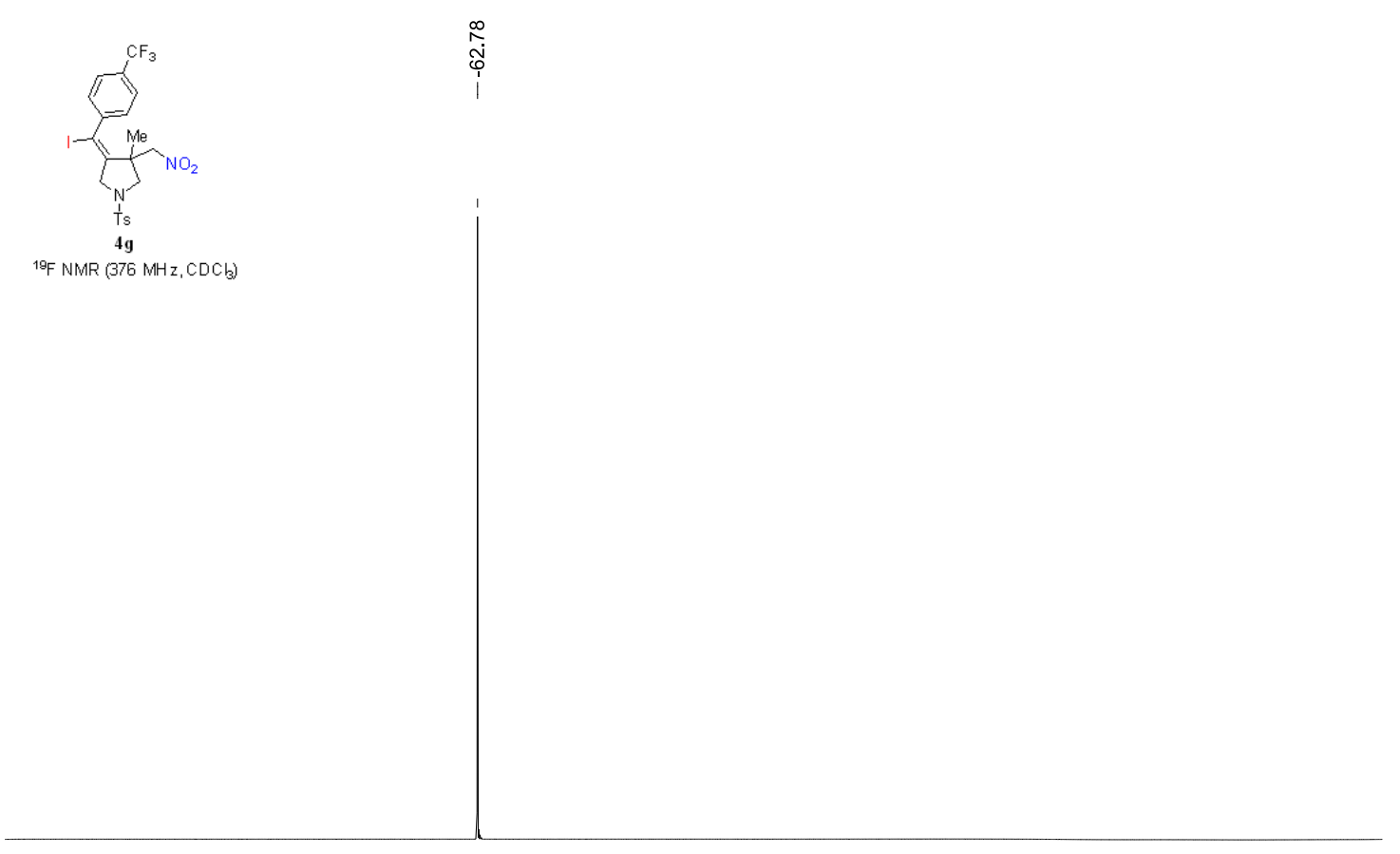

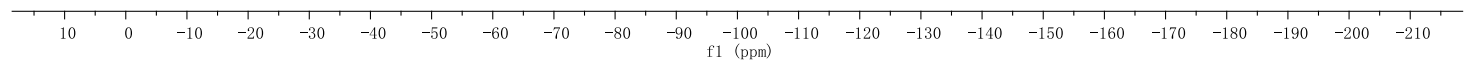

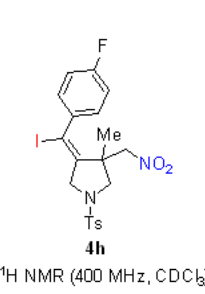

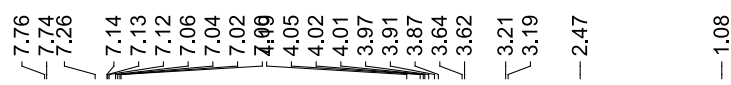

${ }^{1} \mathrm{H}$ NMR $(400 \mathrm{MHz}, \mathrm{CDCl})$

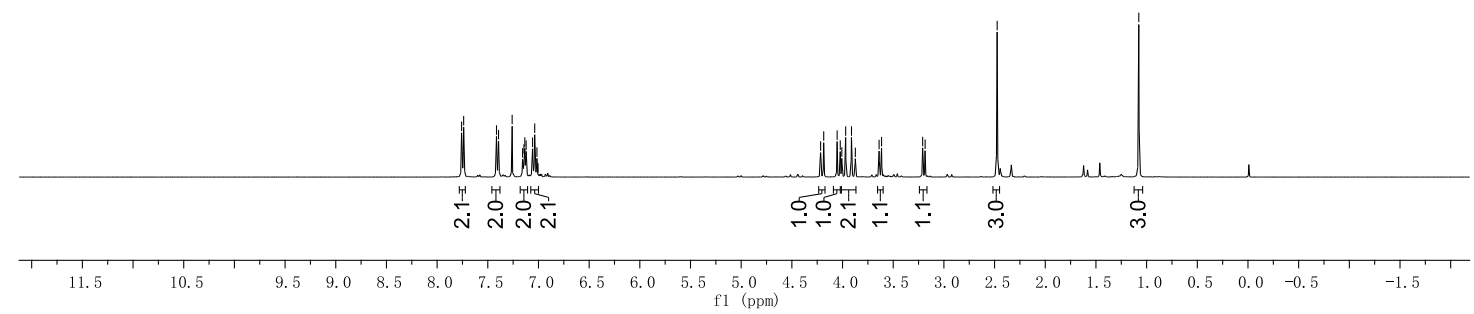




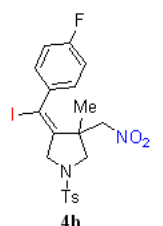

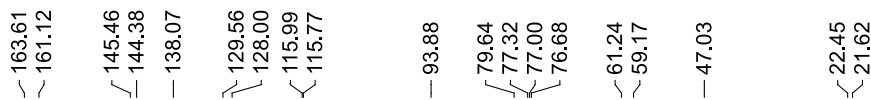

${ }^{13} \mathrm{C}\left\{{ }^{1} \mathrm{H}\right\} \operatorname{NMR}\left(101 \mathrm{MHz}, \mathrm{CDCl}_{3}\right)$
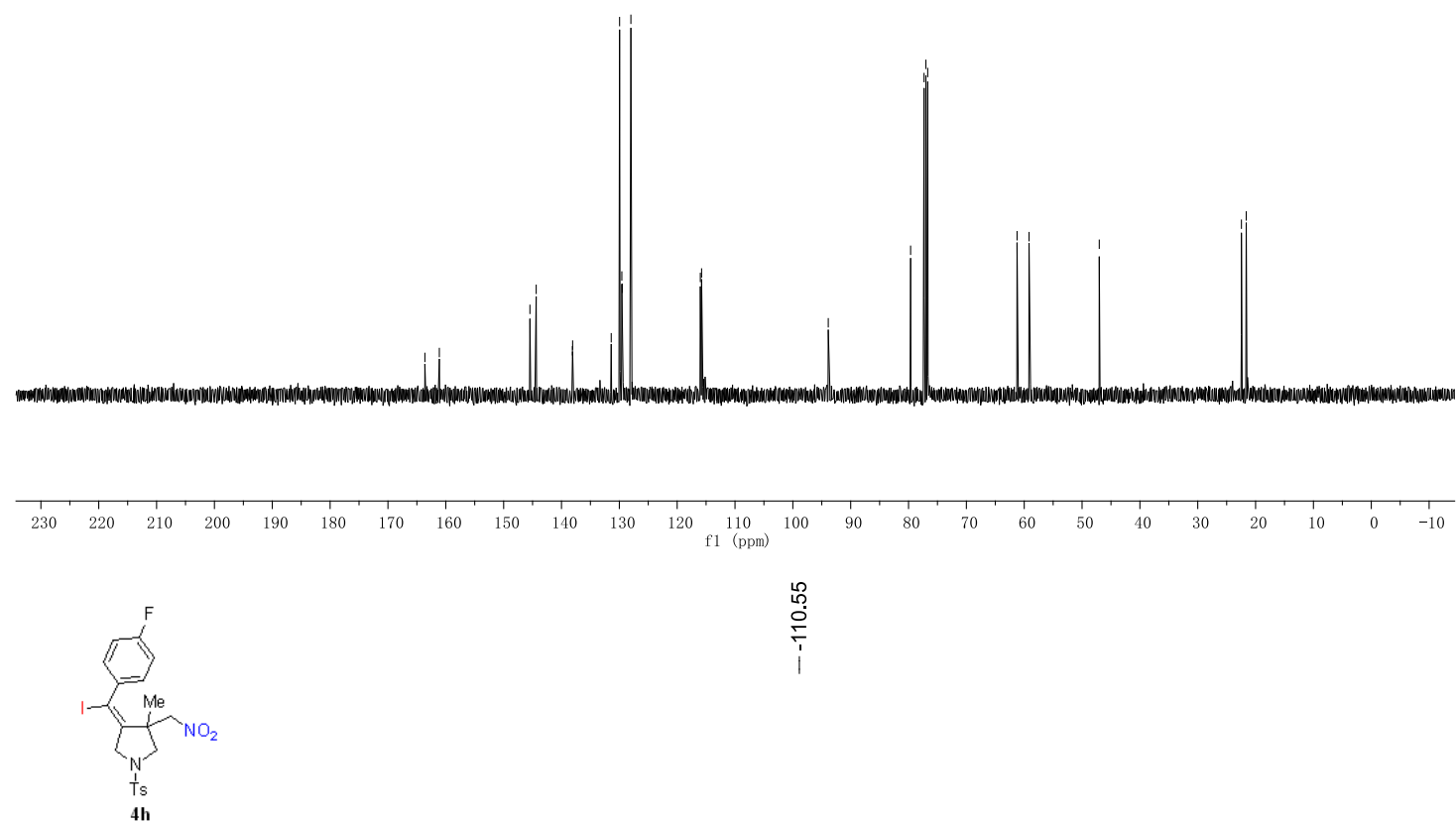

i⿱

${ }^{19} \mathrm{~F} \mathrm{NMR}$ (376 MHz, CDCb)
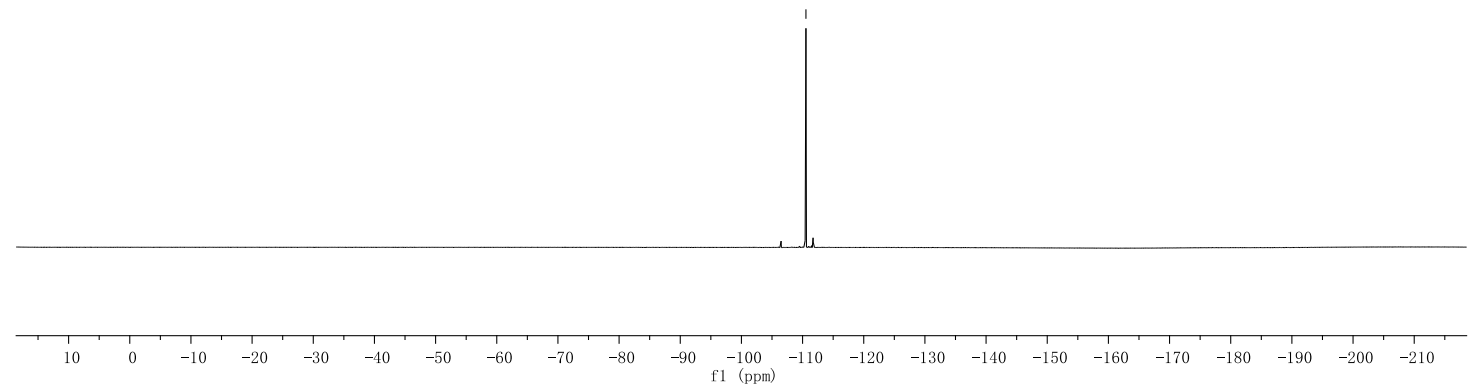


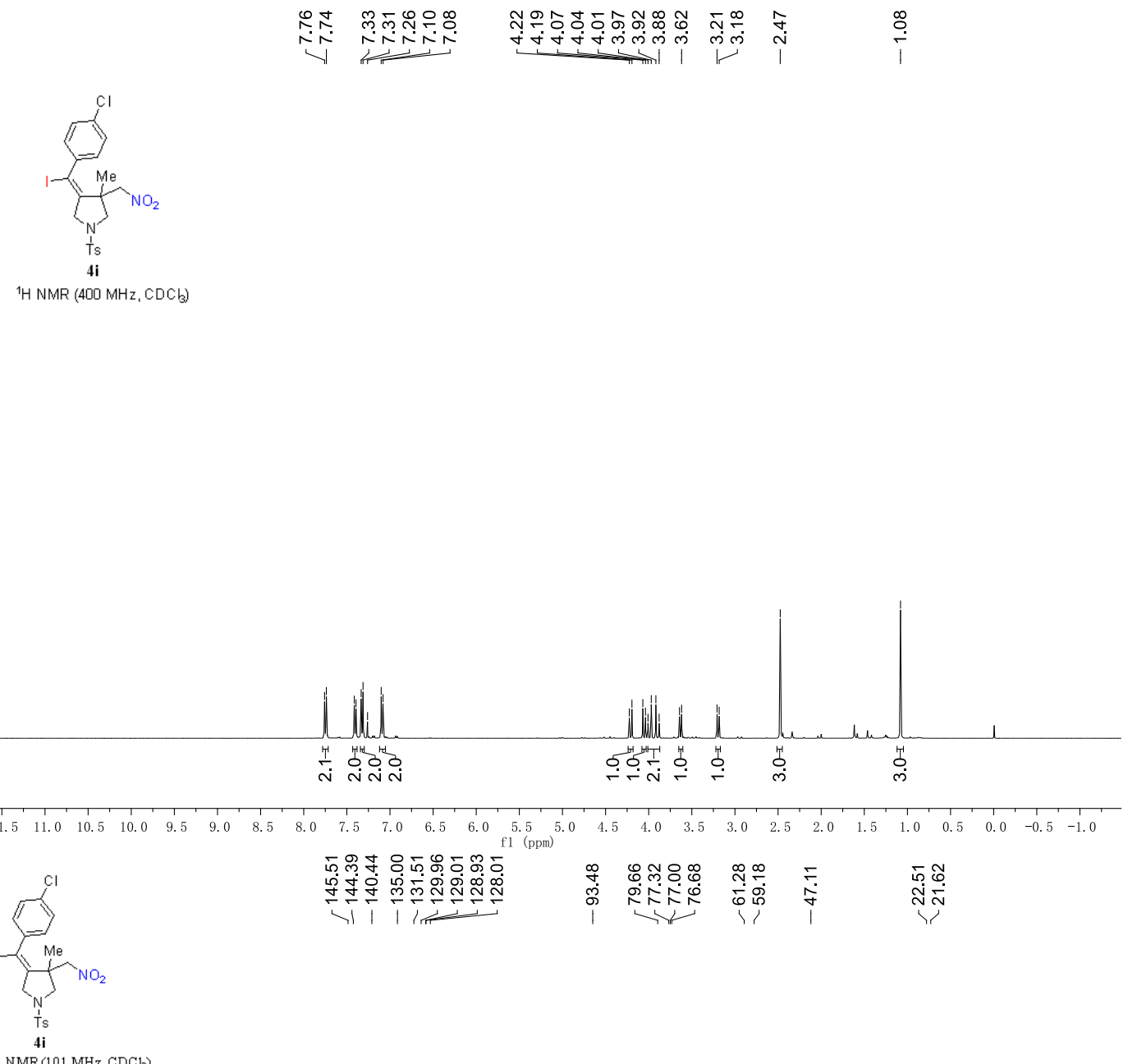

${ }^{13} \mathrm{C}\left\{{ }^{1} \mathrm{H}\right\} \operatorname{NMR}\left(101 \mathrm{MHz}, \mathrm{CDCl}_{3}\right)$

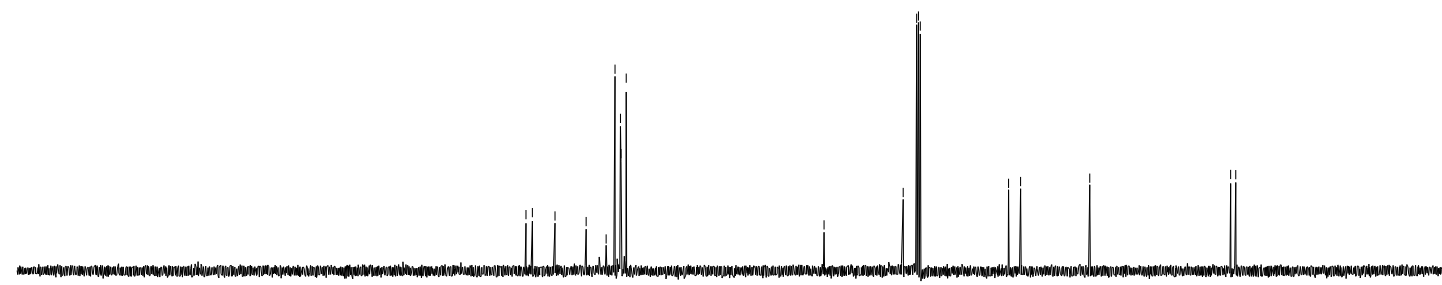

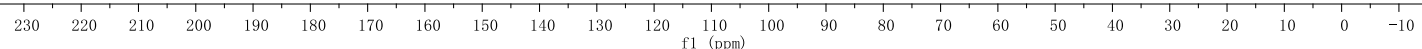




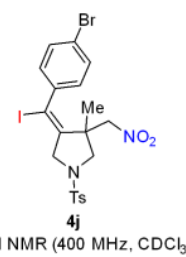

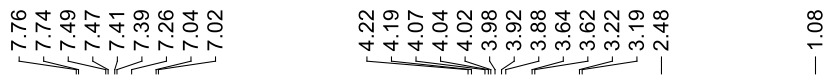

${ }^{1} \mathrm{HNMR}(400 \mathrm{MHz}, \mathrm{CDCl})$
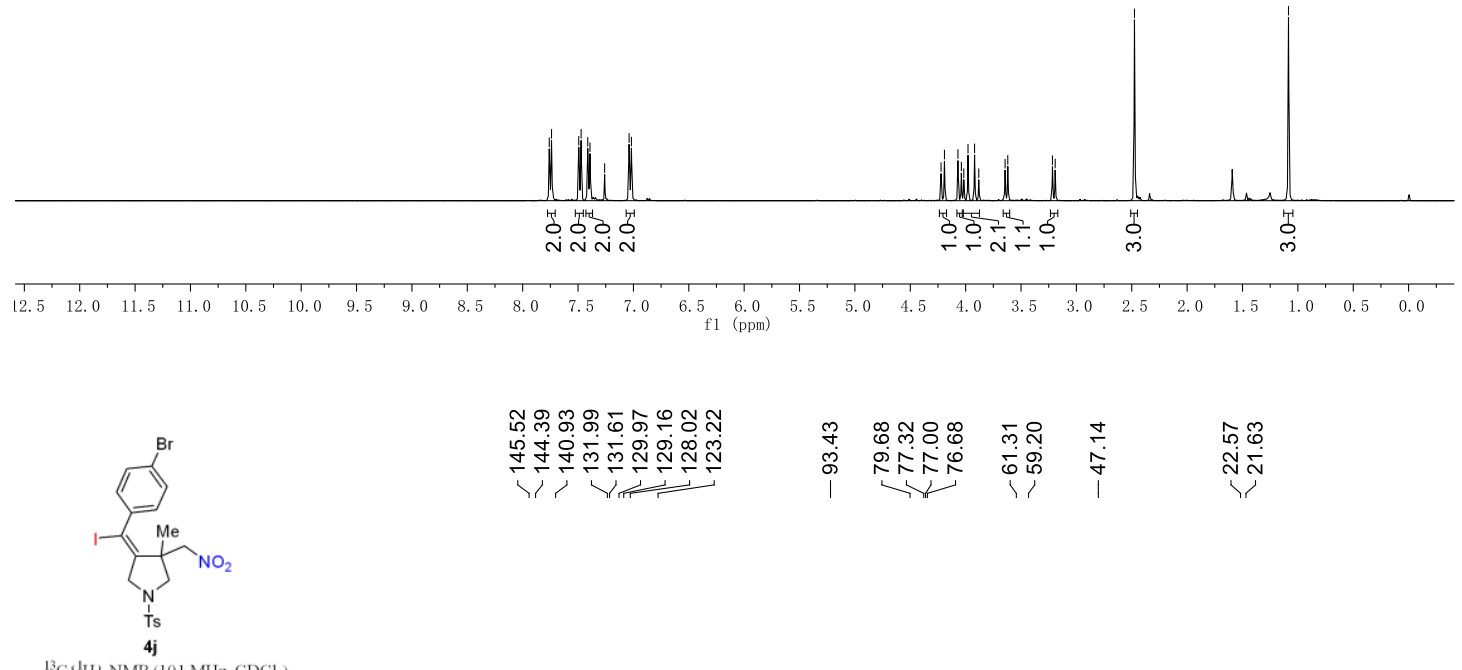

${ }^{13} \mathrm{C}\left\{{ }^{1} \mathrm{H}\right\} \mathrm{NMR}\left(101 \mathrm{MHz}, \mathrm{CDCl}_{3}\right)$

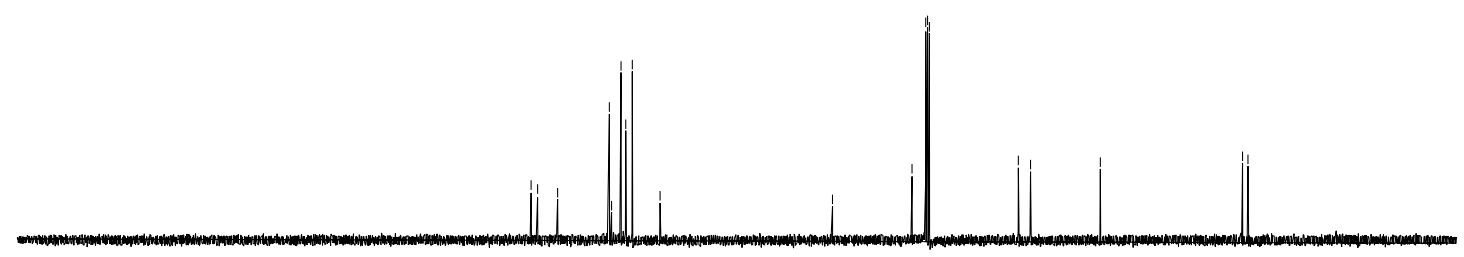

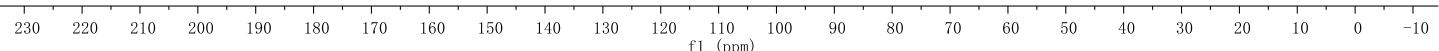




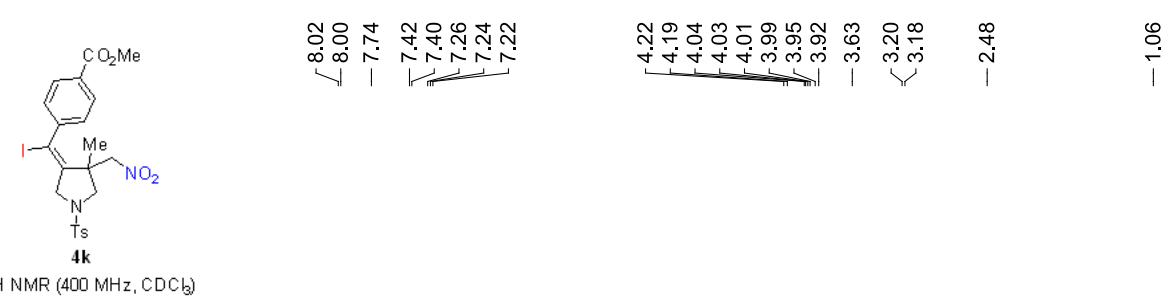

${ }^{1} \mathrm{H}$ NMR $(400 \mathrm{MHz}, \mathrm{CDCl})$
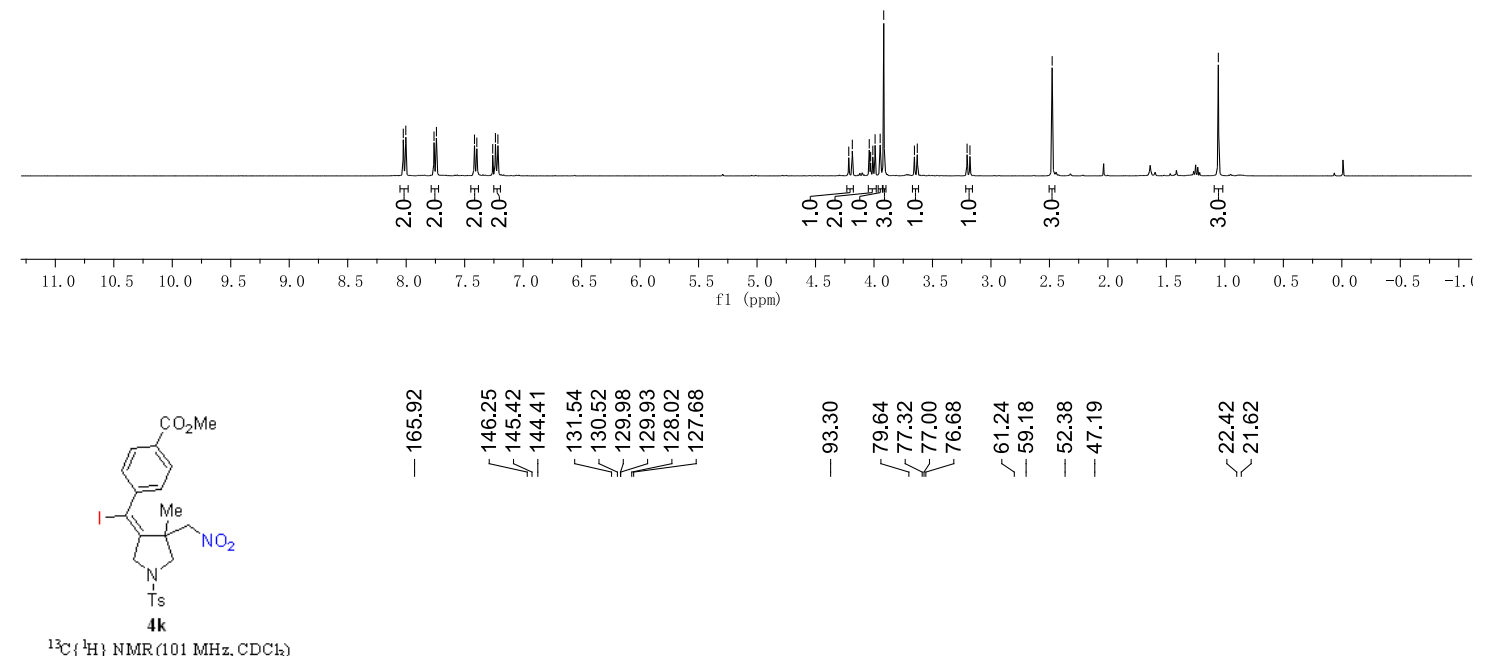

${ }^{13} \mathrm{C}\left\{{ }^{1} \mathrm{H}\right\} \operatorname{MMR}\left(101 \mathrm{MHz}, \mathrm{CDCl} 1_{3}\right)$

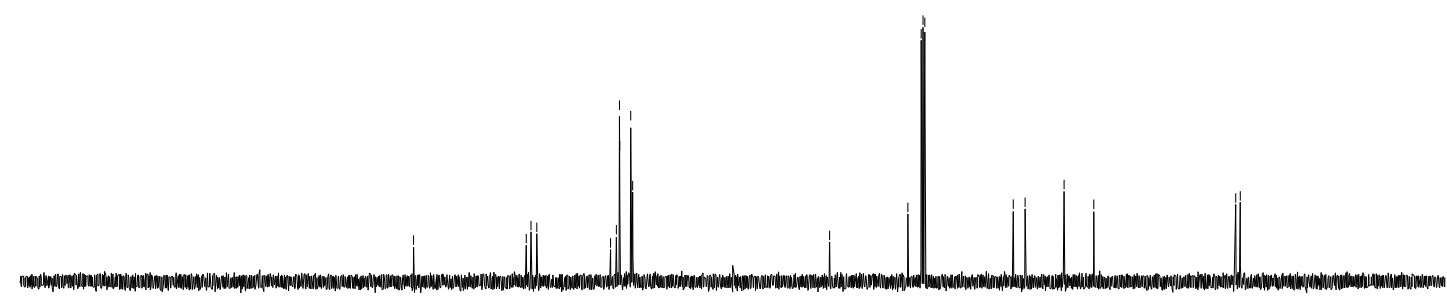

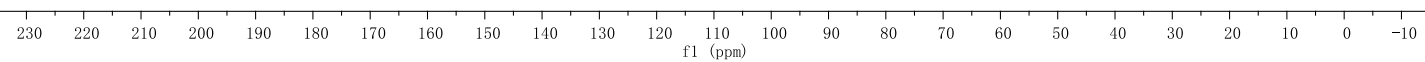




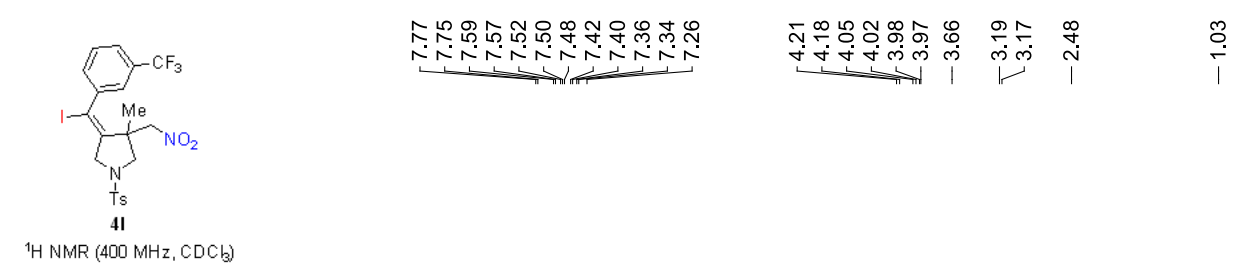

${ }^{1} \mathrm{H}$ NMR $(400 \mathrm{MHz}, \mathrm{CDCl})$
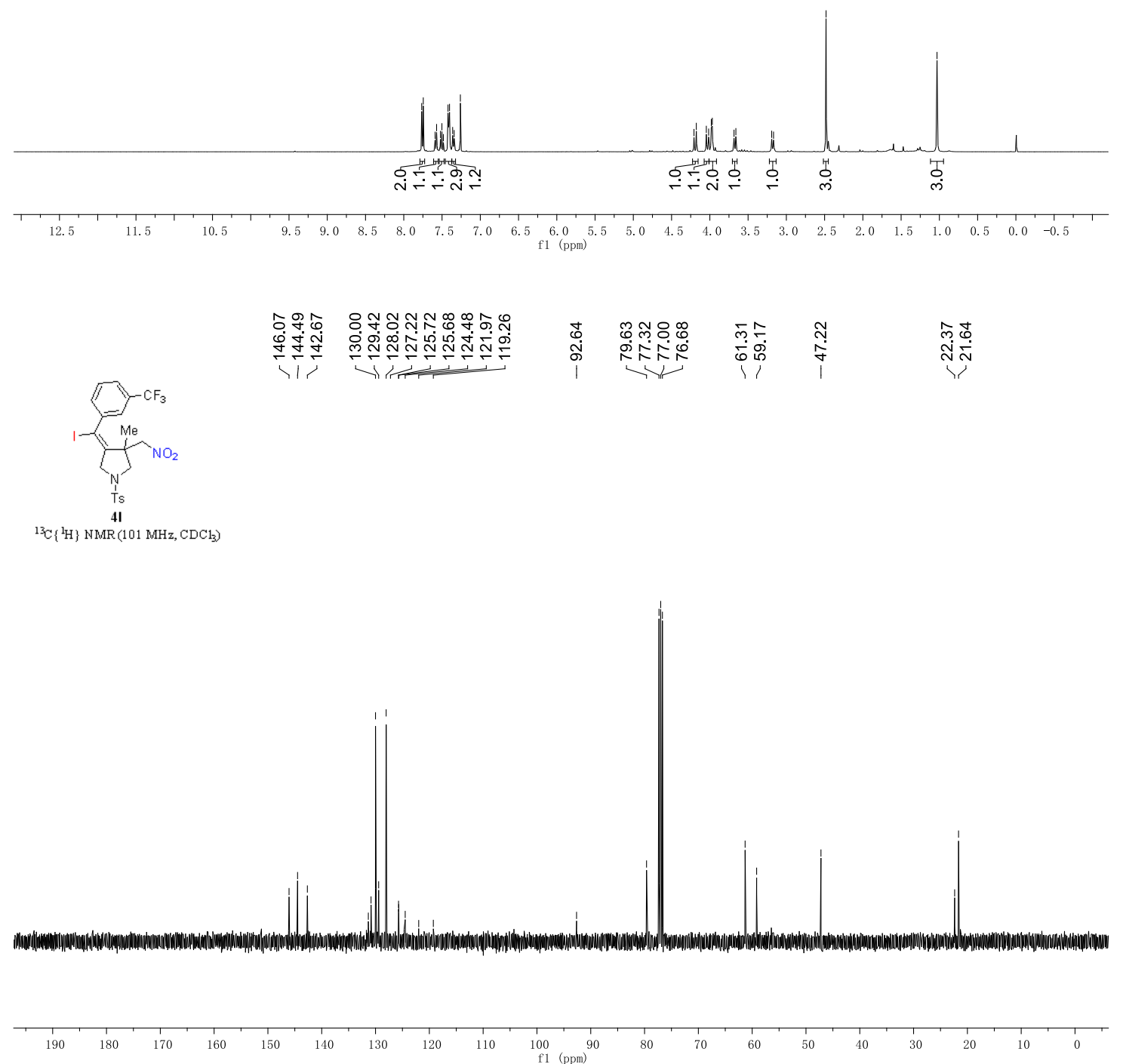

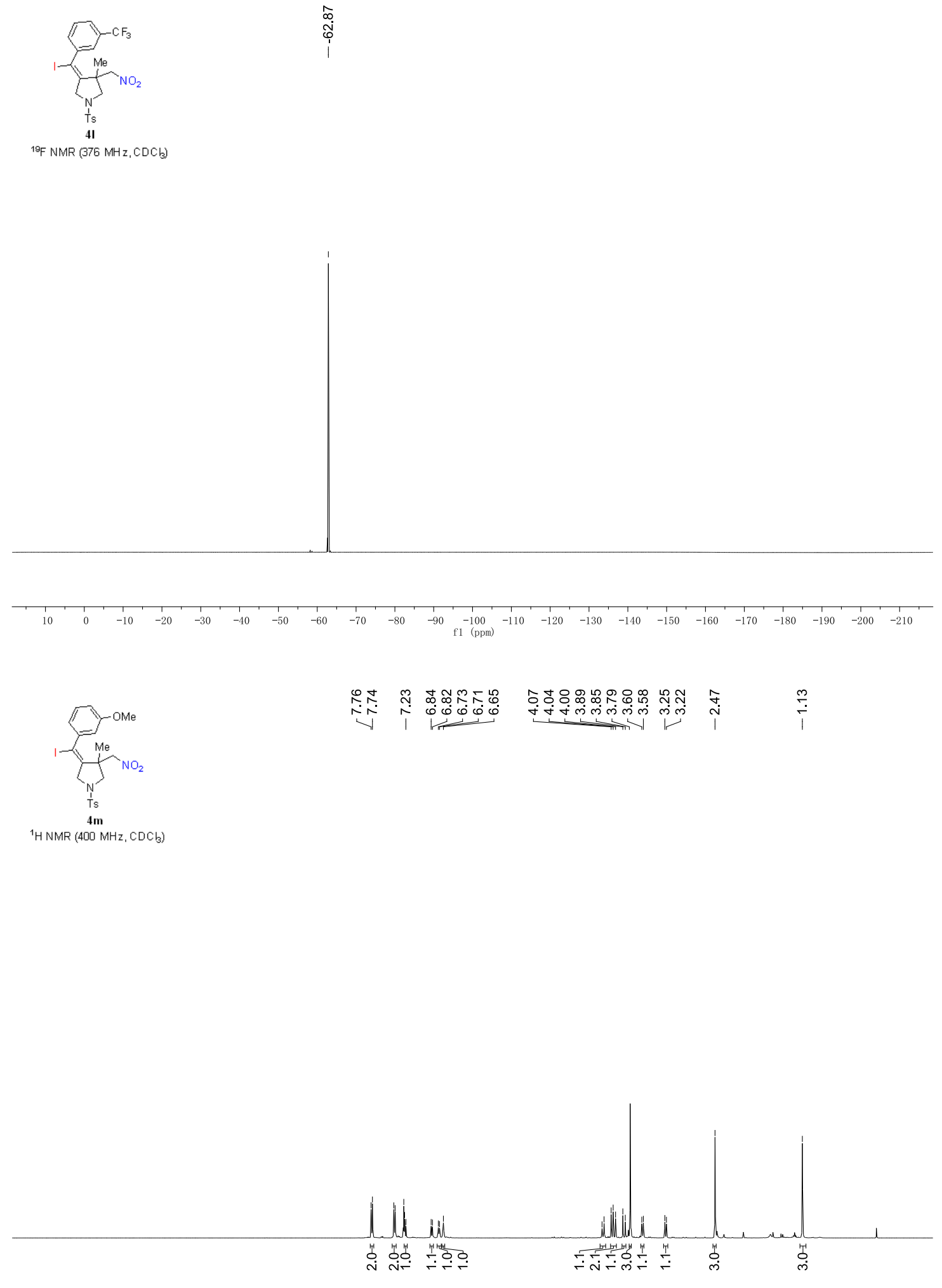

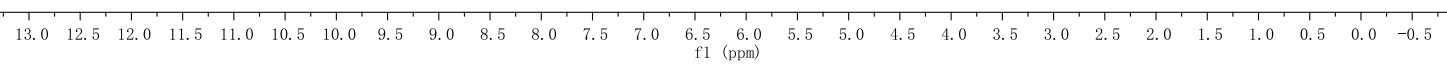



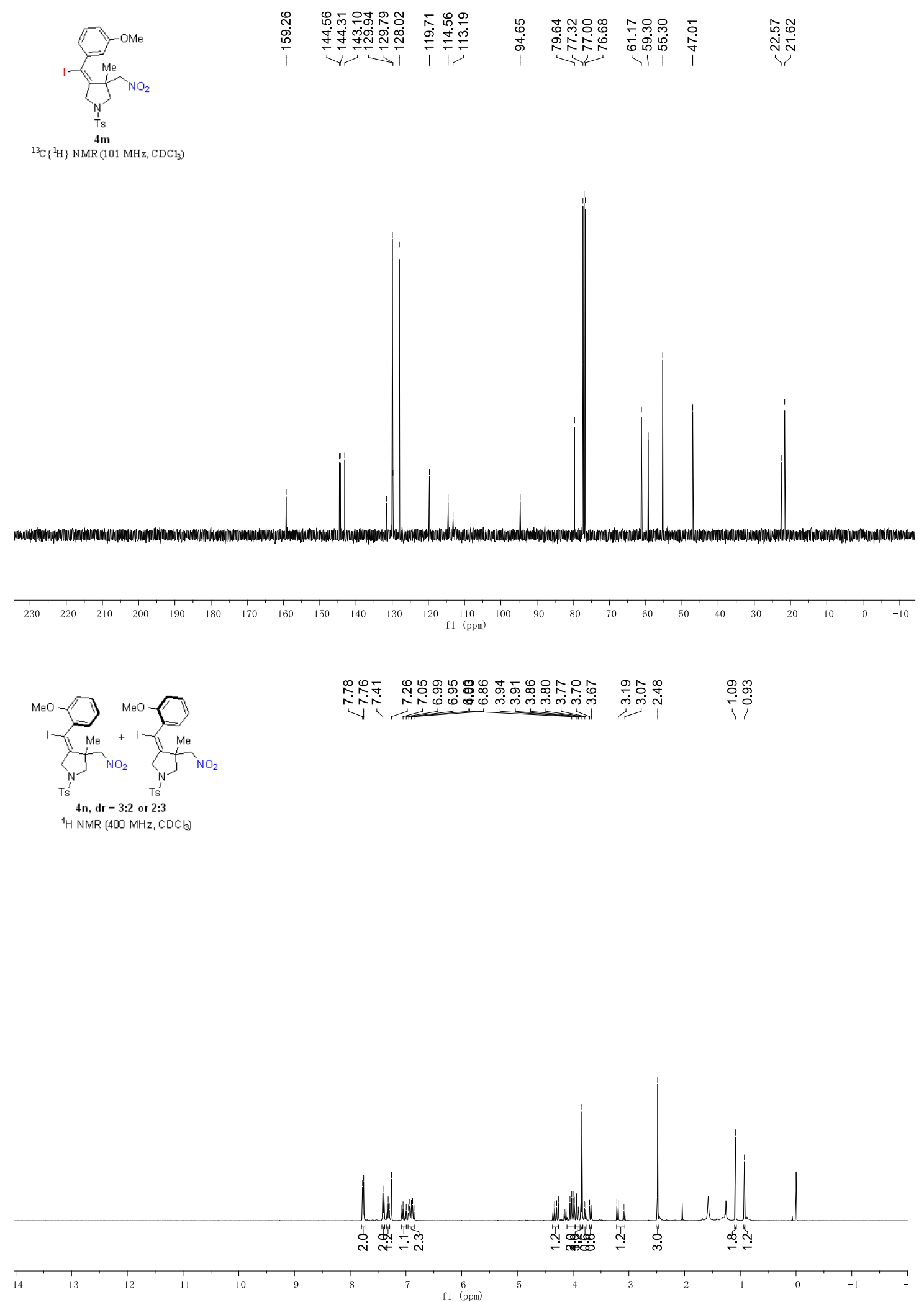


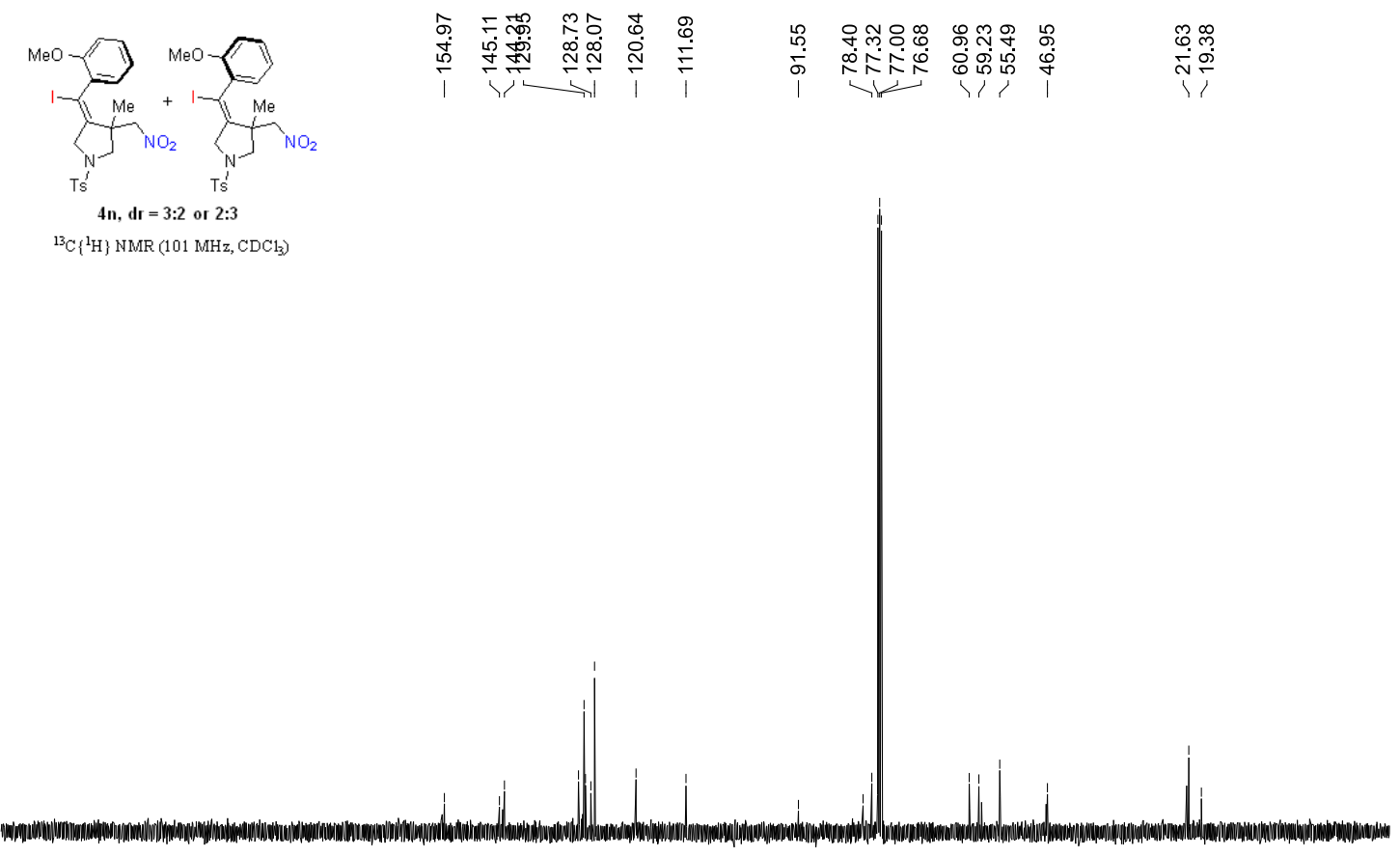

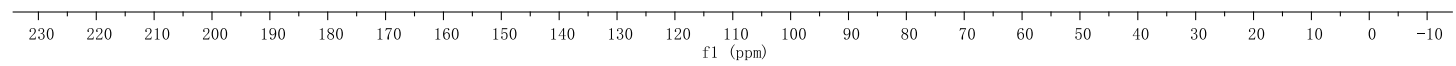

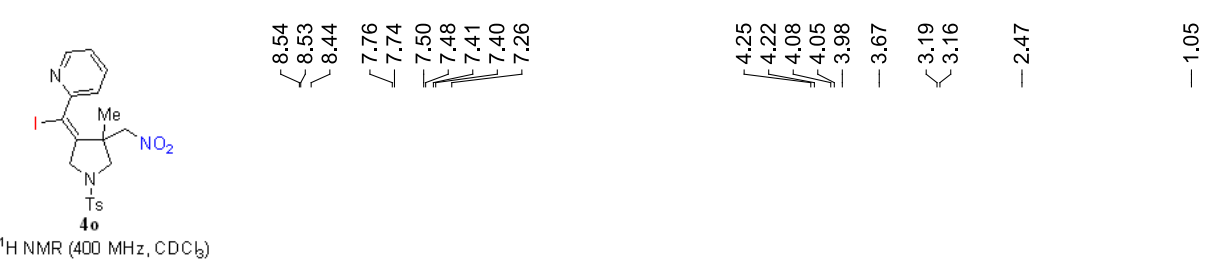

${ }^{1} \mathrm{HNMR}(400 \mathrm{MHz}, \mathrm{CDCl})$

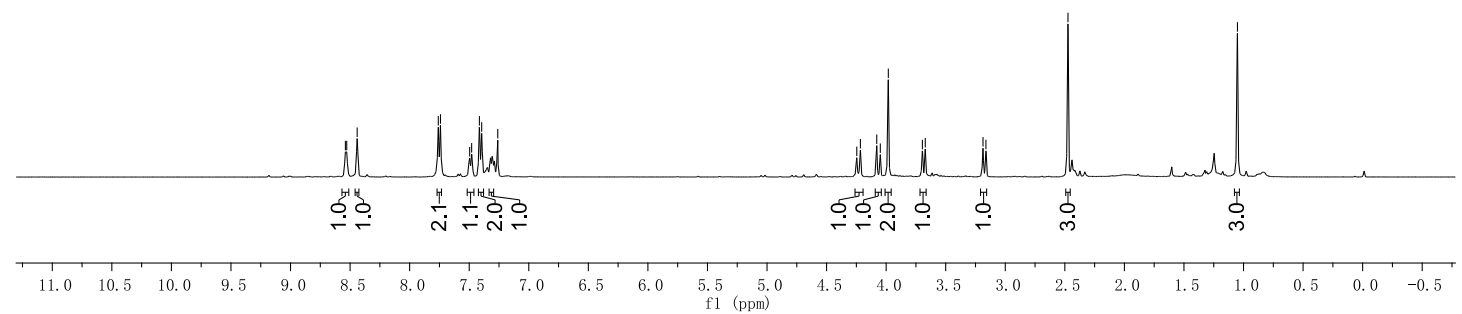




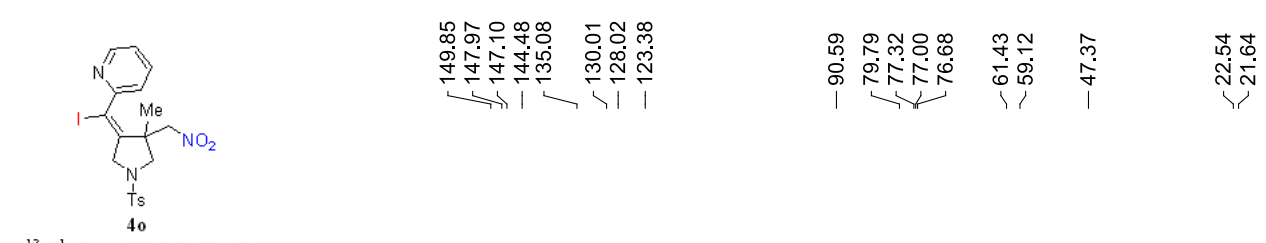

${ }^{13} \mathrm{C}\left\{{ }^{1} \mathrm{H}\right\} \operatorname{NMR}\left(101 \mathrm{MHz}, \mathrm{CDCl}_{3}\right)$
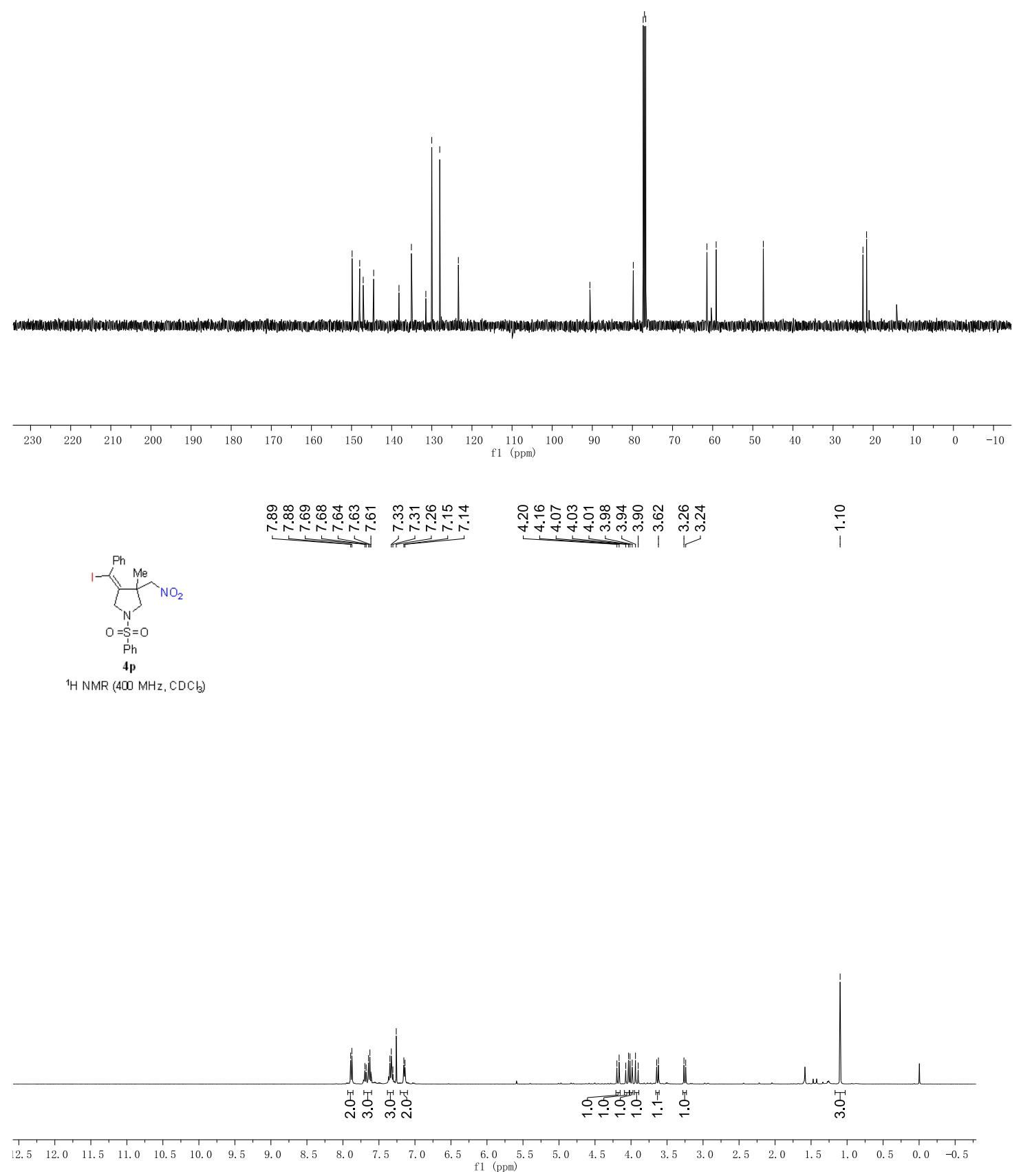


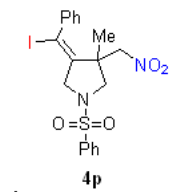

li

$\left.{ }^{13} \mathrm{C}_{\{}{ }^{1} \mathrm{H}\right\} \operatorname{MMR}\left(101 \mathrm{MHz}, \mathrm{CDCl}_{3}\right)$
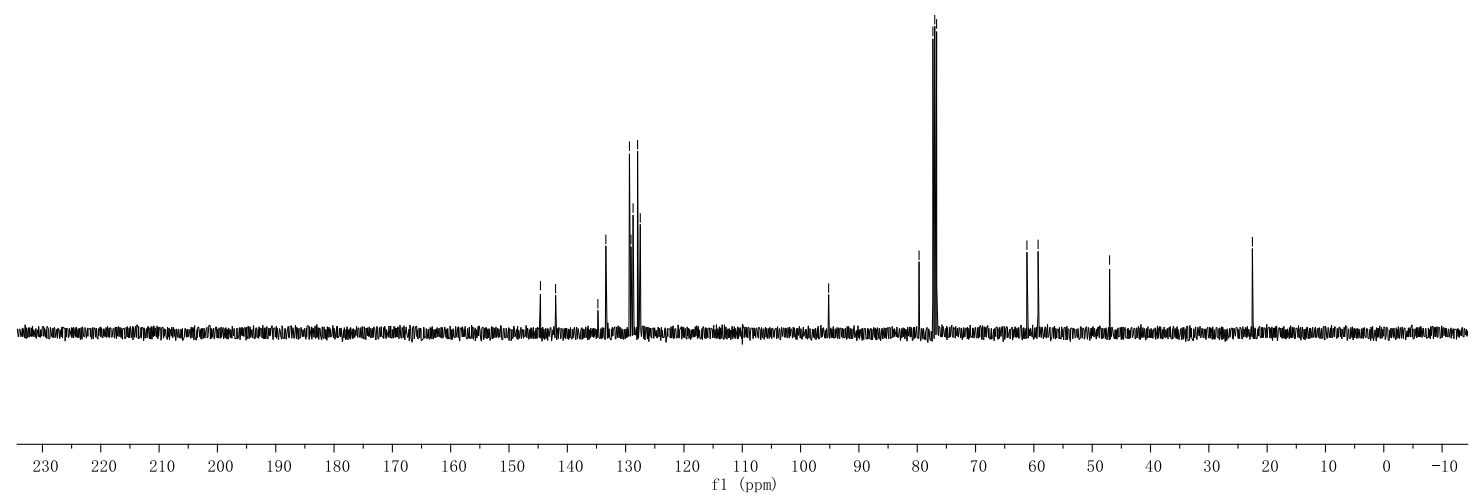

可 0

$\stackrel{0}{i}$

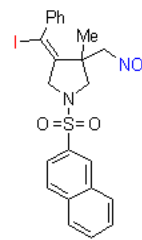

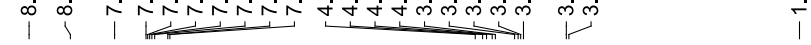

1H NMR $(400 \mathrm{MH}, \mathrm{CDCl})$

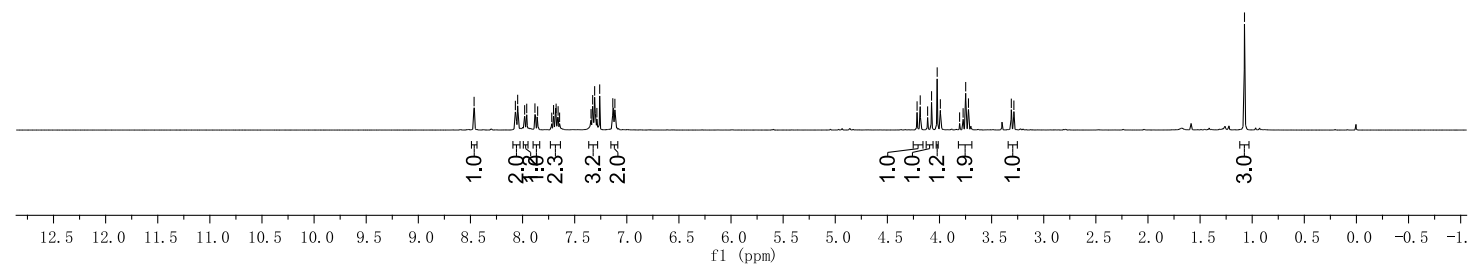



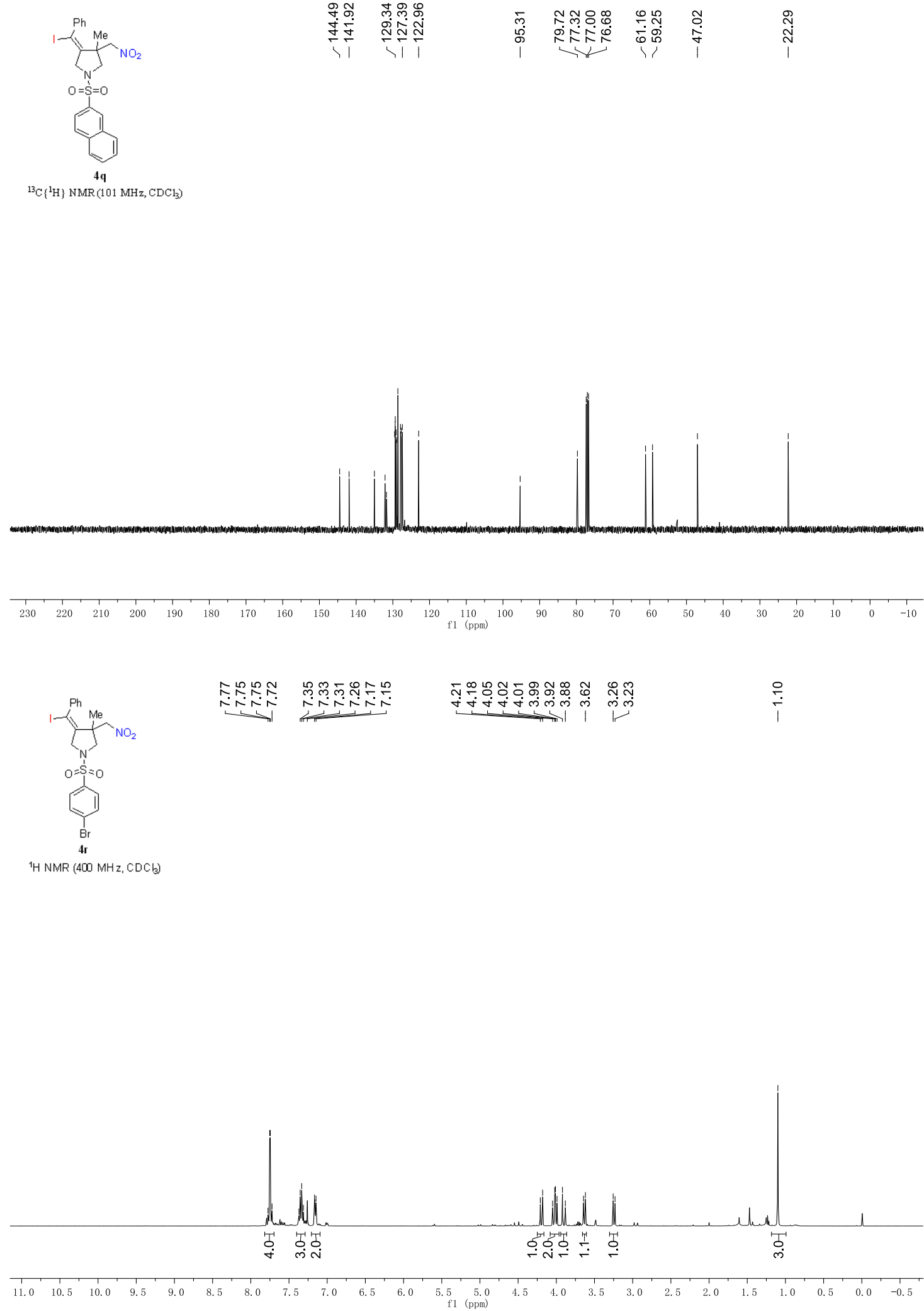


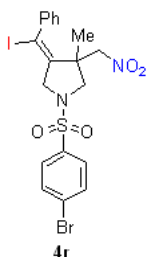

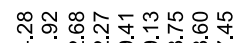

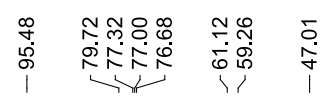

ลั.

${ }^{13} \mathrm{C}\left\{{ }^{1} \mathrm{H}\right\} \operatorname{NMR}\left(101 \mathrm{MHz}, \mathrm{CDCl}_{3}\right)$
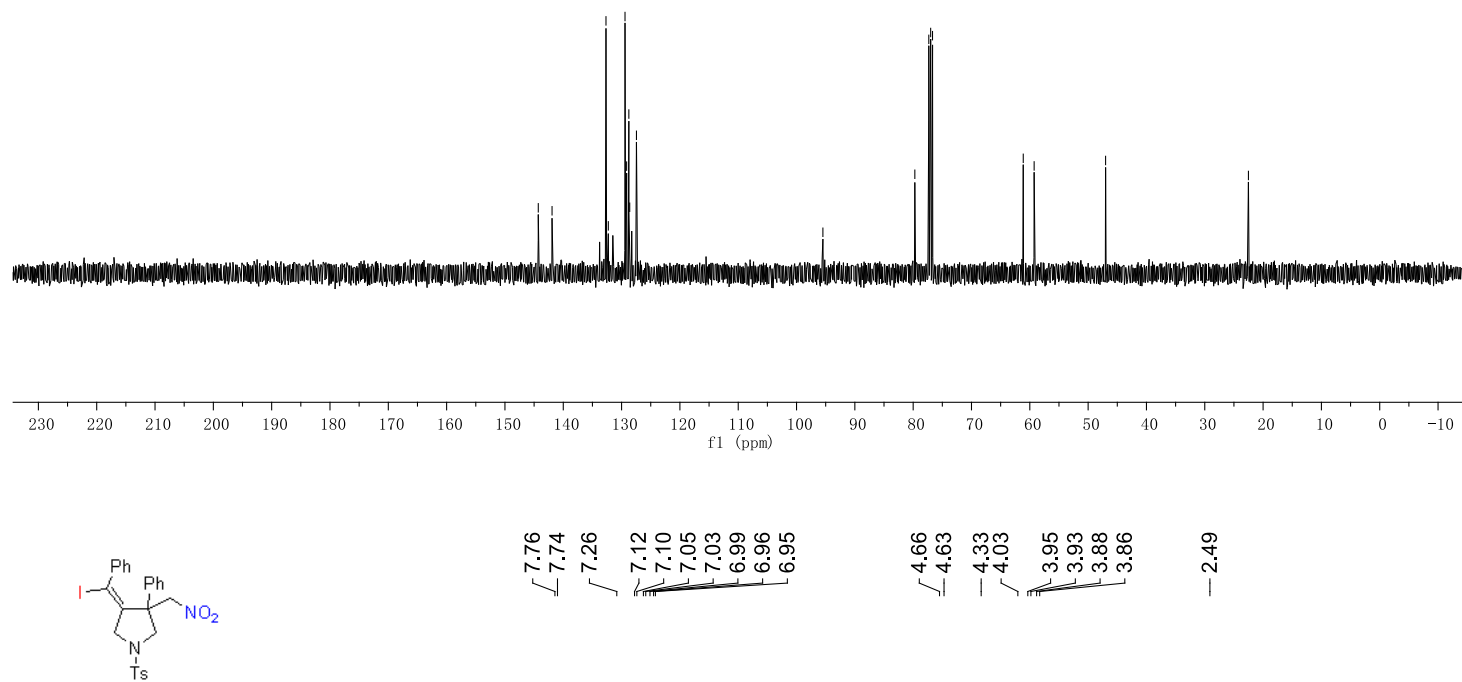

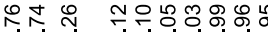

रrivingog

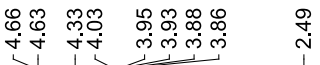

H NMR (400 MHz, $\mathrm{CDCb})$

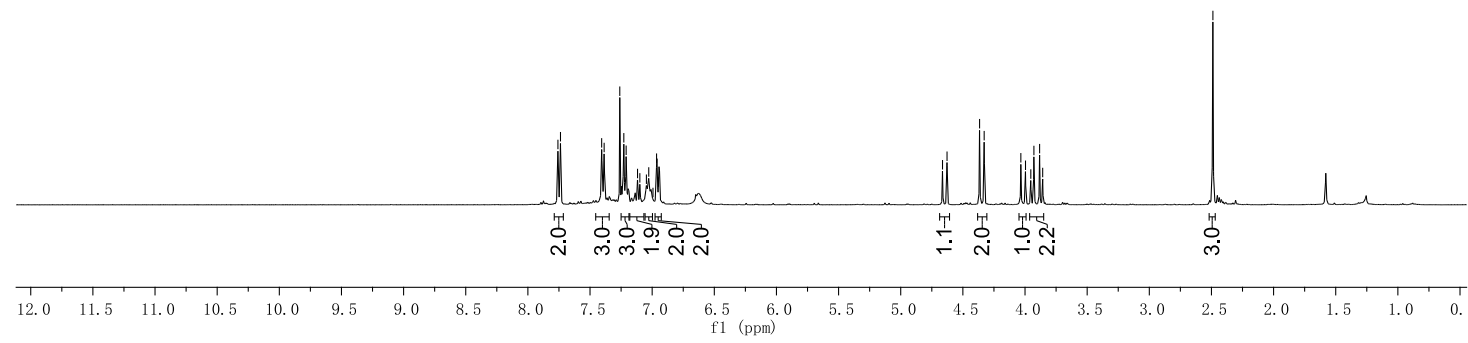




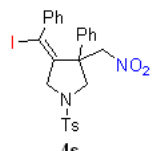

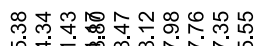

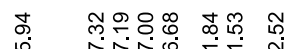

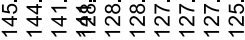

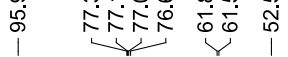

$\stackrel{\stackrel{\leftrightarrow}{+}}{+}$

4 s

${ }^{13} \mathrm{C}\left\{{ }^{1} \mathrm{H}\right\} \operatorname{NMR}\left(101 \mathrm{MHz}, \mathrm{CDCl}_{3}\right)$

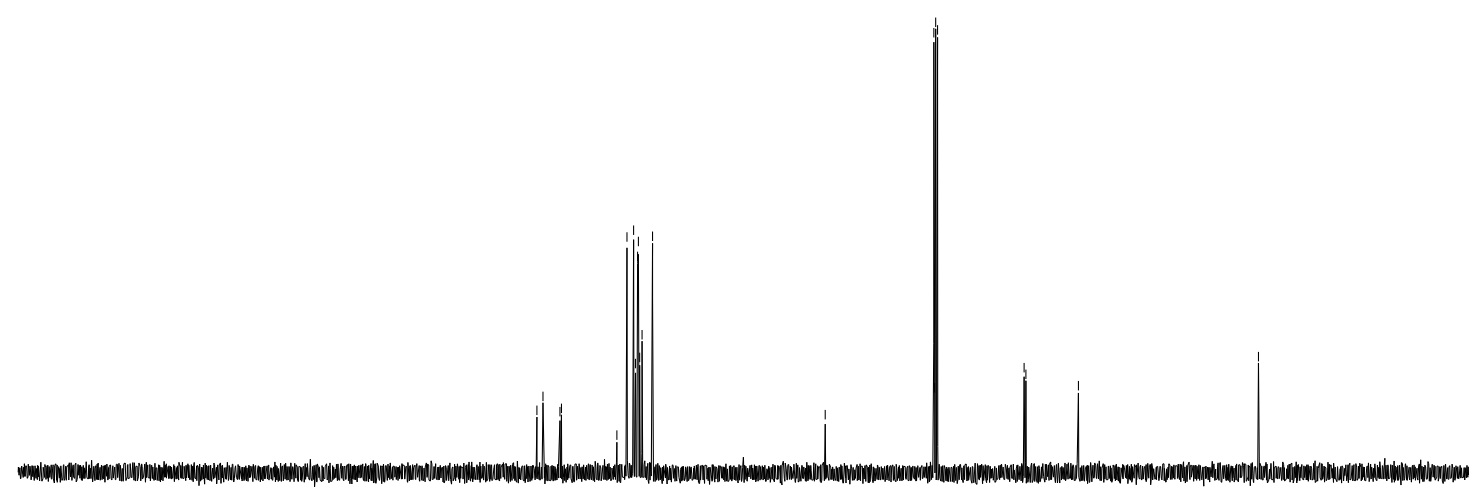

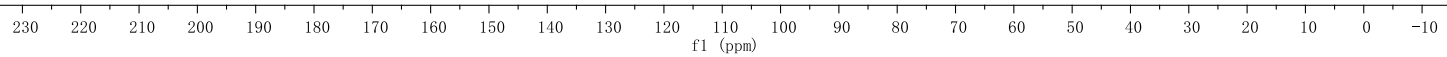

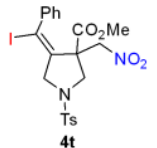

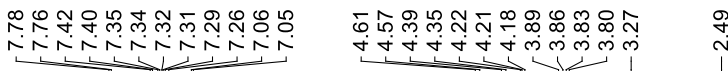

'H NMR $\left(400 \mathrm{MHz}, \mathrm{CDCl}_{3}\right)$

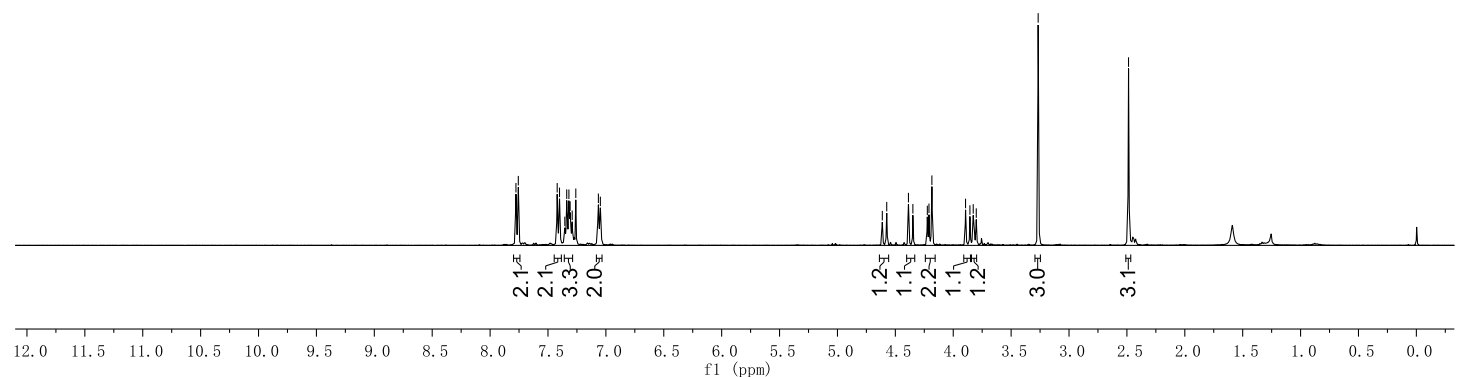




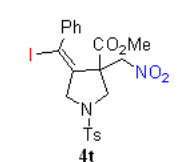

${ }^{13} \mathrm{C}\left\{{ }^{1} \mathrm{H}\right\} \operatorname{NMR}\left(101 \mathrm{MHz}, \mathrm{CDCl}_{3}\right)$
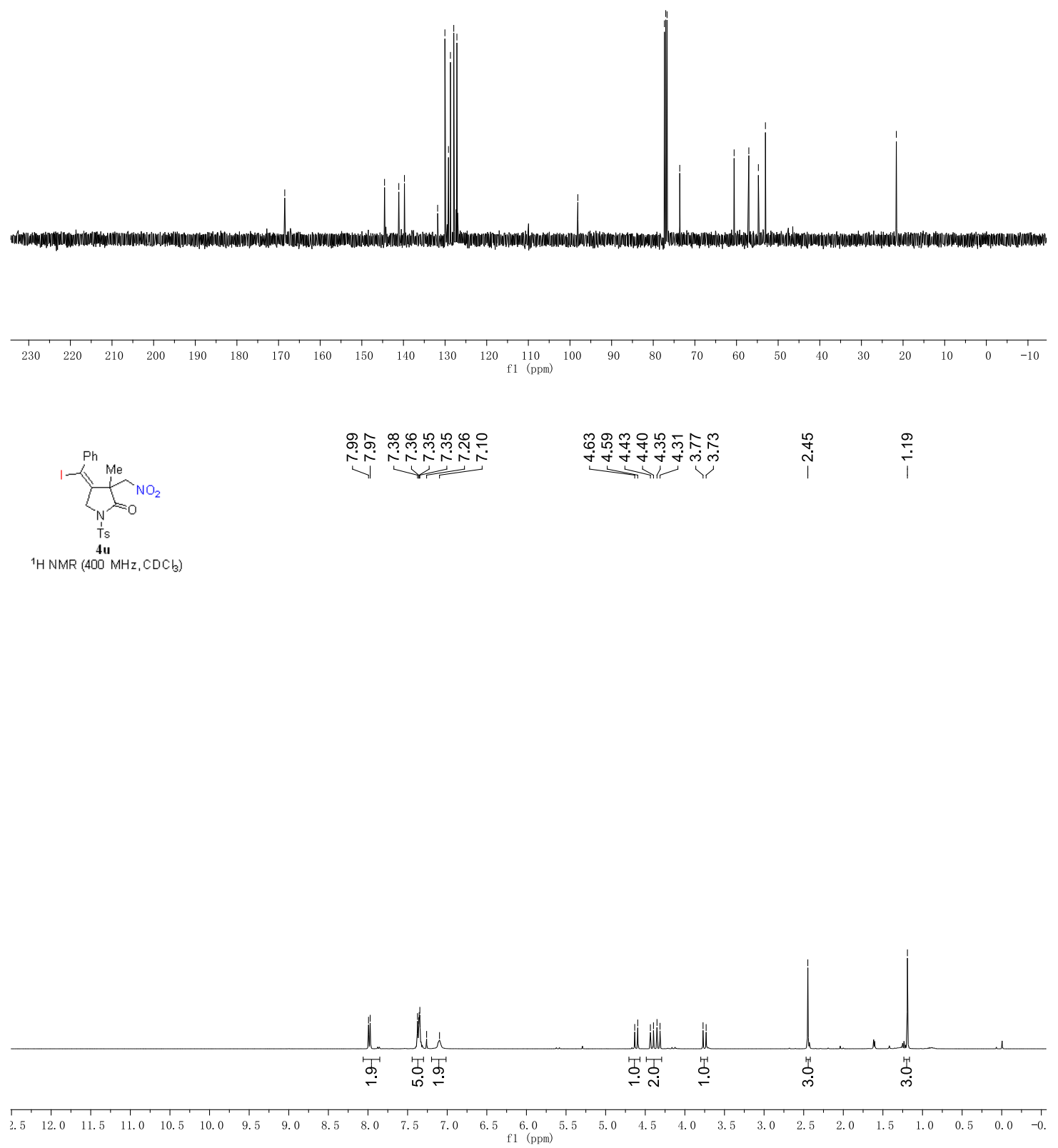


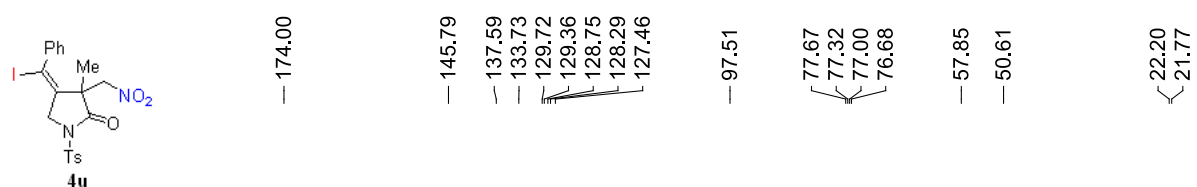

${ }^{13} \mathrm{C}\left\{{ }^{1} \mathrm{H}\right\} \operatorname{NMR}\left(101 \mathrm{MHz}^{\left.-\mathrm{CDCl}_{3}\right)}\right.$
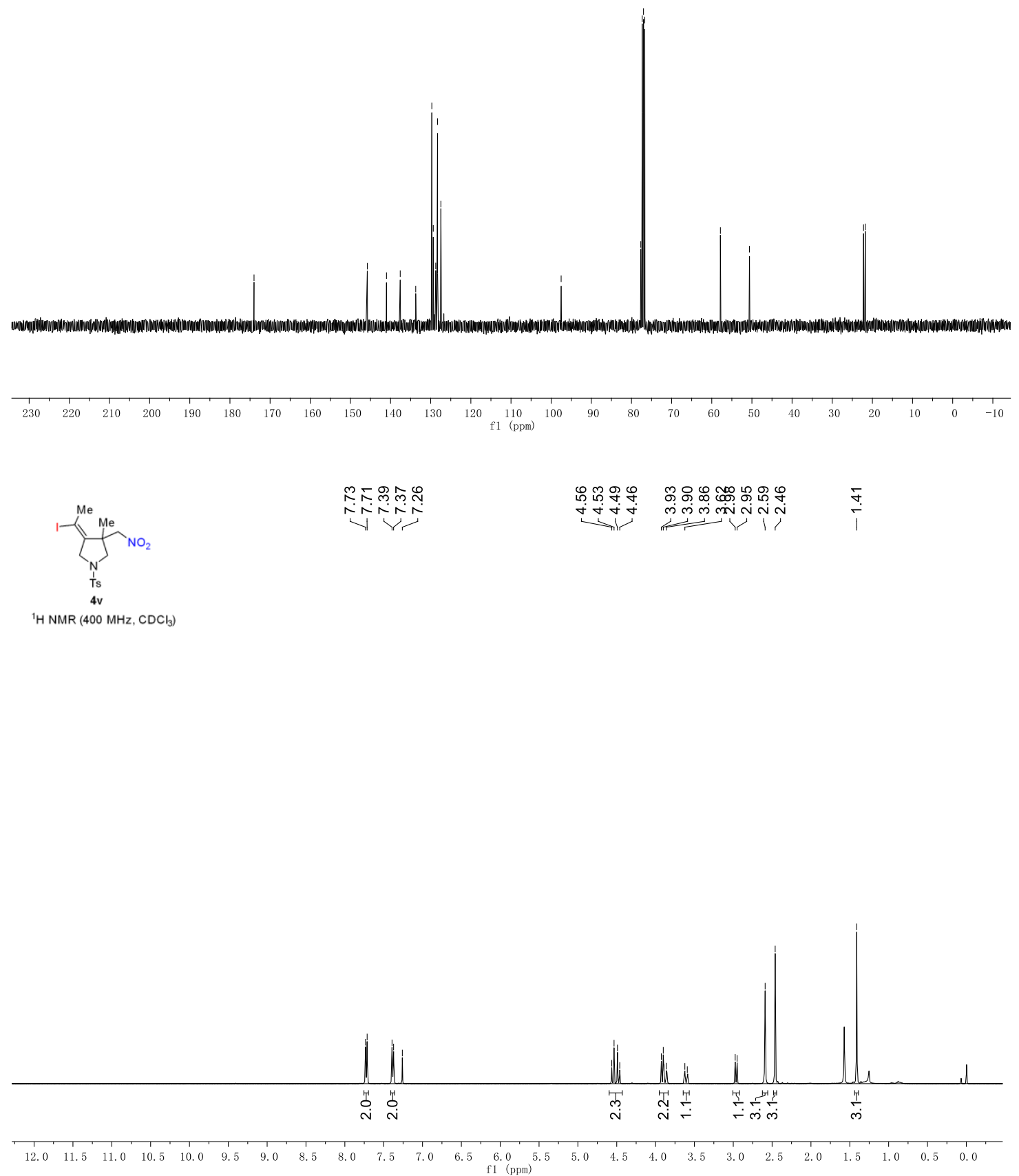


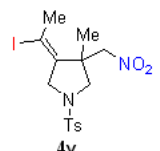

융ำ ำㅇำ
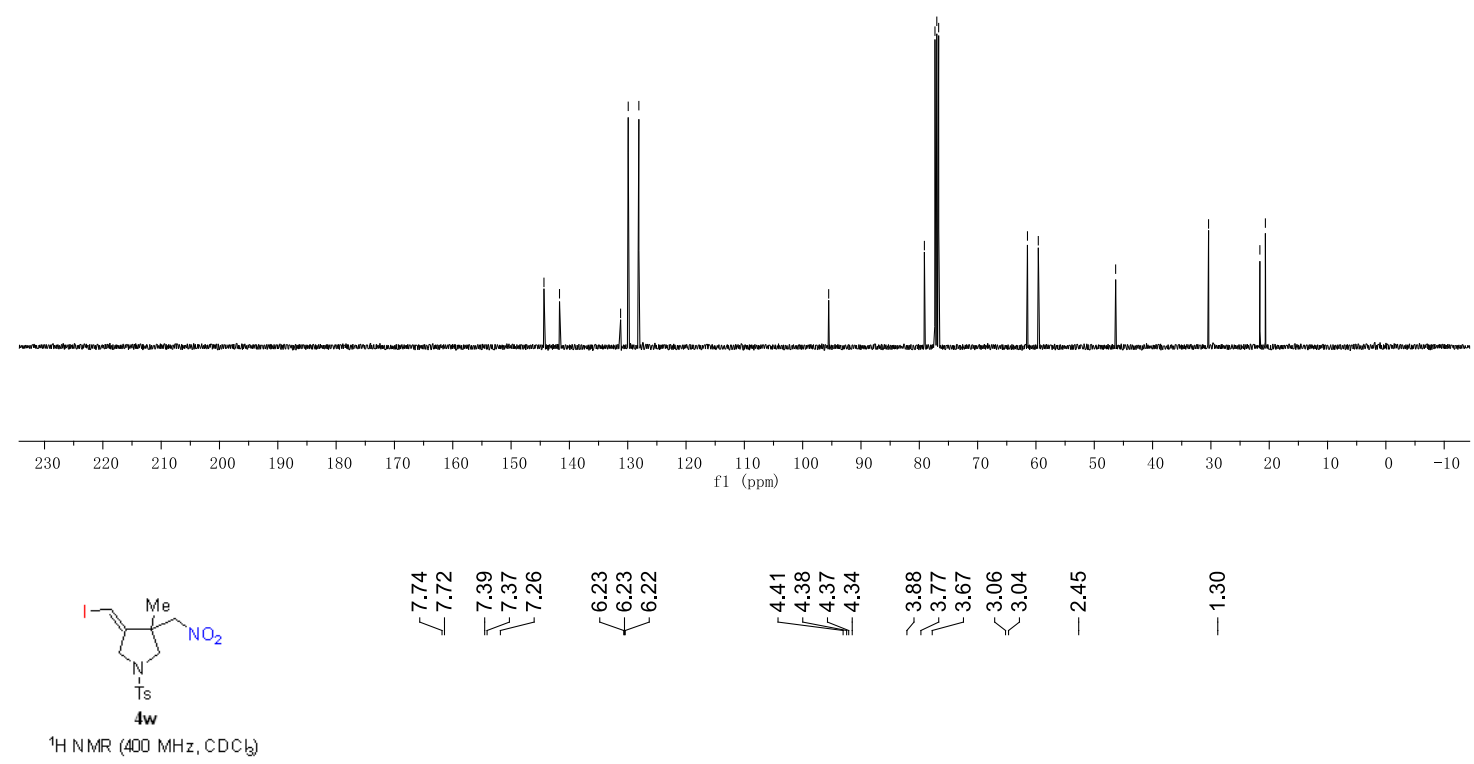

许

${ }^{1} \mathrm{H} N \mathrm{MR}(400 \mathrm{MHz}, \mathrm{CDCl})$

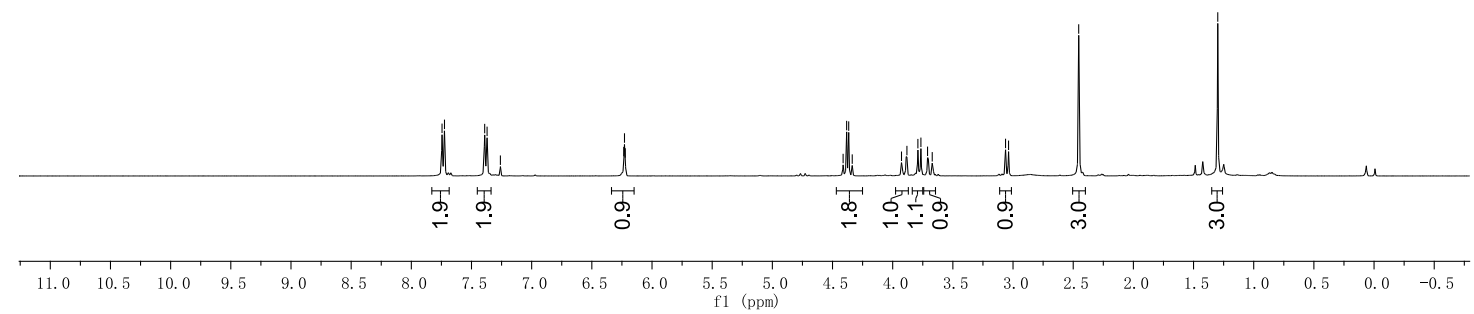




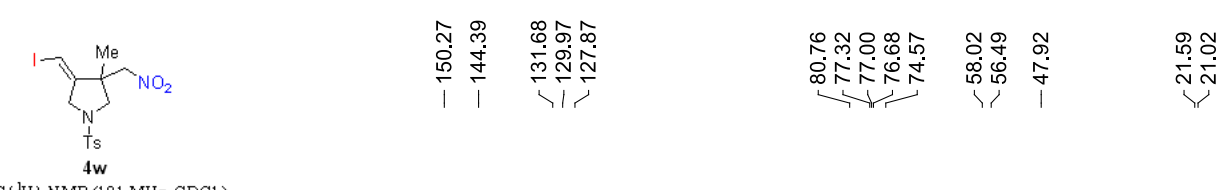

${ }^{13} \mathrm{C}\left\{{ }^{1} \mathrm{H}\right\} \operatorname{NMR}\left(101 \mathrm{MHz}, \mathrm{CDCl}_{3}\right)$
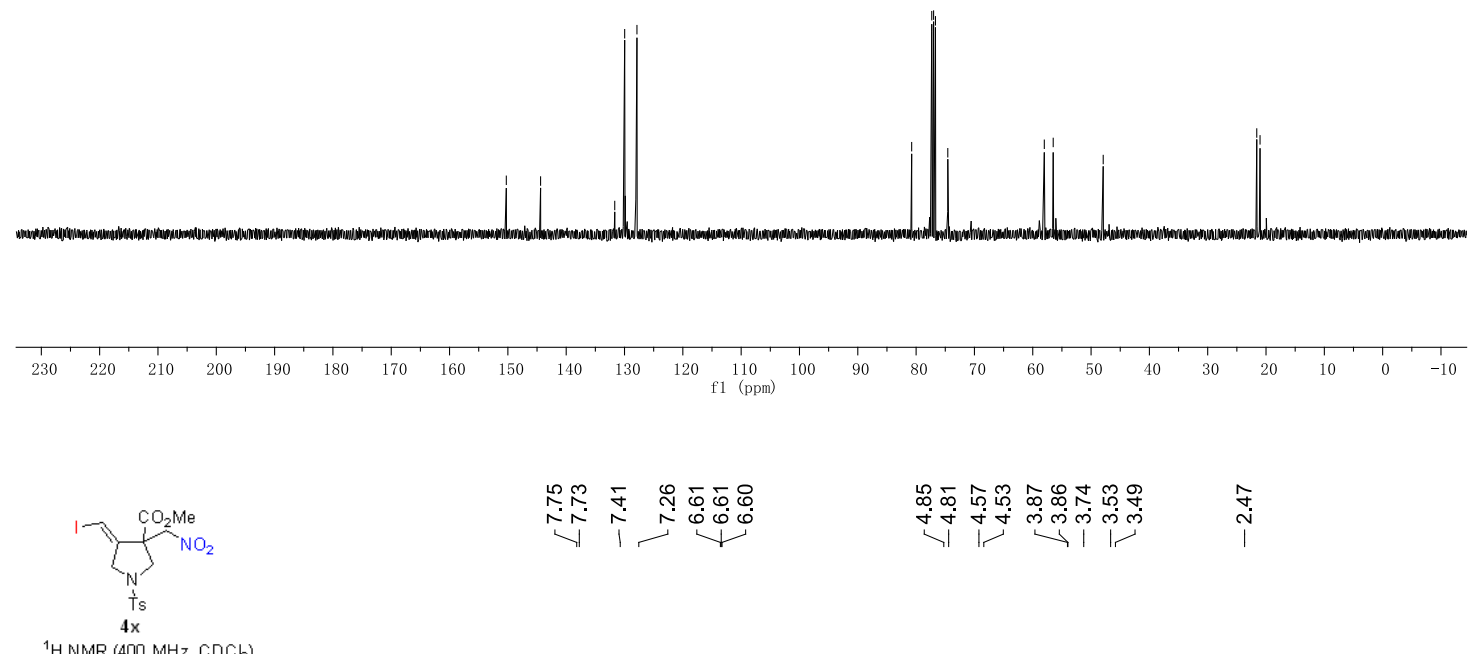

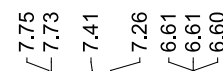

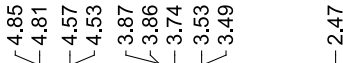

${ }^{1} \mathrm{HNMR}(400 \mathrm{MHz}, \mathrm{CDCb})$

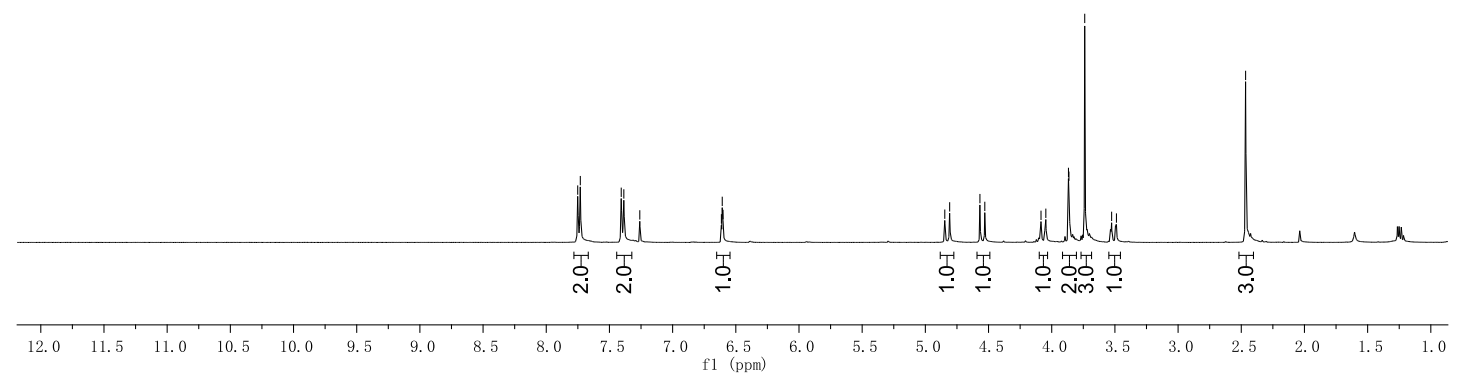



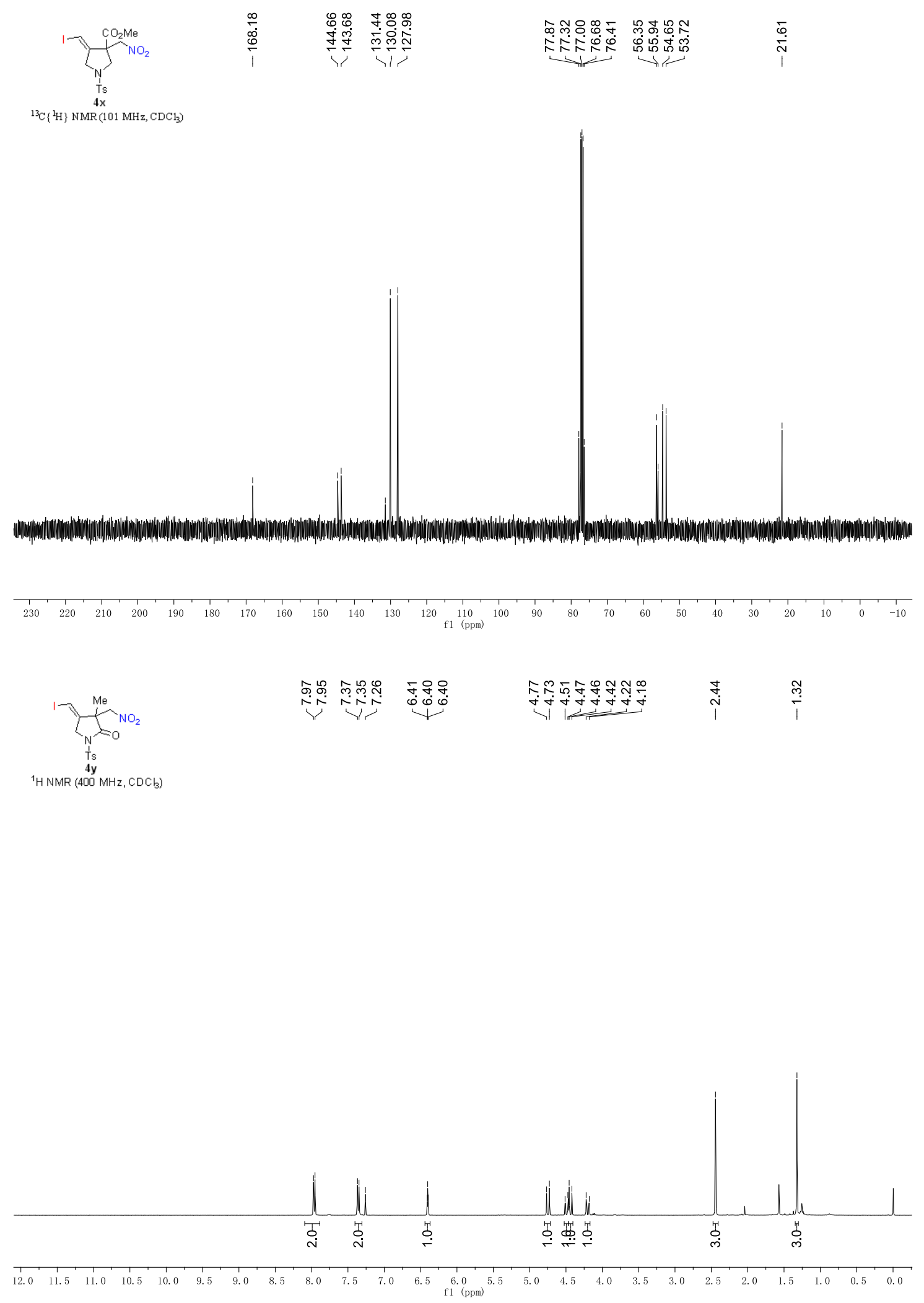


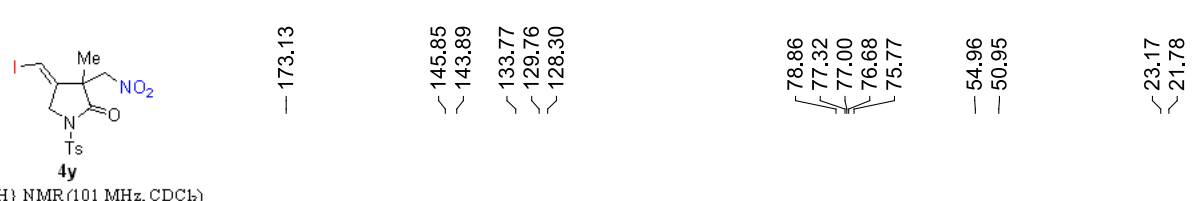

${ }^{13} \mathrm{C}\left\{{ }^{1} \mathrm{H}\right\} \operatorname{MMR}(101 \mathrm{MHz}, \mathrm{CDCl})$
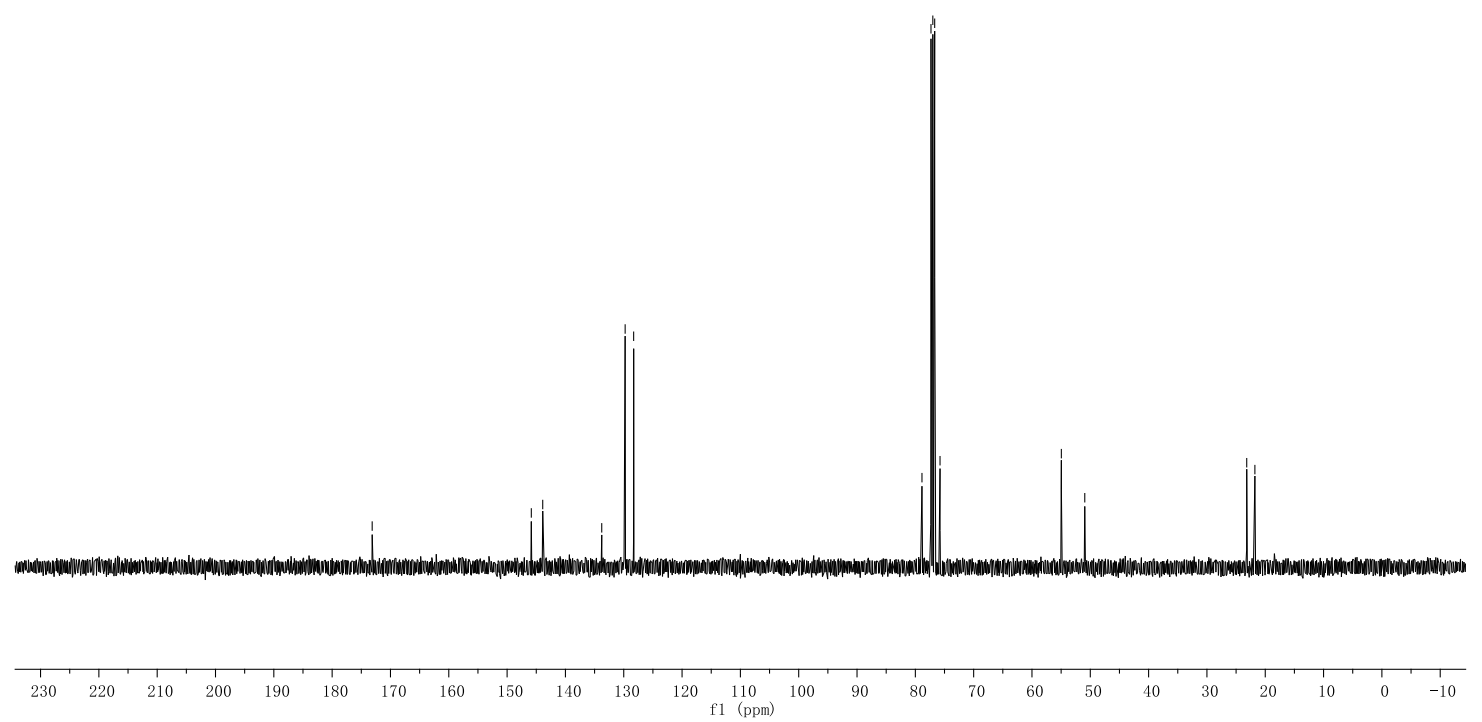

$\sum_{\substack{N \\ T_{5} \\ 5 a}}^{M e} N_{N_{2}}$

${ }^{1}$ H NMR (400 MHz, DMSO-d $)$

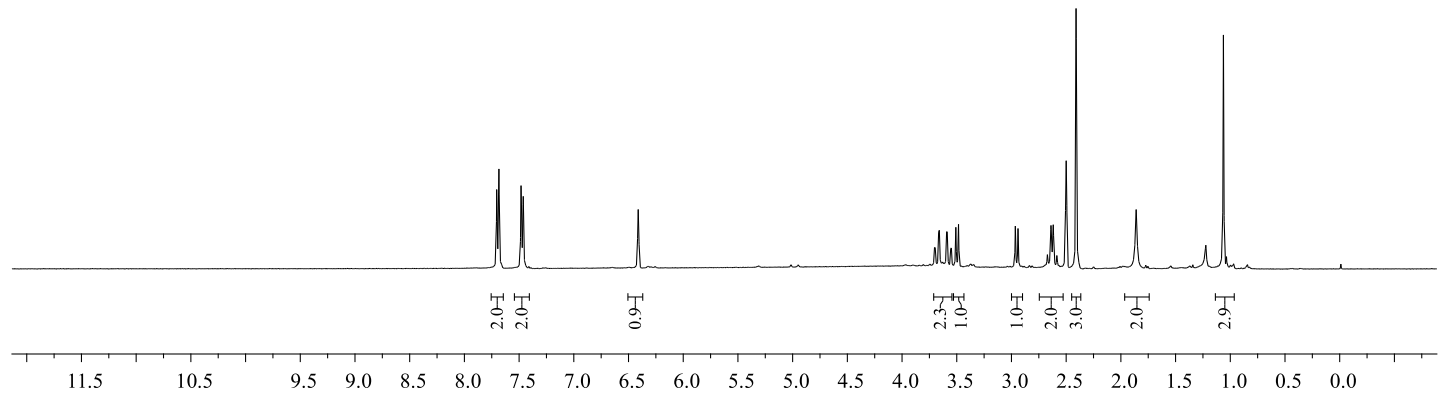




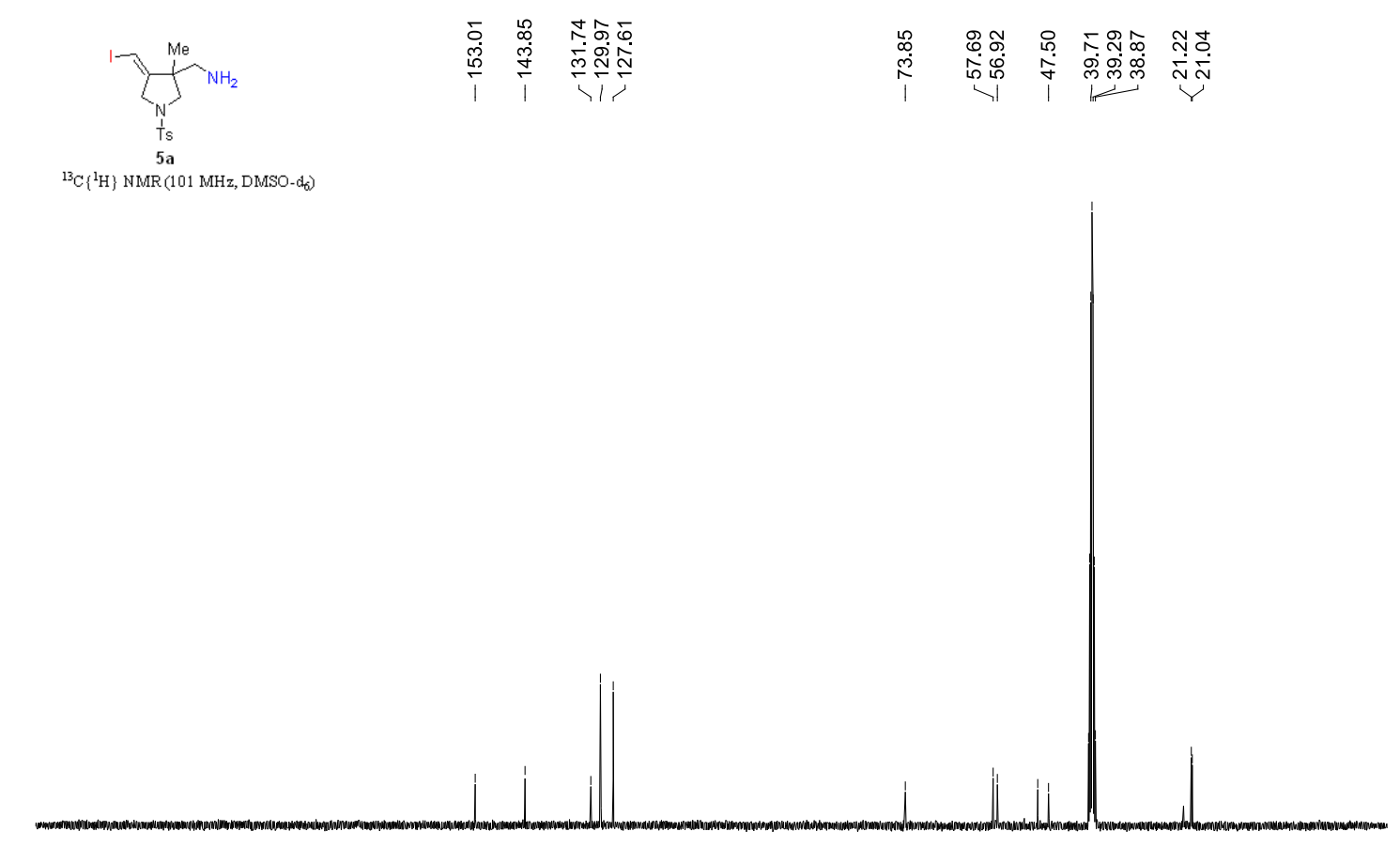

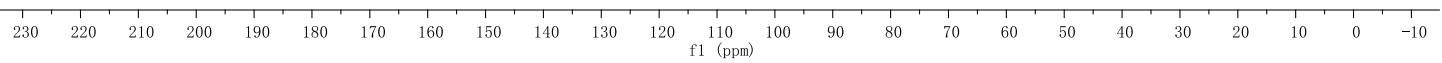

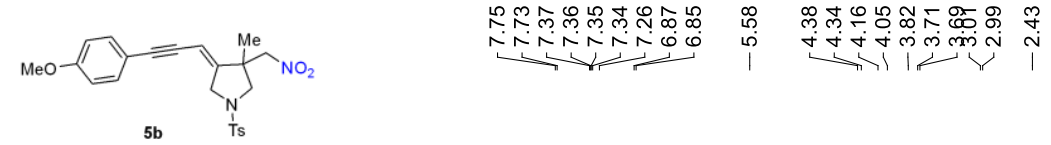

${ }^{1} \mathrm{HNMR}\left(400 \mathrm{MHz}, \mathrm{CDCl}_{3}\right)$

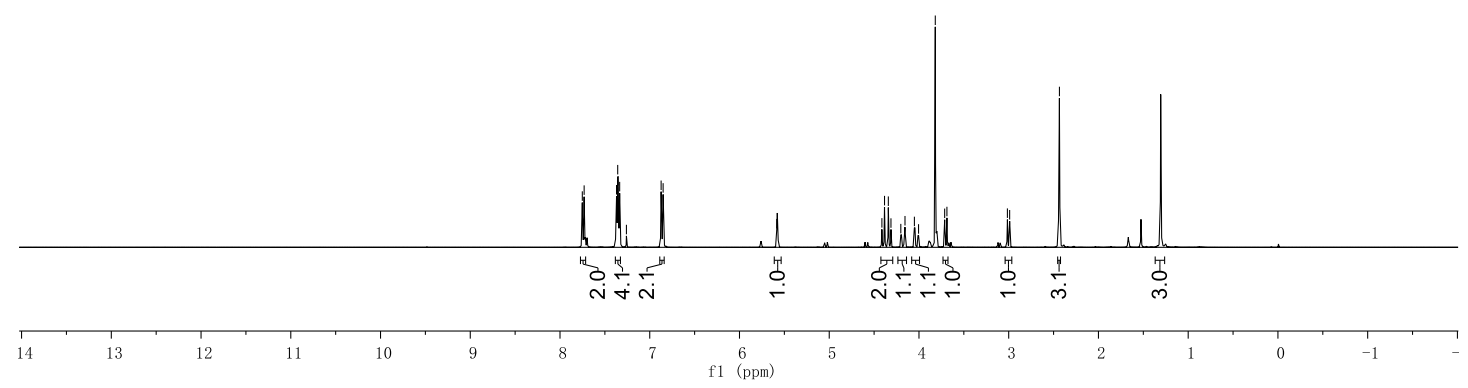




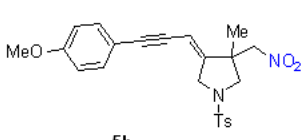

${ }^{13} \mathrm{C}\left\{{ }^{1} \mathrm{H}\right\} \mathrm{NMR}\left(101 \mathrm{MHz}, \mathrm{CDCl}_{3}\right)$
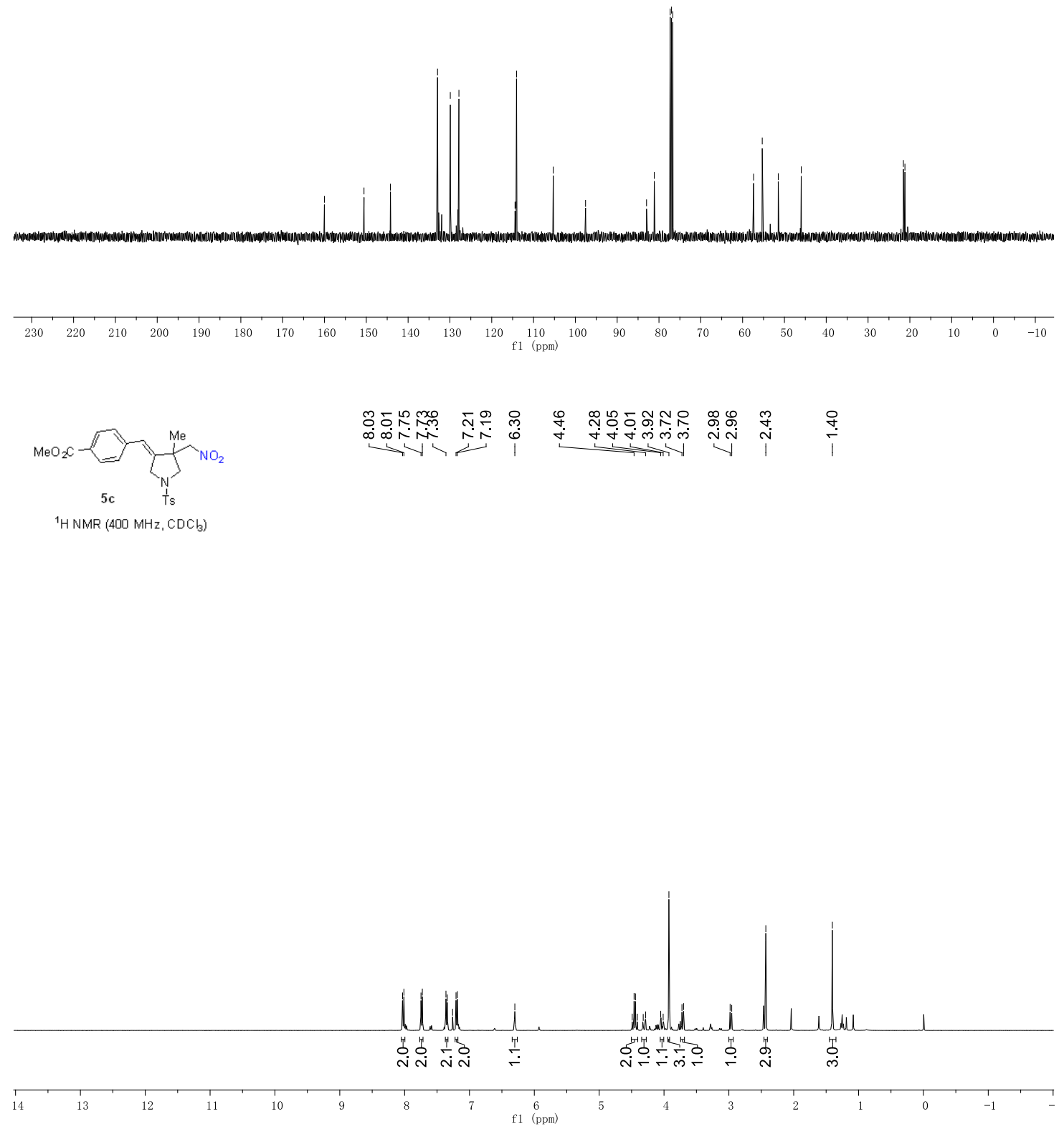


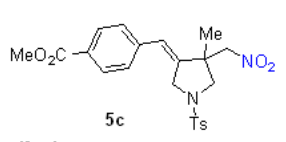

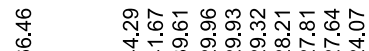

赫过

सำำ

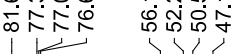

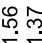

${ }^{13} \mathrm{C}\left\{{ }^{1} \mathrm{H}\right\} \operatorname{NMR}\left(101 \mathrm{MHz}, \mathrm{CDCl}_{3}\right)$

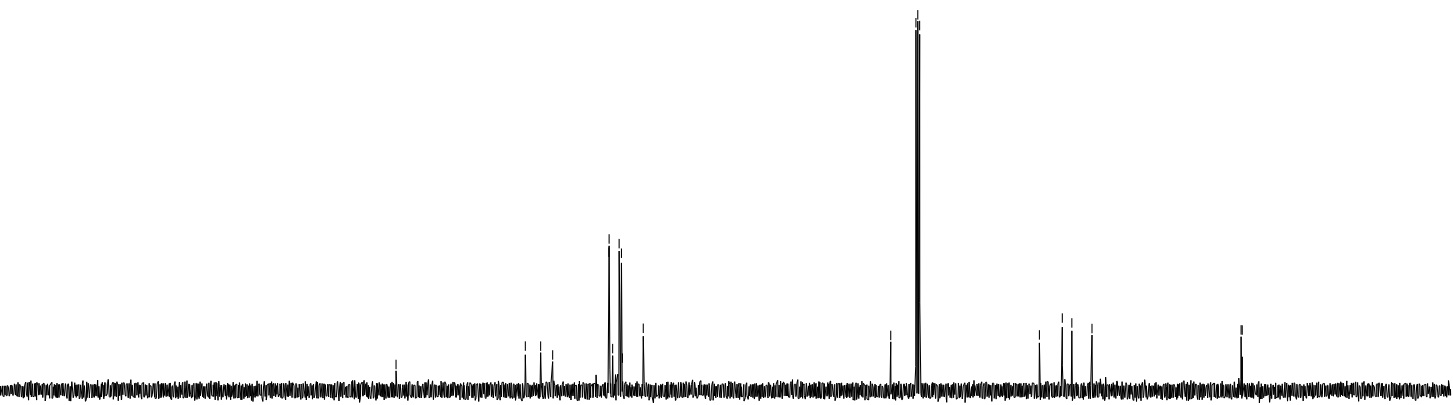

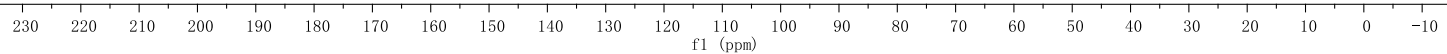

